\title{
Nonequilibrium Statistical Mechanics of Weakly Stochastically Perturbed System of Oscillators
}

\author{
Andrey Dymov
}

\begin{abstract}
We consider a finite region of a $d$-dimensional lattice, $d \in \mathbb{N}$, of weakly coupled harmonic oscillators. The coupling is provided by a nearest-neighbour potential (harmonic or not) of size $\varepsilon$. Each oscillator weakly interacts by force of order $\varepsilon$ with its own stochastic Langevin thermostat of arbitrary positive temperature. We investigate limiting as $\varepsilon \rightarrow 0$ behaviour of solutions of the system and of the local energy of oscillators on long-time intervals of order $\varepsilon^{-1}$ and in a stationary regime. We show that it is governed by an effective equation which is a dissipative SDE with nondegenerate diffusion. Next, we assume that the interaction potential is of size $\varepsilon \lambda$, where $\lambda$ is another small parameter, independent from $\varepsilon$. Solutions corresponding to this scaling describe small low temperature oscillations. We prove that in a stationary regime, under the limit $\varepsilon \rightarrow 0$, the main order in $\lambda$ of the averaged Hamiltonian energy flow is proportional to the gradient of temperature. We show that the coefficient of proportionality, which we call the conductivity, admits a representation through stationary space-time correlations of the energy flow. Most of the results and convergences we obtain are uniform with respect to the number of oscillators in the system.
\end{abstract}

\section{Contents}

1. Introduction 1826

1.1. Motivation and Setup 1826

1.2. Main Results: The Limiting Dynamics 1829

1.3. Main Results: The Energy Transport 1832

1.4. Assumptions and Agreements 1834

2. The Limiting Dynamics 1835

2.1. $\quad$ Estimates on Solutions 1835

2.2. $a$-Variables and the Effective Equation 1840 
2.3. Averaging Theorems

2.4. The Limiting Behaviour of the Local Energy 1845

2.5. Rotation Invariance of the Effective Equation 1846

3. The Energy Transport 1847

3.1. The Limiting Behaviour of the Energy Flow 1847

$\begin{array}{ll}\text { 3.2. Examples } & 1855\end{array}$

3.3. Space-Time Correlations of the Energy Flow 1857

4. Proofs of the Averaging Theorems 1860

4.1. Averaging Lemma 1860

4.2. Proof of Theorem 2.4 1863

$\begin{array}{ll}\text { 4.3. Proof of Theorem 2.8 } & 1867\end{array}$

4.4. The $\mathcal{C}_{\infty}$-Effective Equation 1871

$\begin{array}{ll}\text { Acknowledgements } & 1874\end{array}$

$\begin{array}{lll}\text { Appendix A. Uniformity of Mixing } & 1874\end{array}$

$\begin{array}{ll}\text { Appendix B. Low Temperature Regime } & 1878\end{array}$

$\begin{array}{lll}\text { Appendix C. Resonant Averaging } & 1879\end{array}$

$\begin{array}{ll}\text { References } & 1879\end{array}$

\section{Introduction}

\subsection{Motivation and Setup}

Investigation of the energy transport in crystals is one of the central problems in nonequilibrium statistical mechanics. In particular, a derivation of the Fourier law and the Green-Kubo formula from the microscopic dynamics of particles is of great interest (see $[12,36]$ ). In the classical setting, one investigates the energy transport in a Hamiltonian system, interacting with thermal baths of different temperatures. This interaction is weak in geometrical sense: the thermal baths are coupled with the Hamiltonian system only through a boundary of the latter. Unfortunately, for the moment of writing this problem seems to be out of reach, due to the weakness of the interaction. In this setting, even the existence of a stationary state in the system is not obvious (see $[13-15,20,21,24,38]) .{ }^{1}$ That is why usually one modifies the system to get additional ergodic properties. Standard ways to achieve that are (1) to consider a weak perturbation of the hyperbolic system of independent particles $[16,40] ;(2)$ to perturb each mode of the Hamiltonian system by sufficiently strong noise $[2,6-8,10,11,33]$. Following the second way, usually one perturbs the Hamiltonian dynamics by very special noise, preserving the energy of the system $[2,6-8,33]$, or one couples each particle of the Hamiltonian system with its own stochastic Langevin-type thermostat $[6,10,11]$. In the latter case, the stochastic perturbation has rather natural structure; however, the energy of the system is not conserved.

Let us discuss some of results mentioned above in more details. In [7], the authors study a chain of harmonic oscillators, perturbed by energy preserving

\footnotetext{
${ }^{1}$ See also $[18,43]$ for a similar problem in deterministic setting.
} 
stochastic exchange of momentum between neighbouring oscillators. Coupling the first and the last oscillator to thermal baths of different temperatures $\mathcal{T}_{1}$ and $\mathcal{T}_{2}$, they prove the Fourier law, i.e. that the stationary averaged flow of energy is approximately proportional to $\left(\mathcal{T}_{1}-\mathcal{T}_{2}\right) / N$ for a large number of particles $N$ and a small difference $\mathcal{T}_{1}-\mathcal{T}_{2}$. In [2], the authors consider a system of oscillators, where each oscillator is perturbed by a stochastic dynamics, conserving momentum and energy. They study existence of the Green-Kubo conductivity. In [10], the authors consider a system of harmonic oscillators, where each oscillator is coupled with a stochastic Langevin thermostat. Fixing temperatures of the "exterior" left and right thermostats they find and study the self-consistent temperature profile for the "interior" thermostats, i.e. a temperature profile such that in a stationary regime there is no average flow of energy between the "interior" thermostats and the system of oscillators. Then, they prove that with respect to this temperature profile, the Fourier law and the Green-Kubo formula hold.

In all the works listed above, the stochastic perturbation is of order one. It is natural to study the case when it goes to zero. Such situation was investigated in [3-5]. In [3], the authors study the FPU-chain, where the nonlinearity is replaced by an energy preserving stochastic exchange of momentum between neighbouring nodes. They investigate the energy transport under the limit when the strength of this exchange tends to zero. In [4], the authors consider a chain of harmonic oscillators, where each oscillator is weakly perturbed by energy preserving noise of size $\varepsilon$ and an anharmonic potential of size $\nu$, $\nu \leq \varepsilon$. They investigate limiting as $\varepsilon \rightarrow 0$ behaviour of an upper bound for the Green-Kubo conductivity. In [5], the authors study an infinite chain of cells weakly coupled by a potential of size $\nu$, where each cell is weakly perturbed by energy preserving noise of size $\varepsilon$. Expanding the Green-Kubo conductivity in a formal series $\kappa(\nu, \varepsilon)=\sum_{n \geq 2} \nu^{n} \kappa_{n}(\varepsilon)$, they find the term $\kappa_{2}(\varepsilon)$ and investigate its limiting behaviour as $\varepsilon \rightarrow 0$. It is argued that under the formal limit $\nu \rightarrow 0$ the Fourier law holds with the conductivity $\kappa_{2}(\varepsilon)$.

In the present work, we consider a system of oscillators, situated in nodes of a bounded set $\mathcal{C} \subset \mathbb{Z}^{d}, d \in \mathbb{N}$, and interacting via a nearest-neighbour type potential. The system is given by the Hamiltonian

$$
H^{\nu}(p, q)=\sum_{j \in \mathcal{C}}\left(\frac{p_{j}^{2}}{2}+U_{j}\left(q_{j}\right)\right)+\frac{\nu}{2} \sum_{j, k \in \mathcal{C}:|j-k|=1} V\left(q_{j}, q_{k}\right)
$$

where $(p, q)=\left(p_{j}, q_{j}\right)_{j \in \mathcal{C}} \in \mathbb{R}^{2|\mathcal{C}|}, \nu>0$ and for $j \in \mathbb{Z}^{d}$ by $|j|$ we denote the $l_{1}$-norm. The interaction potential $V$ satisfies $V(x, y) \equiv V(y, x)$, is $C^{2}$-smooth and has at most a polynomial growth at infinity (for precise assumptions see Sect. 1.4). The pinning potentials $U_{j}$ will be specified later. Following the second strategy above, we couple each oscillator with its own stochastic Langevin thermostat of arbitrary temperature $\mathcal{T}_{j}$ by coupling of size $\varepsilon$, where the temperatures satisfy

$$
0<\mathcal{T}_{j}<C<\infty
$$


with a constant $C$ independent from $j$. The resulting system obeys the equation

$$
\begin{aligned}
& \frac{\mathrm{d}}{\mathrm{d} t} q_{j}=p_{j}, \\
& \frac{\mathrm{d}}{\mathrm{d} t} p_{j}=-\partial_{q_{j}} U_{j}\left(q_{j}\right)-\nu \sum_{k:|k-j|=1} \partial_{q_{j}} V\left(q_{j}, q_{k}\right)-\varepsilon p_{j}+\sqrt{2 \varepsilon \mathcal{T}_{j}} \frac{\mathrm{d}}{\mathrm{d} t} \beta_{j}, \quad j \in \mathcal{C},
\end{aligned}
$$

where $\beta=\left(\beta_{j}\right)_{j \in \mathcal{C}} \in \mathbb{R}^{|\mathcal{C}|}$ is a standard $|\mathcal{C}|$-dimensional Brownian motion. Our goal is to investigate limiting as $\varepsilon \rightarrow 0$ behaviour of solutions and of energy transport for Eq. (1.3) on long time intervals and in a stationary regime. So, as in the classical setting given above, we are interested in the case of weak interaction between the Hamiltonian system and the thermal baths; however, the weakness is understood in a different, non-geometrical sense. Unfortunately, we will have to assume that the coupling constant $\nu$ goes to zero simultaneously with $\varepsilon$, with some precise scaling which will be discussed later.

Our system admits a clear physical interpretation, so not only it can be considered as a toy model for a purely Hamiltonian situation, but it is of independent interest. Indeed, since the stochastic perturbation is provided by the weak coupling with the Langevin thermostats, one can think about the transport of energy through a crystal plugged in some medium of a given temperature distribution and weakly interacting with it. However, since the energy of the system is not conserved, in our case non-Hamiltonian effects seem to be stronger than in the systems above, perturbed by vanishing energy conserving noises. To have some kind of energy preservation, one could consider the self-consistent temperature profile, if the latter exists.

Since the systems of statistical physics are of very large dimension, it is crucial to control the dependence of the systems on their size. Let us emphasize that most of results and convergences we obtain are uniform with respect to the choice of the set $\mathcal{C}$ (in particular, with respect to its size), so they satisfy this physical requirement.

We started our study in [19]. There we considered a system of rotators, where the uncoupled rotators were assumed nonlinear, while the coupling constant $\nu$ was chosen as $\nu=\varepsilon^{a}, a \geq 1 / 2$. In the case $1 / 2 \leq a<1$, the rotators were required to have alternated spins (in such a way we excluded from the system too strong resonances). Using the averaging method of Khas'minski-Freidlin-Wentzell type in the form developed in [30], we found an autonomous (stochastic) equation for the local energy which described its limiting (as $\varepsilon \rightarrow 0$ ) behaviour on long time intervals of order $\varepsilon^{-1}$ and in a stationary regime, uniformly with respect to the choice of the set $\mathcal{C}$. However, it turned out that this equation did not feel the Hamiltonian interaction of rotators, so that in the considered system there was no Hamiltonian energy flow under the limit $\varepsilon \rightarrow 0$, neither on time intervals of order $\varepsilon^{-1}$ nor in the stationary regime. The reason is that under natural assumptions resonances of the system of uncoupled nonlinear rotators have Lebesgue measure zero. A weak coupling does not change the situation significantly, so that the noise pushes out rapidly the perturbed dynamics to nonresonant area, where the 
Hamiltonian terms average out. In $[22],{ }^{2}$ similar results were obtained for a system similar to (1.3), where the uncoupled oscillators were nonlinear and $\nu=\varepsilon$ (however, no information was provided about dependence of the results on the choice of the set $\mathcal{C}$ and behaviour of the system in a stationary regime).

To observe the Hamiltonian energy flow, in the present paper we study the situation when the uncoupled oscillators are linear and have the same frequencies, so that

$$
U_{j}\left(q_{j}\right)=\frac{q_{j}^{2}}{2} \quad \text { for all } j \in \mathcal{C} .
$$

In this case, Eq. (1.3) is strongly resonant, see below. We choose the coupling constant $\nu$ as:

$$
\nu=\lambda \varepsilon, \text { where } 0<\lambda \leq 1,
$$

since we are not able to treat the case when $\varepsilon=o(\nu)$, while the choice (1.5) already provides nontrivial results. In the first part of the paper, devoted to the study of the limiting as $\varepsilon \rightarrow 0$ behaviour of solutions of Eqs. (1.3)-(1.5), we assume that $\lambda$ is a fixed number satisfying $0<\lambda \leq 1$. In the second part, treating the limiting (as $\varepsilon \rightarrow 0$ ) behaviour of the energy flow, we assume that $\lambda$ is a small parameter, independent from $\varepsilon$ (but usually also fixed). Equations (1.3)-(1.5) with $\lambda \ll 1$ describe small amplitude low temperature oscillations, see Appendix B for explanation.

Since $\lambda$ will be mostly fixed while $\varepsilon$ will go to zero, natural time scale for Eqs. (1.3)-(1.5) is $t \sim \varepsilon^{-1}$. It is convenient to introduce a slow time $\tau=\varepsilon t$, so that $\tau \sim 1$. Then, Eqs. (1.3)-(1.5) take the form

$$
\dot{q}_{j}=\varepsilon^{-1} p_{j}, \quad \dot{p}_{j}=-\varepsilon^{-1} q_{j}-\lambda \sum_{k:|k-j|=1} \partial_{q_{j}} V\left(q_{j}, q_{k}\right)-p_{j}+\sqrt{2 \mathcal{T}_{j}} \dot{\beta}_{j}, \quad j \in \mathcal{C},
$$

where the dot denotes the derivative with respect to $\tau$. It is well known that Eq. (1.6) has a unique solution, defined for all $\tau \geq 0$ (see [26]), and is mixing ${ }^{3}$ (see Appendix A).

\subsection{Main Results: The Limiting Dynamics}

Our first goal is to investigate the limiting as $\varepsilon \rightarrow 0$ dynamics of Eq. (1.6) for fixed $\lambda$ satisfying $0<\lambda \leq 1$. Introduce the action-angle variables for the system of uncoupled oscillators, given by the Hamiltonian $H^{0}$,

$$
\begin{aligned}
I & =\left(I_{j}\right)_{j \in \mathcal{C}} \in \mathbb{R}^{|\mathcal{C}|}, \quad I_{j}:=\frac{p_{j}^{2}+q_{j}^{2}}{2} \\
\text { and } \quad \varphi & =\left(\varphi_{j}\right)_{j \in \mathcal{C}} \in \mathbb{T}^{|\mathcal{C}|}, \quad \varphi_{j}:=\arg \left(p_{j}+i q_{j}\right),
\end{aligned}
$$

where $i$ denotes the imaginary unit and we put $\arg 0:=0$. Then, Eq. (1.6) takes the form

$$
\dot{I}_{j}=\ldots, \quad \dot{\varphi}_{j}=\varepsilon^{-1}+\cdots, \quad j \in \mathcal{C},
$$

\footnotetext{
${ }^{2}$ See also Chapter 9.3 in [23].

3 i.e. it has a unique stationary measure and its solutions weakly converge as $\tau \rightarrow \infty$ to this measure in distribution.
} 
where the dots stand for terms of order one (as $\varepsilon \rightarrow 0$ ). Equation (1.8) describes a strongly resonant fast-slow system: the actions change slowly while the dynamics of angles can be decomposed to a fast rotation along the diagonal $\mathbf{1}=(1, \ldots, 1)$ of the torus $\mathbb{T}^{|\mathcal{C}|}$, and a slow drift in $|\mathcal{C}|-1$ ortogonal directions $\xi^{m}, \xi^{m} \cdot \mathbf{1}=0,1 \leq m \leq|\mathcal{C}|-1$. A usual way of studying of the limiting as $\varepsilon \rightarrow 0$ dynamics of such systems is the method of resonant averaging (see [1]). The latter suggests to pass to the variables $(I, \psi, \eta) \in \mathbb{R}^{|\mathcal{C}|} \times \widetilde{\mathbb{T}}^{|\mathcal{C}|}$, where $(\psi, \eta)=\left(\psi_{1} \ldots, \psi_{|\mathcal{C}|-1}, \eta\right) \in \widetilde{\mathbb{T}}^{|\mathcal{C}|}$ are coordinates of the vector of angles $\varphi$ with respect to the basis $\left(\xi^{1}, \ldots, \xi^{|\mathcal{C}|-1}, \mathbf{1}\right)$, and $\widetilde{\mathbb{T}}^{|\mathcal{C}|}$ denotes the torus $\mathbb{T}^{|\mathcal{C}|}$ expanded in a suitable way. Then, Eq. (1.8) takes the form

$$
\dot{I}_{j}=\ldots, \quad \dot{\psi}_{m}=\ldots, \quad \dot{\eta}=\varepsilon^{-1}+\cdots, \quad \text { where } \quad 1 \leq m \leq|\mathcal{C}|-1 .
$$

Equation (1.9) is a fast-slow system with the unique fast variable $\eta$, so the limiting as $\varepsilon \rightarrow 0$ behaviour of the slow variables $(I, \psi)$ is expected to be given by the averaging of $(I, \psi)$-equations with respect to $\eta$, which further on we call the resonant averaging. However, to establish this, we cannot use the usual scheme which suppose to work in the action-angle variables, since writing the $\varphi$-equations in more details we find $\dot{\varphi}_{j}=\varepsilon^{-1}+I_{j}^{-1} \cdot \ldots, j \in \mathcal{C}$, so that they have singularities at the locus $\left\{I=\left(I_{j}\right)_{j \in \mathcal{C}}: I_{j}=0\right.$ for some $\left.j \in \mathcal{C}\right\}$. Moreover, the resonant averaging of the $I$-equations has weak singularities there. To overcome this difficulty, we use a method developed in $[27,28]$ for a non-resonant case and in [29] for a resonant one. It suggests to find and study an effective equation, written in the regular $(p, q)$-variables, which governs the limiting as $\varepsilon \rightarrow 0$ dynamics of the $(I, \psi)$-components of solutions of Eq. (1.6). To this end, in a general situation one should use that the $(I, \psi)$-components of solutions of the effective equation have to satisfy the resonant averaging of the $(I, \psi)$-equations from (1.9). However, following [29], we note that in our case, where the system of uncoupled oscillators is linear, there exists a simple way to find the effective equation without using the $(I, \psi)$-coordinates, so that the singularities do not appear at all. It is based on a passage to a fast-rotating coordinate system, where Eq. (1.6) has uniformly in $\varepsilon$ bounded coefficients, see Sect. 2.2. The effective equation has the form

$$
\dot{q}_{j}=\lambda \partial_{p_{j}} H^{\mathrm{res}}-\frac{q_{j}}{2}+\sqrt{\mathcal{T}_{j}} \dot{\beta}_{j}^{1}, \quad \dot{p}_{j}=-\lambda \partial_{q_{j}} H^{\mathrm{res}}-\frac{p_{j}}{2}+\sqrt{\mathcal{\mathcal { T }}_{j}} \dot{\beta}_{j}^{2}, \quad j \in \mathcal{C},
$$

where $\left(\beta_{j}^{k}\right)_{k=1,2, j \in \mathcal{C}}$ are standard independent Brownian motions, while $H^{\text {res }}(p, q)$ denotes the resonant averaging of the interaction potential $\frac{1}{2} \sum_{|k-j|=1} V\left(q_{j}, q_{k}\right)(\operatorname{see}(2.35)){ }^{4}$

Equation (1.10) has a unique solution, this solution is defined globally, and $(1.10)$ is mixing (see $[26,44,45]$ ). Let us fix $T \geq 0$ and some "not very bad" random initial conditions $\left(p_{0}, q_{0}\right) .{ }^{5}$ We prove

\footnotetext{
${ }^{4}$ Despite that the interaction potential does not depend on the moments $p_{k}$, its resonant averaging may depend on them.

${ }^{5}$ By "not very bad" we mean that the components $p_{0 j}^{2}, q_{0 j}^{2}$ have finite exponential moments which are bounded uniformly with respect to $j \in \mathcal{C}$ and with respect to the choice of the set $\mathcal{C} \subset \mathbb{Z}^{d}$. See ass. $H I$ in Sect. 1.4.
} 
Theorem 1.1. Let $\left(p^{\varepsilon}, q^{\varepsilon}\right)(\tau)$ and $(p, q)(\tau)$ be unique solutions of Eq. (1.6) and the effective equation (1.10), satisfying $\mathcal{D}\left(p^{\varepsilon}, q^{\varepsilon}\right)(0)=\mathcal{D}(p, q)(0)=\mathcal{D}\left(p_{0}, q_{0}\right)$. Then

$$
\mathcal{D}\left(I\left(p^{\varepsilon}, q^{\varepsilon}\right)(\cdot)\right) \rightarrow \mathcal{D}(I(p, q)(\cdot)) \quad \text { as } \quad \varepsilon \rightarrow 0
$$

on $C\left([0, T], \mathbb{R}^{|\mathcal{C}|}\right)$ uniformly in $\mathcal{C}$.

Theorem 1.1 is a corollary of Theorem 2.4. This is explained in Sect. 2.3. Here, $\mathcal{D}(\cdot)$ denotes the distribution of a random variable. By saying that a convergence is uniform in $\mathcal{C}$, we mean that it holds uniformly with respect to the choice of the finite set $\mathcal{C} \subset \mathbb{Z}^{d}$. In particular, with respect to the number of nodes $|\mathcal{C}|$. Uniformity in $\mathcal{C}$ of the weak convergences of measures through all the text is understood in the sense of finite-dimensional projections. It means that for any finite set of nodes $\Lambda \subset \mathbb{Z}^{d}$, projections of considered measures to $\Lambda$ converge with a rate, independent from the choice of the set $\mathcal{C} \supseteq \Lambda$. For instance, for convergence (1.11) this means

$$
\mathbf{E} f\left(I\left(p^{\varepsilon}, q^{\varepsilon}\right)(\cdot)\right) \rightarrow \mathbf{E} f(I(p, q)(\cdot)) \quad \text { as } \varepsilon \rightarrow 0 \quad \text { uniformly in } \mathcal{C},
$$

for any continuous bounded functional $f: C\left([0, T], \mathbb{R}^{|\mathcal{C}|}\right) \mapsto \mathbb{R}$, satisfying Supp $f \subseteq \Lambda$, where Supp $f$ is defined in Sect. 1.4, Agreements 6. Here and further on $\mathbf{E}$ denotes the expectation.

Remark 1.2. In a similar but technically more complicated way, it can be shown that the limiting (as $\varepsilon \rightarrow 0$ ) behaviour of joint actions $I$ and slow components on angles $\psi$ is also governed by the effective equation, see [29]. Since it is not needed for study of the limiting transport of energy, we skip it.

Let $\mu^{\varepsilon}$ be the unique stationary measure of Eq. (1.6). Each limiting point (as $\varepsilon \rightarrow 0$ ) of the family of measures $\left\{\mu^{\varepsilon}, 0<\varepsilon \leq 1\right\}$ is an invariant measure of Eqs. (1.3)-(1.5) with $\varepsilon=0$. This equation describes a system of uncoupled harmonic oscillators, so it has plenty of invariant measures. The next theorem ensures that, in fact, only one of them is the limiting point and distinguishes it.

Theorem 1.3. Let $\mu^{\varepsilon}$ and $\mu$ be the unique stationary measures of Eq. (1.6) and the effective equation (1.10) correspondingly. Then, $\mu^{\varepsilon} \rightarrow \mu$ as $\varepsilon \rightarrow 0$. Under some additional assumption, this convergence holds uniformly in $\mathcal{C}$.

See for details Theorem 2.8. Roughly speaking, the additional assumption above demands the effective equation for an infinite system of oscillators to have a unique stationary measure, in some class of measures. In particular, it holds if $\lambda$ is sufficiently small and second partial derivatives of the interaction potential $V$ are bounded.

To establish the uniformity in $\mathcal{C}$ of convergences from Theorems 1.1 and 1.3, we employ a method developed in [19]. Its main tool is Lemma 2.1, where using $l_{2}$-norms with exponential decaying weight, we obtain uniform in $\mathcal{C}, \varepsilon$ and $\tau$ estimates for solutions of Eq. (1.6).

In Appendix A, we prove that rate of mixing of Eq. (1.6) is independent from $\varepsilon$. Jointly with Theorems 1.1 and 1.3, this implies the following result. 
Theorem 1.4. (i) The convergence $\mathcal{D}\left(I\left(p^{\varepsilon}, q^{\varepsilon}\right)(\tau)\right) \rightarrow \mathcal{D}(I(p, q)(\tau))$ as $\varepsilon \rightarrow 0$ provided by Theorem 1.1 holds uniformly in $\tau \geq 0$.

(ii) We have

$$
\begin{aligned}
\lim _{\varepsilon \rightarrow 0} \lim _{\tau \rightarrow \infty} \mathcal{D}\left(I\left(p^{\varepsilon}, q^{\varepsilon}\right)(\tau)\right) & =\lim _{\tau \rightarrow \infty} \lim _{\varepsilon \rightarrow 0} \mathcal{D}\left(I\left(p^{\varepsilon}, q^{\varepsilon}\right)(\tau)\right) \\
& =\lim _{\substack{\varepsilon \rightarrow 0 \\
\tau \rightarrow \infty}} \mathcal{D}\left(I\left(p^{\varepsilon}, q^{\varepsilon}\right)(\tau)\right)=\Pi_{I *} \mu,
\end{aligned}
$$

where $\mu$ is the unique stationary measure of the effective equation (1.10) and $\Pi_{I}$ denotes the projection to the space of actions.

For details, see Theorem 2.9. Define the local energy of a $j$ th oscillator $\mathcal{E}_{j}^{\nu}(p, q):=\frac{p_{j}^{2}+q_{j}^{2}}{2}+\frac{\nu}{2} \sum_{k \in \mathcal{C}:|j-k|=1} V\left(q_{j}, q_{k}\right), \quad$ so that $H^{\nu}=\sum_{j \in \mathcal{C}} \mathcal{E}_{j}^{\nu}$.

Let $\left(p^{\varepsilon}, q^{\varepsilon}\right)(\tau)$ be a solution of Eq. (1.6). Since $\mathcal{E}_{j}^{\nu}=I_{j}+O(\nu)$, we obtain the following result whose full version is given in Proposition 2.10.

Proposition 1.5. The limiting (as $\varepsilon \rightarrow 0$ ) behaviour of the vector of local energy $\left(\mathcal{E}_{j}^{\nu}\left(p^{\varepsilon}, q^{\varepsilon}\right)\right)_{j \in \mathcal{C}}$ coincides with that of the vector of actions $I\left(p^{\varepsilon}, q^{\varepsilon}\right)$, while the latter is described by Theorems 1.1-1.4.

\subsection{Main Results: The Energy Transport}

Our next goal is to study the limiting (as $\varepsilon \rightarrow 0$ ) dynamics of a stationary Hamiltonian energy flow in system (1.6). We will assume that $\lambda$ is sufficiently small. ${ }^{6}$

The local energy $\mathcal{E}_{j}^{\nu}$ changes due to the Hamiltonian coupling of the $j$ th oscillator with neighbouring oscillators and with the $j$ th thermostat. Applying the Ito formula, we get

$\dot{\mathcal{E}}_{j}^{\nu}=\varepsilon^{-1}\left\{\mathcal{E}_{j}^{\nu}, H^{\nu}\right\}+(\text { thermost. term })_{j}=\frac{\lambda}{2} \sum_{k:|k-j|=1} \mathcal{J}_{k j}+$ (thermost. term $)_{j}$,

where $\{\cdot, \cdot\}$ denotes the Poisson bracket, (thermost. term) $)_{j}:=-p_{j}^{2}+\mathcal{T}_{j}+$ $\sqrt{2 \mathcal{T}_{j}} \dot{\beta}_{j}$, and

$\mathcal{J}_{k j}(p, q):=\left\{\frac{p_{j}^{2}+q_{j}^{2}}{2}-\frac{p_{k}^{2}+q_{k}^{2}}{2}, V\left(q_{j}, q_{k}\right)\right\}=p_{k} \partial_{q_{k}} V\left(q_{k}, q_{j}\right)-p_{j} \partial_{q_{j}} V\left(q_{k}, q_{j}\right)$

is the Hamiltonian energy flow from the $k$ th oscillator to the $j$ th one, normalized with respect to $\lambda$ (see [12]). Or, for short, the energy flow. We prove

Theorem 1.6. Let $\mu^{\varepsilon, \lambda}$ be the unique stationary measure of Eq. (1.6). Assume $\lambda$ to be sufficiently small. Then, under suitable assumptions for the interaction

\footnotetext{
${ }^{6}$ Here and further on the rate of smallness of $\lambda$ is independent neither from $\varepsilon$ nor from the choice of the set $\mathcal{C}$.
} 
potential $V$, there exists a $C^{1}$-smooth strictly positive function $\kappa: \mathbb{R}_{+}^{2} \mapsto \mathbb{R}_{+}$ satisfying $\kappa(x, y) \equiv \kappa(y, x)$, such that for every $j, k \in \mathcal{C},|j-k|=1$, we have

$$
\left\langle\mu^{\varepsilon, \lambda}, \mathcal{J}_{k j}\right\rangle \rightarrow \lambda \kappa\left(\mathcal{T}_{k}, \mathcal{T}_{j}\right)\left(\mathcal{T}_{k}-\mathcal{T}_{j}\right)+o(\lambda) \quad \text { as } \quad \varepsilon \rightarrow 0
$$

uniformly in $\mathcal{C}$, where $o(\lambda) / \lambda \rightarrow 0$ as $\lambda \rightarrow 0$ uniformly in $\mathcal{C}$.

For details, see Theorem 3.4. We emphasize that the function $\kappa\left(\mathcal{T}_{k}, \mathcal{T}_{j}\right)$, which we call the conductivity, depends only on the temperatures $\mathcal{T}_{k}, \mathcal{T}_{j}$, and not on the choice of the set $\mathcal{C}$. Convergence (1.15) shows that in the stationary regime the averaged flow of energy is approximately proportional (uniformly in $\mathcal{C})$ to the local temperature gradient, if the noise and the interaction between oscillators are sufficiently weak. The coefficient of proportionality $\kappa$ is strictly positive and depends on the temperatures in a sufficiently smooth way. To prove Theorem 1.6, we note that Theorem 1.3 implies $\left\langle\mu^{\varepsilon, \lambda}, \mathcal{J}_{k j}\right\rangle \rightarrow\left\langle\mu^{\lambda}, \mathcal{J}_{k j}\right\rangle$ as $\varepsilon \rightarrow 0$ uniformly in $\mathcal{C}$, where $\mu^{\lambda}$ is the unique stationary measure of the effective equation (1.10). Then, we analyse the development of the measure $\mu^{\lambda}$ in $\lambda$.

Remark 1.7. For the reader interested in the limiting (as $\varepsilon \rightarrow 0$ ) behaviour of the stationary thermostatic energy flow, we note that it can be easily obtained from Eq. (1.13). Indeed, let us average the both sides of (1.13) with respect to the stationary measure $\mu^{\varepsilon, \lambda}$. The left-hand side vanishes, while the limiting behaviour of the first term in the right-hand side is given in Theorem 1.6.

Now for simplicity, we fix the dimension of the lattice $d=1$ and put $\mathcal{C}=\{0,1, \ldots, N\}$. Let us study the limit $N \rightarrow \infty$. For $x \in[0,1]$ denote $j_{x}:=[x N]$ and $\mathcal{J}(x):=\mathcal{J}_{j_{x}+1 j_{x}}$. If we put the oscillators to the interval $[0,1]$, a $j$ th oscillator to the point $j / N$, then the function $\mathcal{J}(x)$ will describe the energy flow between the nearest to the point $x$ oscillators, so it can be seen as the energy flow through the point $x$. Choose a temperature profile $\mathcal{T}_{j}:=\mathcal{T}(j / N)$, where $\mathcal{T}(x)$ is a $C^{1}$-smooth positive function, defined for $x \in[0,1]$. Then, Theorem 1.6 immediately implies

$$
\lim _{N \rightarrow \infty} \lim _{\lambda \rightarrow 0} \lim _{\varepsilon \rightarrow 0} \frac{N}{\lambda}\left\langle\mu^{\varepsilon, \lambda}, \mathcal{J}(x)\right\rangle=\hat{\kappa}(\mathcal{T}(x)) \frac{\mathrm{d}}{\mathrm{d} x} \mathcal{T}(x), \quad \text { where } \hat{\kappa}(y):=\kappa(y, y) .
$$

If the temperature profile is linear, i.e. $\mathcal{T}(x)=\mathcal{T}_{1} x+\mathcal{T}_{0}(1-x)$, where $\mathcal{T}_{0}, \mathcal{T}_{1}>0$, then (1.16) implies that

$$
\lim _{N \rightarrow \infty} \lim _{\lambda \rightarrow 0} \lim _{\varepsilon \rightarrow 0} \frac{N}{\lambda \delta \mathcal{T}}\left\langle\mu^{\varepsilon, \lambda}, \mathcal{J}(x)\right\rangle=\hat{\kappa}(\mathcal{T}(x)),
$$

where $\delta \mathcal{T}:=\mathcal{T}_{1}-\mathcal{T}_{0}$. This resembles the Fourier law, see [12].

Next, we study stationary space-time correlations of the energy flow. In particular, we get the following result.

Theorem 1.8. For any $\hat{\mathcal{T}}>0$ and $N \in \mathbb{N}$, we have

$$
\hat{\kappa}(\hat{\mathcal{T}})=\lim _{\lambda \rightarrow 0} \lim _{\varepsilon \rightarrow 0} \frac{1}{\hat{\mathcal{T}}^{2} N} \int_{0}^{\infty}\left\langle\mu^{\varepsilon, \lambda}, \sum_{j=0}^{N-1} \mathcal{J}_{j j+1} \mathfrak{P}_{\tau}^{\varepsilon, \lambda}\left(\sum_{j=0}^{N-1} \mathcal{J}_{j j+1}\right)\right\rangle d \tau
$$


where $\mathfrak{P}_{\tau}^{\varepsilon, \lambda}$ denotes the Markov semigroup associated with Eq. (1.6) with the temperatures satisfying $\mathcal{T}_{j}=\hat{\mathcal{T}}$ for every $j \in \mathcal{C}$, while $\mu^{\varepsilon, \lambda}$ denotes its unique stationary measure (which is the Gibbs measure at the temperature $\hat{\mathcal{T}}$ ).

See for details Sect. 3.3. Relation (1.18) resembles the Green-Kubo formula, see $[12,32,41] .^{7}$ To prove Theorem 1.8 , we employ the averaging technique developed above, an explicit formula for the conductivity $\kappa$, given in Theorem 3.4 (which is a full version of Theorem 1.6) and the uniformity in $\varepsilon$ of the rate of mixing for Eq. (1.6).

Despite that (1.17) and (1.18) resemble the Fourier law and the GreenKubo formula, of course they are not.

\subsection{Assumptions and Agreements}

Assumptions. Throughout the text, we assume that the following hypotheses hold.

HV. The function $V: \mathbb{R}^{2} \rightarrow \mathbb{R}$ is $C^{r}$-smooth, $r \geq 2$, and $V(x, y)=V(y, x)$ for any $x, y \in \mathbb{R}$. There exists a constant $C>0$ such that

$$
\begin{gathered}
|V(x, y)| \leq C\left(1+x^{2}+y^{2}\right) \\
\left|\partial_{z_{1}} V(x, y)\right| \leq C(1+|x|+|y|), \quad\left|\partial_{z_{1} z_{2}}^{2} V(x, y)\right| \leq C\left(1+x^{2}+y^{2}\right),
\end{gathered}
$$

where $z_{1}, z_{2} \in\{x, y\}$. Moreover, higher orders partial derivatives of $V$ have at most a polynomial growth at infinity.

HI. There exist constants $\alpha_{0}>0$ and $C>0$, independent from the choice of the set $\mathcal{C}$, such that the initial conditions $\left(p_{0}, q_{0}\right)$ satisfy

$$
\mathbf{E} \mathrm{e}^{\alpha_{0}\left(p_{0 j}^{2}+q_{0 j}^{2}\right)}<C \text { for every } j \in \mathcal{C} .
$$

Remark 1.9. We use relations (1.19), (1.20) only to obtain uniform in $\mathcal{C}, \varepsilon$ and $\tau$ a priori estimates (2.1) for solutions and stationary measures of Eq. (1.6), and to prove that Eq. (1.6) is mixing with independent from $\varepsilon$ rate. Once these properties are given, we do not need more (1.19), (1.20).

More specifically, for Theorem 1.1, we need the first estimate from (2.1) to be held. For Theorems 1.3 and 1.6, we need (2.1) and uniqueness of the stationary measure for Eq. (1.6). For Theorems 1.4 and 1.8, we need the same and, additionally, we need Eq. (1.6) to be mixing with independent from $\varepsilon$ rate.

Note that the exponential estimates (2.1) are superfluous. It suffices to have polynomial estimates of sufficiently large degree. Moreover, if we have only estimates which are not uniform in $\mathcal{C}$, then all the results remain true except the uniformity of convergences in $\mathcal{C}$.

Agreements

1. We refer to item (i) of Theorem 1.4 as Theorem 1.4.i, etc.

\footnotetext{
${ }^{7}$ In formula (1.18), we could also send $N$ to infinity; however, it does not make sense: limit (1.18) is the same for all $N$.
} 
2. By $C, C_{1}, C_{2}, \ldots$, we denote various positive constants and by $C(a), C_{1}(a), \ldots$-positive constants which depend on a parameter $a$. Sometimes, we skip the dependence of the constants on parameters. However, unless otherwise stated, we always indicate if they depend on the choice of the set $\mathcal{C}$, times $t, s, \tau, \ldots$, positions $j, k, l, m, \ldots \in \mathcal{C}$ and small parameters $\varepsilon, \lambda$. Constants $C, C(a), \ldots$ can change from formula to formula.

3. We use notations $a \wedge b:=\min (a, b), a \vee b=\max (a, b)$.

4. For $M \in \mathbb{N}$ and vectors $a=\left(a_{k}\right), b=\left(b_{k}\right) \in \mathbb{C}^{M}$, by $a \cdot b$ we denote the Euclidean scalar product in $\mathbb{C}^{M} \simeq \mathbb{R}^{2 M}$, and by $|a|$ the corresponding norm, ${ }^{8}$

$$
\begin{aligned}
& a \cdot b:=\sum_{k}\left(\operatorname{Re} a_{k} \operatorname{Re} b_{k}+\operatorname{Im} a_{k} \operatorname{Im} b_{k}\right)=\sum_{k} \operatorname{Re} a_{k} \bar{b}_{k} \\
& \text { and }|a|^{2}:=a \cdot a=\sum_{k}\left|a_{k}\right|^{2} \text {. }
\end{aligned}
$$

5. By saying that a function $f: \mathbb{C}^{M} \mapsto \mathbb{R}$ is $C^{n}$-smooth, we mean that it is $C^{n}$-smooth as a function $f: \mathbb{R}^{2 M} \mapsto \mathbb{R}$.

6. Let $X$ be some vector space and $f:\left(x_{j}\right)_{j \in \mathcal{C}} \in X^{|\mathcal{C}|} \mapsto \mathbb{R}$. By $\operatorname{Supp} f$, we denote a minimal set $S \subseteq \mathcal{C}$ such that the function $f$ is independent from $\left(x_{j}\right)_{j \notin S}$.

7. For a metric space $X$ by $\mathcal{L}_{b}(X)\left(\mathcal{L}_{\text {loc }}(X)\right)$, we denote the space of bounded Lipschitz continuous (locally Lipschitz continuous) functions from $X$ to $\mathbb{R}$.

8. Assertions of the type "something is sufficiently small/sufficiently close to one/..." always assume an estimate independent from the choice of the set $\mathcal{C}$.

9. We assume $0<\varepsilon \leq 1$ to be sufficiently small where it is needed.

\section{The Limiting Dynamics}

In this section, we study the limiting as $\varepsilon \rightarrow 0$ behaviour of solutions of Eq. (1.6). Most of results of this section are independent from the size of $\lambda$, where $0<\lambda \leq 1$.

\subsection{Estimates on Solutions}

It is well known that Eq. (1.6) has a unique solution and this solution is defined for all $\tau \geq 0$ (see [26]). In Appendix A, we prove that Eq. (1.6) is mixing. First, we need to obtain estimates for its solution and stationary measure, which are uniform in $\varepsilon, \tau$ and $\mathcal{C}$.

\footnotetext{
${ }^{8}$ We denote the $l_{2}$-norm in the space of complex vectors $\mathbb{C}^{M}$ and the $l_{1}$-norm in the space of indices $\mathbb{Z}^{d}$ by the same sign $|\cdot|$. This will not cause misunderstanding.
} 
Lemma 2.1. There exist constants $\alpha>0, \varepsilon_{0}>0$ and $C>0$, independent from the choice of the set $\mathcal{C}$, such that for all $0<\varepsilon<\varepsilon_{0}, 0<\lambda \leq 1, j \in \mathcal{C}$ and $\tau \geq 0$ we have
(i) $\mathbf{E} \max _{s \in[\tau, \tau+1]} \mathrm{e}^{\alpha\left(p_{j}^{2}(s)+q_{j}^{2}(s)\right)}<C$,
(ii) $\left\langle\mu, \mathrm{e}^{\alpha\left(p_{j}^{2}+q_{j}^{2}\right)}\right\rangle<C$,

where $(p, q)(\tau)$ is a solution of $E q .(1.6)$, satisfying $\mathcal{D}(p, q)(0)=\mathcal{D}\left(p_{0}, q_{0}\right)$, and $\mu$ is its unique stationary measure.

Proof. Item (ii) follows from item (i), the mixing property of Eq. (1.6) and the Fatou lemma. ${ }^{9}$ Let us now explain the idea of the proof of item (i). Fix some $1 / 2<\gamma<1$, and denote

$$
U^{j}(p, q):=\sum_{k \in \mathcal{C}} \gamma^{|k-j|}\left(\mathcal{E}_{k}(p, q)+\frac{\varepsilon}{2} p_{k} q_{k}\right), \quad j \in \mathbb{Z}^{d},
$$

where we recall that the local energy $\mathcal{E}_{k}$ is defined in (1.12). ${ }^{10}$ Estimate (2.1).i with the constant $C$ depending on the number of nodes $|\mathcal{C}|$ can be obtained in a standard way, by applying the Ito formula to the function $\left.\mathrm{e}^{\alpha U^{j}}\right|_{\gamma=1}$, see (2.9). To derive it, one should crucially use that for $\gamma=1$ the function $\mathcal{E}^{j}:=\sum_{k \in \mathcal{C}} \gamma^{|k-j|} \mathcal{E}_{k}(p, q)$ coincides with the Hamiltonain $H$, so that $\left.\mathcal{E}^{j}\right|_{\gamma=1}$ is conserved by the Hamiltonian flow. The obtained in such a way estimate is not uniform in $\mathcal{C}$ because the r.h.s. of (2.9) contains terms proportional to the sum $\sum_{k \in \mathcal{C}} \gamma^{|j-k|}$, which equals to $|\mathcal{C}|$ if $\gamma=1$. To overcome this difficulty, we put $\gamma<1$, so that the sum above is bounded uniformly in $\mathcal{C}$. Despite that in this case the function $\mathcal{E}^{j}$ is not a first integral of the Hamiltonain flow, the nearest-neighbour character of interaction between oscillators provides that if $\gamma \sim 1, \mathcal{E}^{j}$ is its "approximate integral", in the sense that $\varepsilon^{-1}\left\{\mathcal{E}^{j}, H\right\} \sim(1-\gamma)$, see (2.13). This suffices to establish the desired estimate.

Now we start the proof. First, we will establish the following auxiliary proposition. Introduce the family of norms

$$
\|x\|_{j}^{2}:=\sum_{k \in \mathcal{C}} \gamma^{|j-k|} x_{k}^{2}, \quad \text { where } j \in \mathbb{Z}^{d} \text { and } \quad x=\left(x_{k}\right)_{k \in \mathcal{C}} \in \mathbb{R}^{|\mathcal{C}|} .
$$

Proposition 2.2. There exists $\varepsilon_{1}>0$ such that for any $1 / 2<\gamma<1, j \in \mathbb{Z}^{d}$ and $0<\varepsilon<\varepsilon_{1}$, we have

$$
\left(\|p\|_{j}^{2}+\|q\|_{j}^{2}\right) / 4 \leq U^{j}(p, q) \leq C\left(\|p\|_{j}^{2}+\|q\|_{j}^{2}\right)+C_{1}(\gamma), \quad(p, q) \in \mathbb{R}^{2|\mathcal{C}|} .
$$

Proof. Let us prove the upper bound. Since (1.19) implies $\mathcal{E}_{k} \leq \frac{p_{k}^{2}}{2}$ $+C \sum_{l:|l-k| \leq 1} q_{l}^{2}+C$, we have

\footnotetext{
${ }^{9}$ In fact, it can be obtained even without employing of the mixing property, in a slightly more complicated way (see Section 2.5.2 from [31]). One should use that dynamics "forgets" initial conditions (see (2.22)), and the Fatou lemma.

${ }^{10}$ For the brevity of notations here and further on in this proof, we skip the upper index $\nu$.
} 


$$
\begin{aligned}
U^{j} & \leq \sum_{k \in \mathcal{C}} \gamma^{|j-k|}\left(\frac{p_{k}^{2}}{2}+C \sum_{l:|l-k| \leq 1} q_{l}^{2}+C+\frac{p_{k}^{2}+q_{k}^{2}}{2}\right) \\
& \leq \sum_{k \in \mathcal{C}} \gamma^{|j-k|}\left(p_{k}^{2}+C_{1} \sum_{l:|l-k| \leq 1} q_{l}^{2}+C\right) .
\end{aligned}
$$

Since $\gamma>1 / 2$, we have

$$
\sum_{k \in \mathcal{C}} \gamma^{|j-k|} \sum_{l:|l-k| \leq 1} q_{l}^{2} \leq 2 \sum_{k \in \mathcal{C}} \sum_{l:|l-k| \leq 1} \gamma^{|j-l|} q_{l}^{2} \leq C\|q\|_{j}^{2}
$$

Moreover, $\sum_{k \in \mathcal{C}} \gamma^{|j-k|} \leq \sum_{m \in \mathbb{Z}^{d}} \gamma^{|m|} \leq C(\gamma)$. Then, we obtain the desired estimate. The lower bound follows from (1.19) and smallness of $\varepsilon$ in a similar way.

Now let us formulate the following result, which we will establish after the end of the proof of the lemma.

Lemma 2.3. Let $1 / 2<\gamma<1$ be sufficiently close to one and $0<\varepsilon_{0}<\varepsilon_{1}$ be sufficiently small, where $\varepsilon_{1}$ is defined in Proposition 2.2. Fix any $\delta_{0}>0$. Then, for $0<\delta<\delta_{0}$ sufficiently small and any solution $(p, q)(\tau)$ of Eq. (1.6), satisfying for some $j \in \mathbb{Z}^{d}$ the estimate

$$
\mathbf{E} \mathrm{e}^{\delta_{0} U^{j}(p, q)(0)}<C,
$$

we have

$$
\mathbf{E} \max _{s \in[\tau, \tau+1]} \mathrm{e}^{\delta U^{j}(p, q)(s)}<C_{1}
$$

for any $0<\varepsilon<\varepsilon_{0}, 0<\lambda \leq 1$ and $\tau \geq 0$, where $C_{1}=C_{1}\left(\gamma, \varepsilon_{0}, \delta, C\right)$.

We fix the parameters $\gamma$ and $\varepsilon_{0}$ as in Lemma 2.3 and do not indicate dependence of constants from them. Using first the upper bound from Proposition 2.2 and then the Jensen inequality joined with ass. $H I$, we see that

$$
\mathbf{E} \mathrm{e}^{\delta_{0} U^{j}\left(p_{0}, q_{0}\right)} \leq C_{1} \mathbf{E} \mathrm{e}^{\delta_{0} C\left(\left\|p_{0}\right\|_{j}^{2}+\left\|q_{0}\right\|_{j}^{2}\right)}<C_{2},
$$

for $\delta_{0}$ sufficiently small and any $j \in \mathbb{Z}^{d}$. Since $p_{j}^{2}+q_{j}^{2} \leq\|p\|_{j}^{2}+\|q\|_{j}^{2}$, the lower bound from Proposition 2.2 joined with Lemma 2.3 implies that

$$
\mathbf{E} \max _{s \in[\tau, \tau+1]} \mathrm{e}^{\delta\left(p_{j}^{2}(s)+q_{j}^{2}(s)\right) / 4} \leq \mathbf{E} \max _{s \in[\tau, \tau+1]} \mathrm{e}^{\delta U^{j}(p, q)(s)}<C,
$$

for $\delta>0$ sufficiently small. Since the constant $C_{2}$ from (2.6) is independent from $j$, the constant $C$ from (2.7) also is, so that we get the desired estimate with $\alpha:=\delta / 4$.

Proof of Lemma 2.3. Without loss of generality, we assume that $j=0$ and skip the index $j$ in the notations for $U^{j}$ and $\|\cdot\|_{j}$. Moreover, for simplicity we put $\lambda=1$ : analysing the proof below, it is easy to see that all estimates we obtain are uniform in $0<\lambda \leq 1$.

Step 1. First, we will find $\gamma, \varepsilon_{0}$ and $\delta_{1}>0$ such that for any $0<\varepsilon<\varepsilon_{0}$ and $s \geq 0$ we have

$$
\mathbf{E} \mathrm{e}^{\delta_{1} U(s)} \leq C,
$$


where we have denoted $U(s):=U(p, q)(s)$. Further on, we present only formal computation which could be justified by standard stopping-time arguments (see e.g. [25]). Applying the Ito formula to $\mathrm{e}^{\delta_{1} U(s)}$, we obtain

$$
\begin{aligned}
\frac{\mathrm{d}}{\mathrm{d} s} \mathrm{e}^{\delta_{1} U(s)} & =\varepsilon^{-1}\left\{\mathrm{e}^{\delta_{1} U(s)}, H\right\}-\sum_{l \in \mathcal{C}} p_{l} \partial_{p_{l}} \mathrm{e}^{\delta_{1} U(s)}+\sum_{l \in \mathcal{C}} \mathcal{T}_{l} \partial_{p_{l}^{2}}^{2} \mathrm{e}^{\delta_{1} U(s)}+\frac{\mathrm{d}}{\mathrm{d} s} M_{s_{0}, s} \\
& =\delta_{1} \mathrm{e}^{\delta_{1} U(s)}\left(\mathcal{Y}_{1}+\mathcal{Y}_{2}+\mathcal{Y}_{3}\right)+\frac{\mathrm{d}}{\mathrm{d} s} M_{s_{0}, s}
\end{aligned}
$$

where

$$
\mathcal{Y}_{1}=\varepsilon^{-1}\{U, H\}, \quad \mathcal{Y}_{2}=-\sum_{l \in \mathcal{C}} p_{l} \partial_{p_{l}} U, \quad \mathcal{Y}_{3}=\sum_{l \in \mathcal{C}} \mathcal{T}_{l}\left(\partial_{p_{l}^{2}}^{2} U+\delta_{1}\left(\partial_{p_{l}} U\right)^{2}\right)
$$

and the martingal $M_{s_{0}, s}:=\delta_{1} \int_{s_{0}}^{s} \mathrm{e}^{\delta_{1} U(s)} \sum_{l \in \mathcal{C}} \sqrt{2 \mathcal{T}_{l}} \partial_{p_{l}} U \mathrm{~d} \beta_{l}$, where $s_{0} \leq s$. Let us estimate the terms $\mathcal{Y}_{1}, \mathcal{Y}_{2}, \mathcal{Y}_{3}$ separately.

Term $\mathcal{Y}_{1}$. Writing $\mathcal{Y}_{1}$ in more details, we obtain

$$
\mathcal{Y}_{1}=\varepsilon^{-1} \sum_{k \in \mathcal{C}} \gamma^{|k|}\left\{\mathcal{E}_{k}, H\right\}+\sum_{k \in \mathcal{C}} \frac{\gamma^{|k|}}{2}\left\{p_{k} q_{k}, H\right\}
$$

We have

$$
\begin{aligned}
\varepsilon^{-1}\left\{\mathcal{E}_{k}, H\right\} & =\left\{\frac{p_{k}^{2}}{2}, \frac{1}{2} \sum_{|l-m|=1} V\left(q_{m}, q_{l}\right)\right\}+\left\{\frac{1}{2} \sum_{l:|l-k|=1} V\left(q_{k}, q_{l}\right), \sum_{m \in \mathcal{C}} \frac{p_{m}^{2}}{2}\right\} \\
& =\left\{\frac{p_{k}^{2}}{2}, \sum_{l:|l-k|=1} V\left(q_{k}, q_{l}\right)\right\}+\frac{1}{2} \sum_{l:|l-k|=1}\left\{V\left(q_{k}, q_{l}\right), \frac{p_{k}^{2}+p_{l}^{2}}{2}\right\} \\
& =\sum_{l:|l-k|=1}\left\{\frac{p_{k}^{2}-p_{l}^{2}}{4}, V\left(q_{k}, q_{l}\right)\right\} .
\end{aligned}
$$

Note that for any $\left(x_{k l}\right)_{k, l \in \mathcal{C}} \in \mathbb{R}^{|\mathcal{C} \times \mathcal{C}|}$, we have $\sum_{k \in \mathcal{C}} \sum_{l:|l-k|=1} x_{k l}=$ $\frac{1}{2} \sum_{|l-k|=1}\left(x_{k l}+x_{l k}\right)$. Using $(2.11)$ and applying the latter identity with $x_{k l}=$ $\gamma^{|k|}\left\{\frac{p_{k}^{2}-p_{l}^{2}}{4}, V\left(q_{k}, q_{l}\right)\right\}$, we find

$$
\begin{aligned}
\varepsilon^{-1} \sum_{k \in \mathcal{C}} \gamma^{|k|}\left\{\mathcal{E}_{k}, H\right\} & =\sum_{k \in \mathcal{C}} \sum_{l:|l-k|=1} \gamma^{|k|}\left\{\frac{p_{k}^{2}-p_{l}^{2}}{4}, V\left(q_{k}, q_{l}\right)\right\} \\
& =\frac{1}{2} \sum_{|l-k|=1}\left(\gamma^{|k|}-\gamma^{|l|}\right)\left\{\frac{p_{k}^{2}-p_{l}^{2}}{4}, V\left(q_{k}, q_{l}\right)\right\}
\end{aligned}
$$


Since for $k, l \in \mathcal{C}$ satisfying $|l-k|=1$ we have $|l|-|k|= \pm 1$, we obtain

$$
\begin{aligned}
\left|\gamma^{|k|}-\gamma^{|l|}\right| & =\gamma^{|k|}\left|1-\gamma^{|l|-|k|}\right| \\
& =\left\{\begin{array}{cl}
\gamma^{|k|}(1-\gamma), & \text { if }|l|-|k|=1 \\
\gamma^{|k|} \gamma^{-1}(1-\gamma), & \text { if }|l|-|k|=-1
\end{array} \leq 2 \gamma^{|k|}(1-\gamma),\right.
\end{aligned}
$$

where we have used that $1 / 2<\gamma<1$. In view of the estimate (1.20), we find $\left|\left\{\frac{p_{k}^{2}-p_{l}^{2}}{4}, V\left(q_{k}, q_{l}\right)\right\}\right| \leq C\left(p_{k}^{2}+p_{l}^{2}+q_{k}^{2}+q_{l}^{2}+1\right)$. Then, from (2.12), we get

$$
\begin{aligned}
\varepsilon^{-1} \sum_{k \in \mathcal{C}} \gamma^{|k|}\left\{\mathcal{E}_{k}, H\right\} & \leq(1-\gamma) C \sum_{|l-k|=1} \gamma^{|k|}\left(p_{k}^{2}+p_{l}^{2}+q_{k}^{2}+q_{l}^{2}+1\right) \\
& \leq(1-\gamma) C_{1}\left(\|p\|^{2}+\|q\|^{2}\right)+C_{2}(\gamma) .
\end{aligned}
$$

Let us estimate the term $\sum_{k \in \mathcal{C}} \frac{\gamma^{|k|}}{2}\left\{p_{k} q_{k}, H\right\}$ from (2.10). We have

$$
\left\{p_{k} q_{k}, H\right\}=p_{k} \partial_{p_{k}} H-q_{k} \partial_{q_{k}} H=p_{k}^{2}-q_{k}^{2}-\varepsilon \sum_{l:|l-k|=1} q_{k} \partial_{q_{k}} V\left(q_{k}, q_{l}\right) .
$$

Then, using that $\left|q_{k} \partial_{q_{k}} V\left(q_{k}, q_{l}\right)\right| \leq C\left(q_{k}^{2}+q_{l}^{2}+1\right)$, we find

$$
\begin{aligned}
\sum_{k \in \mathcal{C}} \frac{\gamma^{|k|}}{2}\left\{p_{k} q_{k}, H\right\} & \leq \frac{1}{2}\left(\|p\|^{2}-\|q\|^{2}\right)+C \varepsilon \sum_{k \in \mathcal{C}} \sum_{l:|l-k|=1} \gamma^{|k|}\left(q_{k}^{2}+q_{l}^{2}+1\right) \\
& \leq\|p\|^{2} / 2+\left(-1 / 2+C_{1} \varepsilon\right)\|q\|^{2}+C_{2}(\gamma)
\end{aligned}
$$

Combining (2.13) and (2.14), we obtain

$$
\mathcal{Y}^{1} \leq((1-\gamma) C+1 / 2)\|p\|^{2}+((1-\gamma+\varepsilon) C-1 / 2)\|q\|^{2}+C_{1}(\gamma) .
$$

Term $\mathcal{Y}_{2}$. Since

we find

$$
\partial_{p_{l}} U=\gamma^{|l|}\left(p_{l}+\frac{\varepsilon}{2} q_{l}\right)
$$

$$
\mathcal{Y}_{2}=-\|p\|^{2}-\frac{\varepsilon}{2} \sum_{l \in \mathcal{C}} \gamma^{|l|} p_{l} q_{l} \leq(-1+\varepsilon)\|p\|^{2}+\varepsilon\|q\|^{2} .
$$

Term $\mathcal{Y}_{3}$. In view of (2.16), we have $\partial_{p_{l}^{2}}^{2} U=\gamma^{|l|}$. Then, using that the temperatures $\mathcal{T}_{l}$ are bounded uniformly in $l \in \mathcal{C}$ (see (1.2)), we find

$$
\mathcal{Y}_{3}=\sum_{l \in \mathcal{C}} \mathcal{T}_{l}\left(\gamma^{|l|}+\delta_{1} \gamma^{2|l|}\left(p_{l}+\frac{\varepsilon}{2} q_{l}\right)^{2}\right) \leq C(\gamma)+2 \delta_{1}\left(\|p\|^{2}+\|q\|^{2}\right) .
$$

Substituting (2.15), (2.17) and (2.18) to (2.9), we get

$$
\frac{\mathrm{d}}{\mathrm{d} s} \mathrm{e}^{\delta_{1} U} \leq \delta_{1} \mathrm{e}^{\delta_{1} U}\left(\Delta\left(\|p\|^{2}+\|q\|^{2}\right)+C(\gamma)\right)+\frac{\mathrm{d}}{\mathrm{d} s} M_{s_{0}, s}
$$

where

$\Delta\left(\gamma, \varepsilon, \delta_{1}\right):=\left((1-\gamma) C+1 / 2-1+\varepsilon+2 \delta_{1}\right) \vee\left((1-\gamma+\varepsilon) C-1 / 2+\varepsilon+2 \delta_{1}\right)$. 
Fix $1 / 2<\gamma<1$ sufficiently close to one, $\varepsilon_{0}>0$ and $0<\delta_{1}<\delta_{0}$ sufficiently small, in such a way that $\Delta\left(\gamma, \varepsilon, \delta_{1}\right)<0$ for $\varepsilon \leq \varepsilon_{0}$. Then, using Proposition 2.2 , for $\varepsilon \leq \varepsilon_{0}$ we obtain

$$
\frac{\mathrm{d}}{\mathrm{d} s} \mathrm{e}^{\delta_{1} U} \leq \delta_{1} \mathrm{e}^{\delta_{1} U}\left(\Delta_{0} U+C\right)+\frac{\mathrm{d}}{\mathrm{d} s} M_{s_{0}, s},
$$

for some $\Delta_{0}<0$, where we do not more indicate dependence of constants on the parameters $\gamma, \varepsilon_{0}$ and $\delta_{1}$. Then, for an appropriate constant $C$, we get

$$
\frac{\mathrm{d}}{\mathrm{d} s} \mathrm{e}^{\delta_{1} U} \leq-\mathrm{e}^{\delta_{1} U}+C+\frac{\mathrm{d}}{\mathrm{d} s} M_{s_{0}, s} .
$$

Fixing $s_{0}=0$, taking the expectation and applying the Gronwall inequality to (2.21), we obtain

$$
\mathbf{E} \mathrm{e}^{\delta_{1} U(s)} \leq \mathbf{E} \mathrm{e}^{\delta_{1} U(0)} \mathrm{e}^{-s}+C
$$

so that we have (2.8).

Step 2. Now, we will prove (2.5) for $0<\delta<\delta_{1} / 2$. Integrating inequality (2.21) with $\delta_{1}$ replaced by $\delta$ over the interval $[\tau, s]$, where $\tau \leq s \leq \tau+1$, putting $s_{0}=\tau$ and taking $\mathbf{E} \max _{s \in[\tau, \tau+1]}$, we get

$$
\mathbf{E} \max _{s \in[\tau, \tau+1]} \mathrm{e}^{\delta U(s)} \leq \mathbf{E} \mathrm{e}^{\delta U(\tau)}+C+\mathbf{E} \max _{s \in[\tau, \tau+1]} M_{\tau, s} \leq C_{1}+\mathbf{E} \max _{s \in[\tau, \tau+1]} M_{\tau, s},
$$

where we have used (2.8). The Doob-Kolmogorov inequality ensures that

$$
\mathbf{E} \max _{s \in[\tau, \tau+1]} M_{\tau, s} \leq C \mathbf{E} \sqrt{[M]_{\tau, \tau+1}} \leq C \sqrt{\mathbf{E}[M]_{\tau, \tau+1}},
$$

where $[M]_{\tau, s}$ denotes the quadratic variation of the martingale $M_{\tau, s}$. Due to $(2.16)$,

$$
\begin{aligned}
{[M]_{\tau, \tau+1} } & =\delta^{2} \int_{\tau}^{\tau+1} \mathrm{e}^{2 \delta U} \sum_{l \in \mathcal{C}} 2 \mathcal{T}_{l} \gamma^{2|l|}\left(p_{l}+\frac{\varepsilon}{2} q_{l}\right)^{2} \mathrm{~d} s \\
& \leq C \int_{\tau}^{\tau+1} \mathrm{e}^{2 \delta U} \sum_{l \in \mathcal{C}} \gamma^{2|l|}\left(p_{l}^{2}+q_{l}^{2}\right) \mathrm{d} s \\
& \leq 4 C \int_{\tau}^{\tau+1} \mathrm{e}^{2 \delta U} U \mathrm{~d} s,
\end{aligned}
$$

in view of the lower bound from Proposition 2.2 and the estimate $\|p\|^{2}+\|q\|^{2} \geq$ $\sum_{l \in \mathcal{C}} \gamma^{2|l|}\left(p_{l}^{2}+q_{l}^{2}\right)$. Since $2 \delta<\delta_{1}$, estimate (2.8) provides $\mathbf{E}[M]_{\tau, \tau+1} \leq C$, so that (2.23) joined with (2.24) implies the desired relation (2.5).

\section{2. $a$-Variables and the Effective Equation}

Instead of studying Eq. (1.6) in the real $(p, q)$-coordinates, it is convenient to pass to the complex variables

$$
u=\left(u_{j}\right)_{j \in \mathcal{C}} \in \mathbb{C}^{|\mathcal{C}|}, \quad u_{j}(p, q)=p_{j}+i q_{j}, \quad j \in \mathcal{C} .
$$


Then, the actions and angles take the form

$$
I=\left(I_{j}\right)_{j \in \mathcal{C}}, I_{j}(u)=\frac{\left|u_{j}\right|^{2}}{2} \text { and } \varphi=\left(\varphi_{j}\right)_{j \in \mathcal{C}}, \varphi_{j}(u)=\arg u_{j} .
$$

Denote

$$
V_{j k}(u):=V\left(q_{j}(u), q_{k}(u)\right)=V\left(\operatorname{Im} u_{j}, \operatorname{Im} u_{k}\right),
$$

where $j, k \in \mathcal{C}$ satisfy $|k-j|=1$. Then, in the $u$-variables, Eq. (1.6) takes the form

where $^{11}$

$$
\dot{u}_{j}=\varepsilon^{-1} i u_{j}+P_{j}(u)+\sqrt{2 \mathcal{T}_{j}} \dot{\beta}_{j}
$$

$$
P=\left(P_{j}\right)_{j \in \mathcal{C}}, \quad P_{j}(u):=\lambda \sum_{k \in \mathcal{C}:|j-k|=1} i \nabla_{j} V_{j k}(u)-\operatorname{Re} u_{j},
$$

and $\nabla_{j}:=2 \partial_{\bar{u}_{j}}$ is the gradient with respect to the Euclidean scalar product in $\mathbb{C} \simeq \mathbb{R}^{2}$. The derivative $\partial_{\bar{u}_{j}}$ is understood formally, $\partial_{\bar{u}_{j}}:=\left(\partial_{p_{j}}+i \partial_{q_{j}}\right) / 2$.

Let $u^{\varepsilon}(\tau)$ be a unique solution of Eq. (2.25) satisfying $\mathcal{D}\left(u^{\varepsilon}(0)\right)=\mathcal{D}\left(u_{0}\right)$, where $u_{0}:=p_{0}+i q_{0}$. Our first aim is to study its limiting behaviour as $\varepsilon \rightarrow 0$. In the main order with respect to $\varepsilon$, Eq. (2.25) describes a system of fast synchronously rotating uncoupled oscillators [see also $\varphi$-equation from (1.8)]. Let us pass to a rotating coordinate system where the oscillators are immovable. Put

$$
a^{\varepsilon}=\left(a_{j}^{\varepsilon}\right)_{j \in \mathcal{C}}, \quad a_{j}^{\varepsilon}(\tau):=\mathrm{e}^{-i \varepsilon^{-1} \tau} u_{j}^{\varepsilon}(\tau), \quad j \in \mathcal{C} .
$$

Obviously, we have

$$
\left|u_{j}^{\varepsilon}(\tau)\right| \equiv\left|a_{j}^{\varepsilon}(\tau)\right| \quad \text { for all } j \in \mathcal{C}, \quad \text { and } \quad I\left(u^{\varepsilon}(\tau)\right) \equiv I\left(a^{\varepsilon}(\tau)\right) .
$$

The process $a^{\varepsilon}(\tau)$ satisfies

$$
\begin{aligned}
\dot{a}_{j}^{\varepsilon} & =\mathrm{e}^{-i \varepsilon^{-1} \tau} P_{j}\left(\mathrm{e}^{i \varepsilon^{-1} \tau} a^{\varepsilon}\right)+\sqrt{2 \mathcal{T}_{j}} \mathrm{e}^{-i \varepsilon^{-1} \tau} \dot{\beta}_{j}, \quad j \in \mathcal{C}, \\
\mathcal{D}\left(a^{\varepsilon}(0)\right) & =\mathcal{D}\left(u_{0}\right),
\end{aligned}
$$

where $\mathrm{e}^{i \varepsilon^{-1} \tau} a^{\varepsilon}$ is a vector with components $\left(\mathrm{e}^{i \varepsilon^{-1} \tau} a^{\varepsilon}\right)_{k}=\mathrm{e}^{i \varepsilon^{-1} \tau} a_{k}^{\varepsilon}, k \in \mathcal{C}$. Regarding (2.29) as an equation in the extended phase space $\mathbb{C}^{|\mathcal{C}|} \times \mathbb{R}_{+} \ni(a, \theta)$, we have

$$
\dot{a}_{j}^{\varepsilon}=\mathrm{e}^{-i \theta} P_{j}\left(\mathrm{e}^{i \theta} a^{\varepsilon}\right)+\sqrt{2 \mathcal{T}_{j}} \mathrm{e}^{-i \theta} \dot{\beta}_{j}, \quad \dot{\theta}=\varepsilon^{-1} .
$$

Equation (2.30) is a usual fast-slow system with the unique fast variable $\theta$. Applying the method of stochastic averaging due to R. Khas'minski, we will show that its limiting (as $\varepsilon \rightarrow 0$ ) dynamics is given by the $a$-equation, averaged with respect to $\theta$, see the next section. The latter has the form

$$
\dot{u}_{j}=\mathcal{R}_{j}(u)+\sqrt{\mathcal{T}_{j}} \dot{\boldsymbol{\beta}}_{j}, \quad j \in \mathcal{C},
$$

where

$$
\mathcal{R}_{j}(u):=\int_{0}^{2 \pi} \mathrm{e}^{-i \theta} P_{j}\left(\mathrm{e}^{i \theta} u\right) \mathrm{d} \theta, \quad \mathrm{d} \theta:=\frac{\mathrm{d} \theta}{2 \pi},
$$

11 Note that the function $P_{j}$ is real valued. 
and $\boldsymbol{\beta}=\left(\boldsymbol{\beta}_{j}\right)_{j \in \mathcal{C}}$ is a standard complex $|\mathcal{C}|$-dimensional Brownian motion, so that $\boldsymbol{\beta}_{j}=\beta_{j}^{1}+i \beta_{j}^{2}$, where $\left(\beta_{j}^{k}\right)_{k=1,2, j \in \mathcal{C}}$ are standard real independent Brownian motions. Following [29], we call (2.31) the effective equation.

Let us emphasize that under the limit $\varepsilon \rightarrow 0$ the noise "bifurcates". Indeed, the rank of the dispersion matrix of the effective equation written in the real $(p, q)$-coordinates equals to $2|\mathcal{C}|$ (so the diffusion matrix is nondegenerate), while that for Eq. (2.29) equals to $|\mathcal{C}|$. This happens because the fast rotation decomposes the noise into two independent components, see Proposition 4.3.

Let us calculate the drift $\mathcal{R}_{j}(u)$. For this purpose, we will need to define the resonant averaging. Take a continuous function $f: \mathbb{C}^{|\mathcal{C}|} \rightarrow \mathbb{R}$ and write it in the action-angle coordinates $(I, \varphi)$. Then, the resonant averaging $\langle f\rangle_{R}$ is defined as the averaging of $f$ in angles in the direction $\mathbf{1}=(1,1 \ldots, 1)$ of their fast rotation [see $\varphi$-equation in (1.8)]. More precisely,

$$
\langle f\rangle_{R}(I, \varphi):=\int_{0}^{2 \pi} f(I, \varphi+\theta \mathbf{1}) \mathrm{d} \theta .
$$

In the $u$-variables, it takes the form

$$
\langle f\rangle_{R}(u)=\int_{0}^{2 \pi} f\left(\mathrm{e}^{i \theta} u\right) \mathrm{d} \theta .
$$

Further on, we will often use properties of the resonant averaging given in Appendix C. Denote by $H^{\text {res }}$ the resonant averaging of the interaction potential,

$$
H^{\mathrm{res}}:=\frac{1}{2} \sum_{|m-k|=1} V_{m k}^{\mathrm{res}}, \quad \text { where } V_{m k}^{\mathrm{res}}:=\left\langle V_{m k}\right\rangle_{R} .
$$

We will call the function $V_{m k}^{\text {res }}$ the resonant potential and $H^{\text {res }}$ - the resonant Hamiltonian. In view of (2.32) and (2.26), we have

$$
\begin{aligned}
\mathcal{R}_{j}(u) & =\lambda \int_{0}^{2 \pi} \mathrm{e}^{-i \theta} \sum_{k:|k-j|=1} i \nabla_{j} V_{j k}\left(\mathrm{e}^{i \theta} u\right) \mathrm{d} \theta-\int_{0}^{2 \pi} \mathrm{e}^{-i \theta} \operatorname{Re}\left(\mathrm{e}^{i \theta} u_{j}\right) \mathrm{d} \theta \\
& =\lambda i \nabla_{j} \int_{0}^{2 \pi} \frac{1}{2} \sum_{|m-k|=1} V_{m k}\left(\mathrm{e}^{i \theta} u\right) \mathrm{d} \theta-\int_{0}^{2 \pi} \mathrm{e}^{-i \theta} \frac{\mathrm{e}^{i \theta} u_{j}+\mathrm{e}^{-i \theta} \bar{u}_{j}}{2} \mathrm{~d} \theta \\
& =\lambda i \nabla_{j} H^{\mathrm{res}}(u)-\frac{u_{j}}{2} .
\end{aligned}
$$

Then, the effective equation (2.31) takes the form

$$
\dot{u}_{j}=\lambda i \nabla_{j} H^{\mathrm{res}}-\frac{u_{j}}{2}+\sqrt{\mathcal{T}_{j}} \dot{\boldsymbol{\beta}}_{j}, \quad j \in \mathcal{C},
$$

and describes dynamics of a Hamiltonian system given by the resonant Hamiltonian $\lambda H^{\text {res }}$, where each particle is coupled with its own stochastic Langevintype thermostat. Note that Eq. (2.37), written in the real $(p, q)$-coordinates, coincides with Eq. (1.10) from the introduction. 


\subsection{Averaging Theorems}

It is known that Eq. (2.31) has a unique solution, this solution is defined globally (see [26]), and that Eq. (2.31) is mixing (see [26,44,45]). ${ }^{12}$ In Sect. 4.2, we will prove the following theorem, which jointly with (2.28) immediately implies Theorem 1.1.

Theorem 2.4. On the space $C\left([0, T], \mathbb{C}^{|\mathcal{C}|}\right)$, we have the weak convergence of measures

$$
\mathcal{D}\left(a^{\varepsilon}(\cdot)\right) \rightarrow \mathcal{D}(u(\cdot)) \quad \text { as } \varepsilon \rightarrow 0 \quad \text { uniformly in } \mathcal{C},
$$

where $u(\tau)$ is a unique solution of the effective equation (2.31) satisfying $\mathcal{D}(u(0))=\mathcal{D}\left(u_{0}\right)$.

Recall that the uniformity in $\mathcal{C}$ of the weak convergences of measures through all the text is understood in the sense of finite-dimensional projections. For example, for convergence (2.38), it means that for any bounded set $\Lambda \subset \mathbb{Z}^{d}$ and any continuous bounded functional $f: C\left([0, T], \mathbb{C}^{|\mathcal{C}|}\right) \mapsto \mathbb{R}$, satisfying ${ }^{13}$ Supp $f \subseteq \Lambda$, we have

$$
\mathbf{E} f\left(a^{\varepsilon}(\cdot)\right) \rightarrow \mathbf{E} f(u(\cdot)) \text { as } \varepsilon \rightarrow 0 \quad \text { uniformly in } \mathcal{C},
$$

where we assume that the set $\mathcal{C}$ satisfies $\mathcal{C} \supseteq \Lambda$.

Convergence (2.38) transfers the estimates for solutions of Eq. (1.6) obtained in Lemma 2.1 to the effective equation.

Proposition 2.5. Let $u(\tau)$ be the unique solution of the effective equation (2.31) satisfying $\mathcal{D}(u(0))=\mathcal{D}\left(u_{0}\right)$, and $\mu$ be its unique stationary measure. Then, for all $0<\lambda \leq 1, j \in \mathcal{C}$ and $\tau \geq 0$, we have

$$
\text { (i) } \mathbf{E} \max _{s \in[\tau, \tau+1]} \mathrm{e}^{\alpha\left|u_{j}(s)\right|^{2}} \leq C, \quad \text { (ii) }\left\langle\mu, \mathrm{e}^{\alpha\left|u_{j}\right|^{2}}\right\rangle \leq C,
$$

where the constants $C$ and $\alpha$ are the same that in Lemma 2.1.

Proof. Let $u^{\varepsilon}(\tau)$ be the unique solution of Eq. (2.25) satisfying $\mathcal{D}\left(u^{\varepsilon}(0)\right)=$ $\mathcal{D}\left(u_{0}\right)$. Writing the first estimate from Lemma 2.1 in the complex variables, we get $\mathbf{E} \max _{s \in[\tau, \tau+1]} \mathrm{e}^{\alpha\left|u_{j}^{\varepsilon}(s)\right|^{2}}<C$, for all $0<\varepsilon<\varepsilon_{0}, 0<\lambda \leq 1, j \in \mathcal{C}$ and $\tau \geq 0$. Then, (2.28) joined with Theorem 2.4 and the Fatou lemma implies item (i). Item (ii) follows from item (i), the mixing property of the effective equation and the Fatou lemma.

Next, we investigate the limiting (as $\varepsilon \rightarrow 0$ ) behaviour of the unique stationary measure $\mu^{\varepsilon}$ of Eq. (2.25). Let us introduce an additional assumption. Consider an unbounded set $\mathcal{C}_{\infty} \subseteq \mathbb{Z}^{d}$. Take the inductive limit as $\mathcal{C} \nearrow \mathcal{C}_{\infty}$ of the effective equation, i.e. Eq. $(2.31)$ with the set $\mathcal{C}$ replaced by $\mathcal{C}_{\infty}$. We will call it the $\mathcal{C}_{\infty}$-effective equation.

\footnotetext{
12 To establish the mixing property, one could also use the method explained in Appendix A.

13 Recall that Supp $f$ is defined in Agreements.6.
} 
Definition 2.6. $\quad$ (i) A weak solution $u^{\infty}(\tau)$ of the $\mathcal{C}_{\infty}$-effective equation is called regular if there exists $\Delta>0$ such that for any $T \geq 0$ we have

$$
\sup _{j \in \mathcal{C}_{\infty}} \mathbf{E} \max _{0 \leq \tau \leq T} \mathrm{e}^{\Delta\left|u_{j}^{\infty}(\tau)\right|^{2}}<\infty .
$$

(ii) A probability measure $\mu^{\infty}$ defined on the Borel $\sigma$-algebra $\mathcal{B}\left(\mathbb{C}^{\left|\mathcal{C}_{\infty}\right|}\right)^{14}$ is called regular stationary measure of the $\mathcal{C}_{\infty}$-effective equation if the latter admits a regular weak solution $u^{\infty}(\tau)$ satisfying $\mathcal{D}\left(u^{\infty}(\tau)\right) \equiv \mu^{\infty}$.

Sometimes, we will assume

$\mathbf{H} \mathcal{C}_{\infty}$. For each unbounded set $\mathcal{C}_{\infty} \subseteq \mathbb{Z}^{d}$, the corresponding $\mathcal{C}_{\infty}$-effective equation admits a unique regular stationary measure.

Passing to the limit $\mathcal{C} \nearrow \mathcal{C}_{\infty}$ and using estimates of Proposition 2.5, we can prove that the $\mathcal{C}_{\infty}$-effective equation has a regular stationary measure, but we do not know if it is unique (however, we believe that it is). The following result provides a sufficient condition for fulfillment of ass. $H \mathcal{C}_{\infty}$.

Proposition 2.7. Assume that $\lambda$ is sufficiently small and second partial derivatives of the resonant potentials $V_{k j}^{\text {res }}$ are bounded. ${ }^{15}$ Then, assumption $\mathrm{HC}_{\infty}$ is satisfied.

In particular, if the second partial derivatives of the interaction potential $V$ are bounded, then those of $V_{k j}^{\text {res }}$ also are. Proposition 2.7 is a corollary of Proposition 4.9, where we show that under conditions of Proposition 2.7 the $\mathcal{C}_{\infty}$-effective equation defines a mixing Markov process, in some appropriate space. Its proof is based on a simple observation that for $\lambda$ sufficiently small the $\mathcal{C}_{\infty}$-effective equation is contracting there. See Sect. 4.4 .

Theorem 2.8. (i) Let $\mu^{\varepsilon}$ and $\mu$ be the unique stationary measures of Eq. (2.25) and the effective equation (2.31) correspondingly. Then, $\mu^{\varepsilon} \rightarrow \mu$ as $\varepsilon \rightarrow 0$.

(ii) If assumption $\mathrm{HC}_{\infty}$ is satisfied, then the convergence above holds uniformly in $\mathcal{C}$.

The proof of the theorem is postponed to Sect. 4.3. It is based on a version of Theorem 2.4 for stationary solutions, and rotation invariance of the effective equation discussed below.

In Appendix A, it is proven that the rate of mixing of Eq. (2.25) is independent from $\varepsilon$. Jointly with Theorems 1.1 and 2.8, this implies the following result.

Theorem 2.9. (i) The convergence $\mathcal{D}\left(I\left(u^{\varepsilon}(\tau)\right)\right) \rightarrow \mathcal{D}(I(u(\tau)))$ as $\varepsilon \rightarrow 0$ provided by Theorem 1.1 holds uniformly in $\tau \geq 0$ in the sense that for any continuous bounded function $f: \mathbb{R}^{|\mathcal{C}|} \mapsto \mathbb{R}$ we have

\footnotetext{
14 The space $\mathbb{C}^{\left|\mathcal{C}_{\infty}\right|}$ is provided with the Tikhonov topology of pointwise convergence.

15 If the second partial derivatives of the function $V_{k j}^{\text {res }}$ are bounded for one pair of indices $k, j \in \mathcal{C}$, then they are bounded by the same constant for all other $k, j \in \mathcal{C}$. Indeed, this is evident since $V_{k j}^{\text {res }}(u)=\left\langle V\left(q_{k}(u), q_{j}(u)\right)\right\rangle_{R}$.
} 


$$
\sup _{\tau \geq 0}\left|\mathbf{E} f\left(I^{\varepsilon}(\tau)\right)-\mathbf{E} f(I(\tau))\right| \rightarrow 0 \quad \text { as } \quad \varepsilon \rightarrow 0
$$

where $I^{\varepsilon}(\tau):=I\left(u^{\varepsilon}(\tau)\right)$ and $I(\tau):=I(u(\tau)) .{ }^{16}$

(ii) We have

$$
\lim _{\varepsilon \rightarrow 0} \lim _{\tau \rightarrow \infty} \mathcal{D}\left(I^{\varepsilon}(\tau)\right)=\lim _{\tau \rightarrow \infty} \lim _{\varepsilon \rightarrow 0} \mathcal{D}\left(I^{\varepsilon}(\tau)\right)=\lim _{\substack{\varepsilon \rightarrow 0 \\ \tau \rightarrow \infty}} \mathcal{D}\left(I^{\varepsilon}(\tau)\right)=\Pi_{I *} \mu,
$$

where $\mu$ is the unique stationary measure of the effective equation (2.31), and $\Pi_{I}$ denotes the projection to the space of actions.

Proof. (i) Equations (2.25) and (2.31) are mixing and the rate of mixing for Eq. (2.25) is uniform in $\varepsilon<\varepsilon_{1}$ (see Theorem A.1). Then, for any $\delta>0$, there exists $s>0$ such that for any $\varepsilon$ satisfying $\varepsilon<\varepsilon_{1}$

$$
\sup _{\tau \geq s}\left|\mathbf{E} f\left(I^{\varepsilon}(\tau)\right)-\left\langle\Pi_{I *} \mu^{\varepsilon}, f\right\rangle\right| \leq \delta / 3, \quad \sup _{\tau \geq s}\left|\mathbf{E} f(I(\tau))-\left\langle\Pi_{I *} \mu, f\right\rangle\right| \leq \delta / 3,
$$

where $\mu^{\varepsilon}$ is the unique stationary measure of Eq. (2.25). In view of Theorem 2.8.i, there exists $\varepsilon_{2}$ such that for any $\varepsilon<\varepsilon_{2}$ we have

$$
\left|\left\langle\Pi_{I *} \mu^{\varepsilon}, f\right\rangle-\left\langle\Pi_{I *} \mu, f\right\rangle\right| \leq \delta / 3 \text {. }
$$

Inequalities (2.40) and (2.41) imply that for $\varepsilon<\varepsilon_{1} \wedge \varepsilon_{2}$ we have

$$
\sup _{\tau \geq s}\left|\mathbf{E} f\left(I^{\varepsilon}(\tau)\right)-\mathbf{E} f(I(\tau))\right| \leq \delta .
$$

Applying Theorem 1.1 and using that the weak convergence of measures is equivalent to convergence in the dual-Lischitz norm (see [17], Theorem 11.3.3), it is not difficult to show that $\sup _{0 \leq \tau \leq s}\left|\mathbf{E} f\left(I^{\varepsilon}(\tau)\right)-\mathbf{E} f(I(\tau))\right| \rightarrow 0$ as $\varepsilon \rightarrow 0$. Then, there exists $\varepsilon_{3}$, such that for any $\varepsilon<\varepsilon_{3}$ we have $\sup _{0 \leq \tau \leq s} \mid \mathbf{E} f\left(I^{\varepsilon}(\tau)\right)$ $-\mathbf{E} f(I(\tau)) \mid \leq \delta$. Jointly with (2.42), this implies that for $\varepsilon<\varepsilon_{1} \wedge \varepsilon_{2} \wedge \varepsilon_{3}$ we have $\sup _{\tau \geq 0}\left|\mathbf{E} f\left(I^{\varepsilon}(\tau)\right)-\mathbf{E} f(I(\tau))\right| \leq \delta$.

(ii) The first two convergences follow from the mixing properties of Eqs. (2.25) and (2.31), and Theorems 1.1, 2.8.i. The last convergence follows from item (i) joined with the mixing property of Eq. (2.31).

\subsection{The Limiting Behaviour of the Local Energy}

Recall that the local energy $\mathcal{E}_{j}^{\nu}(p, q)$ is defined in (1.12). We will consider it as a function of the complex variables, and abusing notations we will write $\mathcal{E}_{j}^{\nu}(u)$. Define the vector of local energy

$$
\mathcal{E}^{\nu}(u):=\left(\mathcal{E}_{j}^{\nu}(u)\right)_{j \in \mathcal{C}} .
$$

Let $u^{\varepsilon}(\tau)$ be a solution of Eq. (2.25), either satisfying $\mathcal{D}\left(u^{\varepsilon}(0)\right)=\mathcal{D}\left(u_{0}\right)$ or a stationary one. Since $\mathcal{E}^{\nu}=I+O(\nu)$, the limiting as $\varepsilon \rightarrow 0$ behaviour of the vector of local energy $\mathcal{E}^{\nu}\left(u^{\varepsilon}\right)$ coincides with that of the vector of actions $I\left(u^{\varepsilon}\right)$, while the latter is governed by the effective equation, in the sense of Theorems 1.1, 2.8 and 2.9. More precisely, we prove

\footnotetext{
${ }^{16}$ We do not know if the convergence holds uniformly in both $\mathcal{C}$ and $\tau$.
} 
Proposition 2.10. Let $u^{\varepsilon}(\tau)$ be a solution of Eq. (2.25) satisfying $\mathcal{D}(u(0))=$ $\mathcal{D}\left(u_{0}\right)$, and $\widetilde{u}^{\varepsilon}(\tau)$ be its stationary solution. Let $u(\tau)$ be a solution of the effective equation (2.31) satisfying $\mathcal{D}(u(0))=\mathcal{D}\left(u_{0}\right)$, and $\mu$ be its unique stationary measure. Then, denoting $\mathcal{E}^{\nu}(\tau):=\mathcal{E}^{\nu}\left(u^{\varepsilon}(\tau)\right), \widetilde{\mathcal{E}}^{\nu}(\tau):=\mathcal{E}^{\nu}\left(\widetilde{u}^{\varepsilon}(\tau)\right)$ and $I(\tau):=I(u(\tau))$, we have

(i) $\mathcal{D}\left(\mathcal{E}^{\nu}(\cdot)\right) \rightarrow \mathcal{D}(I(\cdot))$ as $\varepsilon \rightarrow 0$ on $C\left([0, T], \mathbb{R}^{|\mathcal{C}|}\right)$ uniformly in $\mathcal{C}$.

(ii) $\mathcal{D}\left(\mathcal{E}^{\nu}(\tau)\right) \rightarrow \mathcal{D}(I(\tau))$ as $\varepsilon \rightarrow 0$ uniformly in $\tau \geq 0$ in the sense explained in Theorem 2.9 .

(iii) $\mathcal{D}\left(\widetilde{\mathcal{E}}^{\nu}(\tau)\right) \rightarrow \Pi_{I *} \mu$ as $\varepsilon \rightarrow 0$ for any $\tau \geq 0$. If ass. $H \mathcal{C}_{\infty}$ is satisfied, this convergence holds uniformly in $\mathcal{C}$.

(iv) $\lim _{\varepsilon \rightarrow 0} \lim _{\tau \rightarrow \infty} \mathcal{D}\left(\mathcal{E}^{\nu}(\tau)\right)=\lim _{\tau \rightarrow \infty} \lim _{\mathcal{E} \rightarrow 0} \mathcal{D}\left(\mathcal{E}^{\nu}(\tau)\right)=\lim _{\substack{\varepsilon \rightarrow 0 \\ \tau \rightarrow \infty}}$ $\mathcal{D}\left(\mathcal{E}^{\nu}(\tau)\right)=\Pi_{I *} \mu$.

Proof. Since the interaction potential $V$ has at most a polynomial growth, the estimate (i) of Lemma 2.1 implies that the limit as $\varepsilon \rightarrow 0$ of $\mathcal{D}\left(\mathcal{E}^{\nu}(\cdot)\right)$ coincides with that of $\mathcal{D}\left(I\left(u^{\varepsilon}(\cdot)\right)\right)$ in the sense that if one limit exists, then another one exists as well and the two are equal. Moreover, if one convergence is uniform in $\mathcal{C}$, then another one also is. That is why item (i) follows from Theorem 1.1. Items (ii)-(iv) can be obtained similarly, using Theorems 2.8, 2.9.i, ii correspondingly. For the first limit from item (iv), one should additionally use that Eq. (2.25) is mixing.

\subsection{Rotation Invariance of the Effective Equation}

Further on, we will need the following results. Since the effective equation (2.31) is obtained by the resonant averaging procedure, it is rotation invariant in the direction $\mathbf{1}$ of the fast rotation of angles:

Proposition 2.11. If $u(\tau)$ is a weak solution of the effective equation (2.31), then for any $\xi \in[0,2 \pi)$ the process $\mathrm{e}^{i \xi} u(\tau)$ is also its weak solution.

Proof. Process $w(\tau)=\mathrm{e}^{i \xi} u(\tau)$ is a weak solution of equation

$$
\dot{w}_{j}=\int_{0}^{2 \pi} \mathrm{e}^{-i(\theta-\xi)} P_{j}\left(\mathrm{e}^{i \theta} u\right) \mathrm{d} \theta+\sqrt{\mathcal{T}_{j}} \mathrm{e}^{i \xi} \dot{\boldsymbol{\beta}}_{j}, \quad j \in \mathcal{C} .
$$

Changing the variable of integration $\widetilde{\theta}:=\theta-\xi$ and noting that $\left\{\mathrm{e}^{i \xi} \boldsymbol{\beta}_{j}, j \in \mathcal{C}\right\}$ is another set of standard complex-independent Brownian motions, we obtain that the process $w(\tau)$ is a weak solution of the effective equation (2.31).

Proposition 2.12. Let $\mu$ be the unique stationary measure of the effective equation (2.31). Then, for any continuous function $f: \mathbb{C}^{|\mathcal{C}|} \mapsto \mathbb{R}$ with at most a polynomial growth at infinity, we have

$$
\langle\mu, f\rangle=\left\langle\mu,\langle f\rangle_{R}\right\rangle .
$$


Proof. Since the stationary measure $\mu$ of the effective equation is unique, Proposition 2.11 implies that it is rotation invariant in the direction 1: for any $\xi \in[0,2 \pi)$ we have $\left(\mathrm{e}^{i \xi}\right)_{*} \mu=\mu$. Then

$$
\langle\mu, f\rangle=\int_{0}^{2 \pi}\left\langle\left(\mathrm{e}^{i \xi}\right)_{*} \mu, f\right\rangle \mathrm{d} \xi=\left\langle\mu, \int_{0}^{2 \pi} f\left(\mathrm{e}^{i \xi} \cdot\right) \mathrm{d} \xi\right\rangle=\left\langle\mu,\langle f\rangle_{R}\right\rangle .
$$

\section{The Energy Transport}

In this section, we assume that $\lambda \ll 1$ and investigate the limiting (as $\varepsilon \rightarrow 0$ ) behaviour of the stationary Hamiltonian energy flow for Eq. (2.25). We prove Theorems 3.4 and 3.12 which are full versions of Theorems 1.6 and 1.8 given in the introduction.

\subsection{The Limiting Behaviour of the Energy Flow}

Consider the Ornstein-Uhlenbeck process $\left.(2.37)\right|_{\lambda=0}$

$$
\dot{u}_{m}=-\frac{u_{m}}{2}+\sqrt{\mathcal{T}_{m}} \dot{\boldsymbol{\beta}}_{m}, \quad m \in \mathcal{C} .
$$

It is well known that the unique stationary measure $\mu^{0}$ of Eq. (3.1) has the form

$$
\mu^{0}=\mathrm{e}^{-|u|_{\mathcal{T}}^{2}} d m
$$

where $|u|_{\mathcal{T}}^{2}:=\sum_{m \in \mathcal{C}}\left(2 \mathcal{T}_{m}\right)^{-1}\left|u_{m}\right|^{2}$ and $d m$ is a normalized Lebesgue measure on $\mathbb{C}^{|\mathcal{C}|} \simeq \mathbb{R}^{2|\mathcal{C}|}, d m=d$ Leb $/\left((2 \pi)^{|\mathcal{C}|} \Pi_{m \in \mathcal{C}} \mathcal{T}_{m}\right)$. The Ornstein-Uhlenbeck process (3.1) is important for our study because of the following

Proposition 3.1. (i) Let $u^{\lambda}(\tau)$ and $u(\tau)$ be solutions of the effective equation (2.37) and Eq. (3.1) correspondingly, satisfying $\mathcal{D}\left(u^{\lambda}(0)\right)=\mathcal{D}(u(0))=$ $\mathcal{D}\left(u_{0}\right)$. Then

$$
\mathcal{D}\left(u^{\lambda}(\cdot)\right) \rightarrow \mathcal{D}(u(\cdot)) \quad \text { as } \quad \lambda \rightarrow 0 \quad \text { on } \quad C\left([0, T], \mathbb{C}^{|\mathcal{C}|}\right) \quad \text { uniformly in } \mathcal{C} .
$$

(ii) Let $\mu^{\lambda}$ be the unique stationary measure of Eq. (2.37). Then, $\mu^{\lambda} \rightarrow \mu^{0}$ as $\lambda \rightarrow 0$ uniformly in $\mathcal{C}$.

Proof. The required convergences can be established by arguments similar to those used in the proofs of Theorems 2.4 and 2.8, but significantly simplified. The uniformity of convergence from item (ii), similarly to that of Theorem 2.8.ii, follows from fulfillment of assumption analogous to $H \mathcal{C}_{\infty}$. Namely, since Eq. (3.1) is diagonal, for any unbounded set $\mathcal{C}_{\infty} \subseteq \mathbb{Z}^{d}$ a stationary measure of its inductive limit as $\mathcal{C} \nearrow \mathcal{C}_{\infty}$ [given by $(3.1)$ with $\mathcal{C}=\mathcal{C}_{\infty}$ ] is unique. 
For $k, j \in \mathbb{Z}^{d}$ denote by $A^{k j}$ the generator of the process (3.1) with $\mathcal{C}$ replaced by the set $\{k, j\}$. Considering $A^{k j}$ as an operator on real valued functions, we have

$$
A^{k j}=\sum_{l=j, k}\left(2 \mathcal{T}_{l} \partial_{u_{l} \bar{u}_{l}}^{2}-\frac{u_{l}}{2} \cdot \nabla_{l}\right) .
$$

We will need the following well-known property of the Ornstein-Uhlenbeck process.

Lemma 3.2. (i) Fix $k, j \in \mathcal{C}$. Let a measurable function $\xi: \mathbb{C}^{|\mathcal{C}|} \mapsto \mathbb{R}$ has at most a polynomial growth at infinity, and satisfies $\operatorname{Supp} \xi \subseteq\{k, j\}$ and $\left\langle\mu^{0}, \xi\right\rangle=0$. Then, equation

$$
A^{k j} \eta=\xi
$$

has a unique solution $\eta: \mathbb{C}^{|\mathcal{C}|} \mapsto \mathbb{R}$ in a class of functions satisfying the following properties (a),(b), (c): (a) $\eta$ belongs to each Sobolev class $W_{p, l o c}^{2}, p>1$, (b) $\eta$ has at most a polynomial growth at infinity, and (c) $\left\langle\mu^{0}, \eta\right\rangle=0$. The solution $\eta$ satisfies $\operatorname{Supp} \eta \subseteq\{j, k\}$ and has the form

$$
\eta(u)=-\int_{0}^{\infty} \mathfrak{P}_{\tau}^{0} \xi(u) \mathrm{d} \tau,
$$

where $\mathfrak{P}_{\tau}^{0}$ denotes a Markov semigroup associated with the OrnsteinUhlenbeck process (3.1). Moreover, the gradient $\nabla \eta(u)$ also has at most a polynomial growth at infinity.

(ii) If the function $\xi$ is $C^{n}$-smooth for some $n \in \mathbb{N}$, then the solution $\eta$ is also $C^{n}$-smooth.

Proof. (i) This is a particular case of Theorem 1 from [37].

(ii) It follows in a standard way from smoothness of coefficients of the generator $A^{k j}, C^{n}$-smoothness of the function $\xi$ and the Sobolev embedding theorem (see [42], Theorem 5.11.1).

Recall that the energy flow $\mathcal{J}_{k j}(p, q)$ is defined in (1.14). Abusing notations, we put $\mathcal{J}_{k j}(u):=\mathcal{J}_{k j}(p(u), q(u))$. Introduce the resonant energy flow

$$
\mathcal{J}_{k j}^{\mathrm{res}}:=\left\langle\mathcal{J}_{k j}\right\rangle_{R} \text {. }
$$

Proposition C.1.ii joined with ass. $H V$ implies that the function $\mathcal{J}_{k j}^{\text {res }}$ is $C^{r-1}$ smooth, and all its partial derivatives have at most a polynomial growth at infinity.

Proposition 3.3. We have

$$
\text { (i) } \mathcal{J}_{k j}^{\text {res }}=2 \partial_{\varphi_{k}} V_{k j}^{\text {res }}=-2 \partial_{\varphi_{j}} V_{k j}^{\text {res }} ; \quad \text { (ii) }\left\langle\mu^{0}, \mathcal{J}_{k j}^{\text {res }}\right\rangle=0 .
$$

Proof. (i). The Poisson bracket written in the action angle coordinates $(I, \varphi)$ takes the form $\left\{h_{1}, h_{2}\right\}=\sum_{k \in \mathcal{C}}\left(\partial_{\varphi_{k}} h_{1} \partial_{I_{k}} h_{2}-\partial_{I_{k}} h_{1} \partial_{\varphi_{k}} h_{2}\right)$. Then, (1.14) implies

$$
\mathcal{J}_{k j}=\left\{I_{j}-I_{k}, V_{k j}\right\}=\left(\partial_{\varphi_{k}} V_{k j}-\partial_{\varphi_{j}} V_{k j}\right)
$$


Proposition C.1.iii provides that for $l \in\{j, k\}$ we have $\left\langle\partial_{\varphi_{l}} V_{k j}\right\rangle_{R}$ $=\partial_{\varphi_{l}}\left\langle V_{k j}\right\rangle_{R}=\partial_{\varphi_{l}} V_{k j}^{\text {res }}$. It remains to take the resonant averaging of (3.6) and note that Proposition C.1.iv implies

$$
\partial_{\varphi_{k}} V_{k j}^{\mathrm{res}}=-\partial_{\varphi_{j}} V_{k j}^{\mathrm{res}}
$$

(ii) It follows from item (i) and rotation invariance of the Gaussian measure $\mu^{0}$.

In view of item (ii) of Proposition 3.3, the resonant energy flow $\mathcal{J}_{k j}^{\text {res }}$ satisfies conditions of Lemma 3.2. Denote by $\eta_{k j}(u)$ obtained there solution of equation

$$
A^{k j} \eta_{k j}=\mathcal{J}_{k j}^{\mathrm{res}}
$$

Theorem 3.4. Let $\mu^{\varepsilon, \lambda}$ be the unique stationary measure of Eq. (2.25). Then, for every $j, k \in \mathcal{C},|j-k|=1$, we have

$$
\left\langle\mu^{\varepsilon, \lambda}, \mathcal{J}_{k j}\right\rangle \rightarrow \lambda \kappa\left(\mathcal{T}_{k}, \mathcal{T}_{j}\right)\left(\mathcal{T}_{k}-\mathcal{T}_{j}\right)+o(\lambda) \quad \text { as } \quad \varepsilon \rightarrow 0
$$

where $o(\lambda) / \lambda \rightarrow 0$ as $\lambda \rightarrow 0$ uniformly in $\mathcal{C}$, and

$$
\kappa\left(\mathcal{T}_{k}, \mathcal{T}_{j}\right)=-\frac{\left\langle\mu^{0}, \mathcal{J}_{k j}^{\mathrm{res}} \eta_{k j}\right\rangle}{\mathcal{T}_{k} \mathcal{T}_{j}}=\frac{\left\langle\mu^{0}, \mathcal{T}_{k}\left|\nabla_{k} \eta_{k j}\right|^{2}+\mathcal{T}_{j}\left|\nabla_{j} \eta_{k j}\right|^{2}\right\rangle}{2 \mathcal{T}_{k} \mathcal{T}_{j}} .
$$

If assumption $\mathrm{HC}_{\infty}$ is satisfied, then convergence (3.9) holds uniformly in $\mathcal{C}$.

The function $\kappa: \mathbb{R}_{+}^{2} \mapsto \mathbb{R}$ satisfies $\kappa(x, y) \equiv \kappa(y, x)$, is $C^{r-1}$-smooth and nonnegative. If the resonant potential $V_{k j}^{\text {res }}$ is not independent from the angles $\varphi$, then $\kappa$ is strictly positive.

Emphasize that the function $\kappa\left(\mathcal{T}_{k}, \mathcal{T}_{j}\right)$, which we call the conductivity, implicitly depends on the temperatures through the measure $\mu^{0}$ and the function $\eta_{k j}$ (the latter depends on them since the operator $A^{k j}$ does).

Since $\eta_{k j}$ is the solution of Eq. (3.8) obtained in Lemma 3.2, formula (3.5) joined with (3.10) implies that the conductivity $\kappa$ can be also represented in the form

$$
\kappa\left(\mathcal{T}_{k}, \mathcal{T}_{j}\right)=\frac{1}{\mathcal{T}_{k} \mathcal{T}_{j}}\left\langle\mu^{0}, \mathcal{J}_{k j}^{\text {res }} \int_{0}^{\infty} \mathfrak{P}_{\tau}^{0} \mathcal{J}_{k j}^{\text {res }} \mathrm{d} \tau\right\rangle .
$$

Note that the sufficient condition for fulfillment of ass. $\mathrm{HC}_{\infty}$ provided by Proposition 2.7 is relevant for Theorem 3.4, since convergence (3.9) gives some information only if $\lambda$ is small.

To prove Theorem 3.4, we will need the following proposition, which can be obtained by simple integration by parts.

Proposition 3.5. Let functions $\eta^{1}, \eta^{2} \in W_{2, \text { loc }}^{2}\left(\mathbb{C}^{|\mathcal{C}|}, \mathbb{R}\right)$ with their gradients $\nabla \eta^{1}, \nabla \eta^{2}$ have at most a polynomial growth at infinity. Then

$$
\left\langle\mu^{0}, \eta^{2} A^{k j} \eta^{1}\right\rangle=-\frac{1}{2}\left\langle\mu^{0}, \sum_{l=k, j} \mathcal{T}_{l} \nabla_{l} \eta^{1} \cdot \nabla_{l} \eta^{2}\right\rangle=\left\langle\mu^{0}, \eta^{1} A^{k j} \eta^{2}\right\rangle .
$$


Proof of Theorem 3.4. Denote by $\mu^{\lambda}$ the unique stationary measure of the effective equation (2.37). Theorem 2.8.i implies

$$
\left\langle\mu^{\varepsilon, \lambda}, \mathcal{J}_{k j}\right\rangle \rightarrow\left\langle\mu^{\lambda}, \mathcal{J}_{k j}\right\rangle=\left\langle\mu^{\lambda}, \mathcal{J}_{k j}^{\text {res }}\right\rangle \quad \text { as } \quad \varepsilon \rightarrow 0,
$$

where the last equality follows from Proposition 2.12. If ass. $H \mathcal{C}_{\infty}$ is satisfied, then Theorem 2.8.ii implies that convergence (3.11) holds uniformly in $\mathcal{C}$. Let us show that

$$
\left\langle\mu^{\lambda}, \mathcal{J}_{k j}^{\mathrm{res}}\right\rangle=\lambda \kappa\left(\mathcal{T}_{k}, \mathcal{T}_{j}\right)\left(\mathcal{T}_{k}-\mathcal{T}_{j}\right)+o(\lambda)
$$

where $o(\lambda) / \lambda \rightarrow 0$ as $\lambda \rightarrow 0$ uniformly in $\mathcal{C}$. The key role will play the following:

Lemma 3.6. Let the function $\xi \in C^{1}\left(\mathbb{C}^{|\mathcal{C}|}, \mathbb{R}\right)$ satisfies $\operatorname{Supp} \xi \subseteq\{k, j\}$, has at most a polynomial growth at infinity and $\left\langle\mu^{0}, \xi\right\rangle=0$. Then

$$
\left\langle\mu^{\lambda}, \xi\right\rangle=\lambda\left\langle\mu^{0}, f \xi\right\rangle+o(\lambda),
$$

where $o(\lambda) / \lambda \rightarrow 0$ as $\lambda \rightarrow 0$ uniformly in $\mathcal{C}, f=\frac{1}{2} \sum_{|m-n|=1} f_{m n}$, where $f_{m n}=f_{n m}$, and $f_{m n}$ is the solution of equation

$$
A^{m n} f_{m n}=\frac{\mathcal{T}_{n}-\mathcal{T}_{m}}{\mathcal{T}_{m} \mathcal{T}_{n}} \mathcal{J}_{m n}^{\mathrm{res}}
$$

obtained in Lemma $3.2 .^{17}$

Before proving the lemma, we will finish the proof of the theorem. Applying Lemma 3.6 to the function $\xi=\mathcal{J}_{k j}^{\text {res }}$, we get

$$
\left\langle\mu^{\lambda}, \mathcal{J}_{k j}^{\mathrm{res}}\right\rangle=\frac{\lambda}{2} \sum_{|m-n|=1}\left\langle\mu^{0}, f_{m n} \mathcal{J}_{k j}^{\mathrm{res}}\right\rangle+o(\lambda) .
$$

Proposition 3.7. If the sets $\{m, n\}$ and $\{k, j\}$ do not coincide, then

$$
\left\langle\mu^{0}, f_{m n} \mathcal{J}_{k j}^{\text {res }}\right\rangle=0 \text {. }
$$

Proof. Since $\operatorname{Supp} f_{m n} \subseteq\{m, n\}$, if $m, n \neq k$ or $m, n \neq j$ then the function $f_{m n}$ does not depend on the angle $\varphi_{k}$ or $\varphi_{j}$ correspondingly. Then, (3.16) follows from Proposition 3.3.i and rotation invariance of the Gaussian measure $\mu^{0}$.

Since $f_{j k}=f_{k j},(3.15)$ joined with Proposition 3.7 implies

$$
\left\langle\mu^{\lambda}, \mathcal{J}_{k j}^{\text {res }}\right\rangle=\lambda\left\langle\mu^{0}, f_{k j} \mathcal{J}_{k j}^{\text {res }}\right\rangle+o(\lambda) .
$$

Let $\eta_{k j}$ be the solution of Eq. (3.8), obtained in Lemma 3.2. Equation (3.14) implies

$$
f_{k j}=\frac{\mathcal{T}_{j}-\mathcal{T}_{k}}{\mathcal{T}_{k} \mathcal{T}_{j}} \eta_{k j}, \quad \text { so that } \quad\left\langle\mu^{0}, f_{k j} \mathcal{J}_{k j}^{\mathrm{res}}\right\rangle=\hat{\kappa}_{k j}(\mathcal{T})\left(\mathcal{T}_{k}-\mathcal{T}_{j}\right),
$$

where by $\mathcal{T}$ we have denoted the temperature profile $\left(\mathcal{T}_{j}\right)_{j \in \mathcal{C}}$, and $\hat{\kappa}_{k j}(\mathcal{T}):=-\frac{\left\langle\mu^{0}, \eta_{k j} \mathcal{J}_{k j}^{\text {res }}\right\rangle}{\mathcal{T}_{k} \mathcal{T}_{j}}$. Using that the function $\mathcal{J}_{k j}^{\text {res }}$ does not depend on

17 Fix a bounded set $\Lambda \subset \mathbb{Z}^{d}$. Almost without changes in the proof, a similar result can be established for a function $\xi$ satisfying $\operatorname{Supp} \xi \subseteq \Lambda$. We restrict ourselves to the case $\Lambda=\{k, j\}$, since we will apply the lemma only for a two-dimensional situation. 
the temperatures $\mathcal{T}, \eta_{k j}$ depends on them only through the components $\mathcal{T}_{k}, \mathcal{T}_{j}$, that Supp $\mathcal{J}_{k j}^{\text {res }}$, Supp $\eta_{k j} \subseteq\{j, k\}$ and $\mu^{0}$ is a product measure, we see that the function $\hat{\kappa}_{k j}$ depends on $\mathcal{T}$ only through $\mathcal{T}_{k}, \mathcal{T}_{j}$, so that $\hat{\kappa}_{k j}(\mathcal{T})=\kappa_{k j}\left(\mathcal{T}_{k}, \mathcal{T}_{j}\right)$, for some function $\kappa_{k j}$. Obviously, the latter does not depend on the choice of the indices $k, j$, so we skip them and get

$$
\kappa\left(\mathcal{T}_{k}, \mathcal{T}_{j}\right)=-\frac{\left\langle\mu^{0}, \eta_{k j} \mathcal{J}_{k j}^{\mathrm{res}}\right\rangle}{\mathcal{T}_{k} \mathcal{T}_{j}}
$$

Now (3.18) joined with (3.17) and (3.11) implies the desired convergence (3.9). To obtain the second representation from (3.10) for the conductivity $\kappa$, first we use Eq. (3.8) and then Proposition 3.5:

$$
\left\langle\mu^{0}, \eta_{k j} \mathcal{J}_{k j}^{\mathrm{res}}\right\rangle=\left\langle\mu^{0}, \eta_{k j} A^{k j} \eta_{k j}\right\rangle=-\frac{1}{2}\left\langle\mu^{0}, \sum_{l=k, j} \mathcal{T}_{l}\left|\nabla_{l} \eta_{k j}\right|^{2}\right\rangle,
$$

so that we get $\kappa\left(\mathcal{T}_{k}, \mathcal{T}_{j}\right)=\frac{\left\langle\mu_{0}, \sum_{l=k, j} \mathcal{T}_{l}\left|\nabla_{l} \eta_{k j}\right|^{2}\right\rangle}{2 \mathcal{T}_{k} \mathcal{T}_{j}}$. In particular, this implies that the conductivity $\kappa$ is nonnegative. Moreover, if the resonant potential $V_{k j}^{\text {res }}$ is not independent from the angles $\varphi$, then in view of Proposition 3.3.i the function $\mathcal{J}_{k j}^{\text {res }}$ does not vanish. Consequently, $\eta_{k j}$ is not a constant, so $\kappa$ is strictly positive for any $\mathcal{T}_{k}, \mathcal{T}_{j}>0$.

Let us show that $\kappa\left(\mathcal{T}_{k}, \mathcal{T}_{j}\right)=\kappa\left(\mathcal{T}_{j}, \mathcal{T}_{k}\right)$. Since $\mathcal{J}_{k j}^{\text {res }}=-\mathcal{J}_{j k}^{\text {res }}$ and $A^{k j}=$ $A^{j k}$, we have $\eta_{k j}=-\eta_{j k}$. Then, due to (3.19),

$$
\kappa\left(\mathcal{T}_{k}, \mathcal{T}_{j}\right)=-\left(\mathcal{T}_{k} \mathcal{T}_{j}\right)^{-1}\left\langle\mu^{0}, \eta_{j k} \mathcal{J}_{j k}^{\text {res }}\right\rangle=\kappa\left(\mathcal{T}_{j}, \mathcal{T}_{k}\right)
$$

It remains to prove that the function $\kappa$ is $C^{r-1}$-smooth. It is convenient to change the variables: let $v_{j}=\mathcal{T}_{j}^{-1 / 2} u_{j}, j \in \mathcal{C}$. Then, due to (3.19), we have

$$
\kappa\left(\mathcal{T}_{k}, \mathcal{T}_{j}\right)=-\left(\mathcal{T}_{k} \mathcal{T}_{j}\right)^{-1}\left\langle\widetilde{\mu}^{0}, \widetilde{\mathcal{J}}_{k j}^{\mathrm{res}} \widetilde{\eta}_{k j}\right\rangle
$$

where $\widetilde{\mu}^{0}:=\mathrm{e}^{-|v|^{2} / 2^{|\mathcal{C}|}} \mathrm{d} \widetilde{m}, \mathrm{~d} \widetilde{m}:=\mathrm{dLeb} /(2 \pi)^{|\mathcal{C}|}, \widetilde{\mathcal{J}}_{k j}^{\text {res }}(v):=\mathcal{J}_{k j}^{\text {res }}(u(v))$ and $\widetilde{\eta}_{k j}(v):=\eta_{k j}(u(v))$.

Proposition 3.8. For every $v \in \mathbb{C}^{|\mathcal{C}|}$, the functions $\widetilde{\mathcal{J}}_{k j}^{\text {res }}(v)=\widetilde{\mathcal{J}}_{k j}^{\text {res }}\left(v, \mathcal{T}_{k}, \mathcal{T}_{j}\right)$ and $\widetilde{\eta}_{k j}(v)=\widetilde{\eta}_{k j}\left(v, \mathcal{T}_{k}, \mathcal{T}_{j}\right)$ are $C^{r-1}$-smooth with respect to the temperatures $\left(\mathcal{T}_{k}, \mathcal{T}_{j}\right) \in \mathbb{R}_{+}^{2}$. The corresponding partial derivatives till the order $r-1$ inclusive are continuous in $\left(v, \mathcal{T}_{k}, \mathcal{T}_{j}\right) \in \mathbb{C}^{|\mathcal{C}|} \times \mathbb{R}_{+}^{2}$ and have at most a polynomial growth at infinity in $v$, which is uniform with respect to the temperatures $\left(\mathcal{T}_{k}, \mathcal{T}_{j}\right)$ if they belong to a compact set in $\mathbb{R}_{+}^{2}$.

Since the function $\mathcal{J}_{k j}^{\text {res }}$ is independent from the temperatures $\mathcal{T}_{k}, \mathcal{T}_{j}$, assertion of Proposition 3.8 concerning the function $\widetilde{\mathcal{J}}_{k j}^{\text {res }}$ is obvious. That, concerning $\widetilde{\eta}_{k j}$ is not so clear. It is not implied by standard arguments of elliptic theory because the generator $A^{k j}$ has growing coefficients. Its proof is based on simple analysis of formula (3.5) and is postponed to the end of the section. 
Since the measure $\widetilde{\mu}^{0}$ has an exponentially decaying density, Proposition 3.8 implies that the function $\left\langle\widetilde{\mu}^{0}, \widetilde{\mathcal{J}}_{k j}^{\text {res }} \widetilde{\eta}_{k j}\right\rangle$ is $C^{r-1}$-smooth, so that the conductivity $\kappa$ also is.

Proof of Lemma 3.6. It is known (see [9]) that the unique stationary measure $\mu^{\lambda}$ of the effective equation $(2.37)$ has a continuous density $\rho^{\lambda}$ with respect to the normalized Lebesgue measure $d m$, so that $d \mu^{\lambda}=\rho^{\lambda} d m$.

Then, in view of (3.2), we have

$$
\mathrm{d} \mu^{\lambda}=h^{\lambda} \mathrm{d} \mu^{0}
$$

where the density $h^{\lambda}$ satisfies

$$
\rho^{\lambda}=\mathrm{e}^{-|u|_{\mathcal{T}}^{2}} h^{\lambda} .
$$

The proof of the lemma is based on a decomposition of the density $h^{\lambda}$ in the small parameter $\lambda$. Let us start with writing down an equation for $h^{\lambda}$. Denote by $A$ the generator of the Ornstein-Uhlenbeck process (3.1),

$$
A=\Delta^{\mathcal{T}}-\frac{u}{2} \cdot \nabla, \quad \text { where } \quad \Delta^{\mathcal{T}}:=2 \sum_{m \in \mathcal{C}} \mathcal{T}_{m} \partial_{u_{m} \bar{u}_{m}}^{2} \cdot{ }^{18}
$$

Then, the generator of the effective equation (2.37) takes the form $A+\lambda B$, where

$$
B:=g \cdot \nabla \quad \text { and } \quad g=\left(g_{m}\right)_{m \in \mathcal{C}}, \quad g_{m}:=i \nabla_{m} H^{\mathrm{res}}=i \sum_{n:|n-m|=1} \nabla_{m} V_{m n}^{\mathrm{res}} .
$$

The density $\rho^{\lambda}$ satisfies in the weak sense the inverse Kolmogorov equation

$$
\left(A^{*}+\lambda B^{*}\right) \rho^{\lambda}=0,
$$

where

$$
A^{*}:=\Delta^{\mathcal{T}}+\frac{u}{2} \cdot \nabla+|\mathcal{C}| \quad \text { and } \quad B^{*}=-g \cdot \nabla .
$$

To calculate the operator $B^{*}$, we have used the equality $\nabla \cdot g=0$, following from the identity $\nabla_{m} \cdot\left(i \nabla_{m} \psi\right) \equiv 0$, which holds for any function $\psi \in C^{2}\left(\mathbb{C}^{|\mathcal{C}|}, \mathbb{R}\right)$. Applying the operator $A^{*}$ to the both sides of (3.21), by the direct computation we find $A^{*} \rho^{\lambda}=\mathrm{e}^{-|u|_{\mathcal{T}}^{2}} A h^{\lambda}$. Then, substituting (3.21) in (3.23) and multiplying the resulting equation by $\mathrm{e}^{|u|_{\mathcal{T}}^{2}}$, we get

$$
\left(A+\lambda \widetilde{B}^{*}\right) h^{\lambda}=0, \quad \text { where } \widetilde{B}^{*} h^{\lambda}:=\mathrm{e}^{|u|_{\mathcal{T}}^{2}} B^{*}\left(\mathrm{e}^{-|u|_{\mathcal{T}}^{2}} h^{\lambda}\right) .
$$

Now we will decompose Eq. (3.24) in the small parameter $\lambda$, then find a solution of the obtained system till order $\lambda$ and show that the rest is small. Let us start with a formal computation. Put

$$
h^{\lambda}=1+\lambda f+\lambda \widetilde{f}^{\lambda},
$$

where $f$ does not depend on $\lambda$ and $\tilde{f}^{\lambda}=o(1)$ as $\lambda \rightarrow 0$. Substituting (3.25) in (3.24), in order $\lambda^{0}$ we have the identity $A 1=0$, while in the order $\lambda$ we obtain

$$
A f=-\widetilde{B}^{*} 1 \text {. }
$$

\footnotetext{
18 In the real $(p, q)$-coordinates the operator $\Delta^{\mathcal{T}}$ has the form of the weighted laplacian $\Delta^{\mathcal{T}}=\frac{1}{2} \sum_{m \in \mathcal{C}} \mathcal{T}_{m}\left(\partial_{p_{m}^{2}}^{2}+\partial_{q_{m}^{2}}^{2}\right)$.
} 
To find the function $\widetilde{f}_{\lambda}$, we solve Eq. (3.26) and put

$$
\tilde{f}^{\lambda}:=\lambda^{-1}\left(h^{\lambda}-1-\lambda f\right)
$$

so that (3.25) becomes an identity.

Proposition 3.9. (i) Equation (3.26) has a solution $f$ of the form claimed in the formulation of the lemma.

(ii) The function $\tilde{f}^{\lambda}$, defined in (3.27) with $f$ from item (i), satisfies

$$
\left\langle\mu^{0}, \tilde{f}^{\lambda} \xi\right\rangle \rightarrow 0 \quad \text { as } \quad \lambda \rightarrow 0 \text { uniformly in } \mathcal{C},
$$

where $\xi$ is a function satisfying the conditions of the lemma.

Take $f$ and $\tilde{f}^{\lambda}$ as in Proposition 3.9. Then, using that $\left\langle\mu^{0}, \xi\right\rangle=0$, and (3.25), we obtain

$$
\left\langle\mu^{\lambda}, \xi\right\rangle=\left\langle\mu^{0}, h^{\lambda} \xi\right\rangle=\lambda\left\langle\mu^{0}, f \xi\right\rangle+o(\lambda),
$$

where $o(\lambda)=\lambda\left\langle\mu^{0}, \widetilde{f}^{\lambda} \xi\right\rangle$, so that we get the desired decomposition (3.13).

Proof of Proposition 3.9. Item (i). Using that for any function $\psi(u) \in C^{1}$ $\left(\mathbb{C}^{|\mathcal{C}|}, \mathbb{R}\right)$ we have $\left(i \nabla_{m} \psi\right) \cdot u_{m}=-\partial_{\varphi_{m}} \psi$, and the symmetry (3.7), we calculate

$$
\begin{aligned}
\widetilde{B}^{*} 1 & =2 \sum_{m \in \mathcal{C}} \mathcal{T}_{m}^{-1} g_{m} \cdot u_{m}=2 \sum_{m \in \mathcal{C}} \mathcal{T}_{m}^{-1} \sum_{n:|n-m|=1} i \nabla_{m} V_{m n}^{\mathrm{res}} \cdot u_{m} \\
& =-2 \sum_{|n-m|=1} \mathcal{T}_{m}^{-1} \partial_{\varphi_{m}} V_{m n}^{\mathrm{res}}=\sum_{|n-m|=1}\left(\mathcal{T}_{n}^{-1}-\mathcal{T}_{m}^{-1}\right) \partial_{\varphi_{m}} V_{m n}^{\mathrm{res}} \\
& =\frac{1}{2} \sum_{|n-m|=1}\left(\mathcal{T}_{n}^{-1}-\mathcal{T}_{m}^{-1}\right) \mathcal{J}_{m n}^{\mathrm{res}}
\end{aligned}
$$

where in the last equality we have employed Proposition 3.3.i. Take the solution $f_{m n}$ of

$$
A^{m n} f_{m n}=\left(\mathcal{T}_{m}^{-1}-\mathcal{T}_{n}^{-1}\right) \mathcal{J}_{m n}^{\mathrm{res}}=\frac{\mathcal{T}_{n}-\mathcal{T}_{m}}{\mathcal{T}_{n} \mathcal{T}_{m}} \mathcal{J}_{m n}^{\mathrm{res}},
$$

obtained in Lemma 3.2. Put

$$
f:=\frac{1}{2} \sum_{|m-n|=1} f_{m n} .
$$

Since Supp $f_{m n} \subseteq\{m, n\}$, we obviously have $A f_{m n}=A^{m n} f_{m n}$. Then, (3.31) joined with (3.30) implies (3.26). Since $\mathcal{J}_{m n}^{\text {res }}=-\mathcal{J}_{n m}^{\text {res }}$ and $A^{m n}=A^{n m}$, equations for $f_{m n}$ and $f_{n m}$ coincide, so that we get $f_{m n}=f_{n m}$.

Item (ii). We will need the following two identities. Take any function $\eta \in W_{2, l o c}^{2}\left(\mathbb{C}^{|\mathcal{C}|}, \mathbb{R}\right)$ such that $\eta$ and $\nabla \eta$ have at most a polynomial growth.

1. Since $\mu^{\lambda}$ is a stationary measure of the effective equation (2.37) and $A+\lambda B$ is the generator of the latter, we have $(A+\lambda B)^{*} \mu^{\lambda}=0$. So that, $\left\langle\mu^{\lambda}, A \eta\right\rangle=-\lambda\left\langle\mu^{\lambda}, B \eta\right\rangle$.

2. The integration by parts implies $\left\langle\mu^{0}, \widetilde{B}^{*} 1 \eta\right\rangle=\left\langle\mu^{0}, B \eta\right\rangle$. 
Also let us note that Proposition 3.5 still holds if replace the operator $A^{k j}$ by $A$ and the sum over $l \in\{k, j\}$ by the sum over $l \in \mathcal{C}$.

Now let us take the solution $\eta$ of equation $A^{k j} \eta=\xi$, obtained in Lemma 3.2. Since Supp $\eta \subseteq\{k, j\}$, we have $A \eta=A^{k j} \eta=\xi$. Using (3.27), (3.20), the version of Proposition 3.5 above, (3.26) and the identities (1), (2) above, we get

$$
\begin{aligned}
\left\langle\mu^{0}, \tilde{f}^{\lambda} \xi\right\rangle & =\left\langle\mu^{0}, \widetilde{f}^{\lambda} A \eta\right\rangle=\lambda^{-1}\left(\left\langle\mu^{0}, h^{\lambda} A \eta\right\rangle-\left\langle\mu^{0},(1+\lambda f) A \eta\right\rangle\right) \\
& =\lambda^{-1}\left(\left\langle\mu^{\lambda}, A \eta\right\rangle-\left\langle\mu^{0}, A(1+\lambda f) \eta\right\rangle\right) \\
& =-\left\langle\mu^{\lambda}, B \eta\right\rangle+\left\langle\mu^{0}, \widetilde{B}^{*} 1 \eta\right\rangle=-\left\langle\mu^{\lambda}-\mu^{0}, B \eta\right\rangle .
\end{aligned}
$$

Since the function $\xi$ is $C^{1}$-smooth, Lemma 3.2.ii implies that the solution $\eta$ also is. So that, the function $B \eta$ is continuous. Then, Proposition 3.1.ii implies

$$
\left\langle\mu^{\lambda}-\mu^{0}, B \eta\right\rangle \rightarrow 0 \quad \text { as } \lambda \rightarrow 0 .
$$

Recall that the uniformity in $\mathcal{C}$ of convergence in Proposition 3.1.ii is understood, as usual, in the sense of finite-dimensional projections. Then, to prove that convergence (3.33) holds uniformly in $\mathcal{C}$, it suffices to find a bounded set $\Lambda \subset \mathbb{Z}^{d}$, independent from the choice of the set $\mathcal{C}$, such that $\operatorname{Supp} B \eta \subseteq \Lambda$. Since $\operatorname{Supp} \eta \subseteq\{k, j\}$, we have $B \eta=g_{j} \cdot \nabla_{j} \eta+g_{k} \cdot \nabla_{k} \eta$. Since for any $m$, we have $\operatorname{Supp} g_{m} \subseteq\{n:|m-n| \leq 1\}$, the set $\Lambda$ can be chosen as $\Lambda=\left\{n \in \mathbb{Z}^{d}:|n-j| \wedge|n-k| \leq 1\right\}$.

Proof of Proposition 3.8. In this proof, we accept the following agreement: saying that something is "uniform in $\mathcal{T}$ ", we mean the uniformity in the temperatures $\left(\mathcal{T}_{k}, \mathcal{T}_{j}\right)$, if they belong to a compact subset of $\mathbb{R}_{+}^{2}$.

Since the resonant energy flow $\mathcal{J}_{k j}^{\text {res }}$ is independent from the temperatures, $\widetilde{\mathcal{J}}_{k j}^{\text {res }}$ depends on them only through the function $u(v)$, so the desired properties of the function $\widetilde{\mathcal{J}}_{k j}^{\text {res }}$ follow. Let us turn to the function $\widetilde{\eta}_{k j}$. Since $\eta_{k j}$ is a solution of Eq. (3.8) obtained in Lemma 3.2, due to formula (3.5), we have

$$
\eta_{k j}(u)=-\int_{0}^{\infty} \mathfrak{P}_{\tau}^{0} \mathcal{J}_{k j}^{\mathrm{res}}(u) \mathrm{d} \tau .
$$

Since the change of variables $u \mapsto v$ transforms the Ornstein-Uhlenbeck process (3.1) to

$$
\dot{v}_{j}=-\frac{v_{j}}{2}+\dot{\boldsymbol{\beta}}_{j}, \quad j \in \mathcal{C},
$$

relation (3.34) implies

$$
\widetilde{\eta}_{k j}(v)=-\int_{0}^{\infty} \widetilde{\mathfrak{P}}_{\tau}^{0} \widetilde{\mathcal{J}}_{k j}^{\mathrm{res}}(v) \mathrm{d} \tau,
$$

where $\widetilde{\mathfrak{P}}_{\tau}^{0}$ is a Markov semigroup associated with the process (3.35). Note that the operator $\widetilde{\mathfrak{P}}_{\tau}^{0}$ does not depend on the temperatures. Put $\partial_{\mathcal{T}}:=\partial_{\mathcal{T}_{k}^{l_{1}}}^{l_{1}+l_{2}} \mathcal{T}_{j}^{l_{2}}$, 
where $0 \leq l_{1}+l_{2} \leq r-1$. To prove that the function $\widetilde{\eta}_{k j}$ is $(r-1)$-time differentiable in $\mathcal{T}_{k}, \mathcal{T}_{j}$, we will just show that

$$
\partial_{\mathcal{T}} \widetilde{\eta}_{k j}(v)=-\int_{0}^{\infty} \widetilde{\mathfrak{P}}_{\tau}^{0} \partial_{\mathcal{T}} \widetilde{\mathcal{J}}_{k j}^{\mathrm{res}}(v) \mathrm{d} \tau,
$$

where the last integral converges. Changing the variables $u \mapsto v$ in the identity of Proposition 3.3.ii, we get $\left\langle\widetilde{\mu}^{0}, \widetilde{\mathcal{J}}_{k j}^{\text {res }}\right\rangle=0$. This holds for any $\mathcal{T}_{k}, \mathcal{T}_{j}>0$, so that we have $\left\langle\widetilde{\mu}^{0}, \partial_{\mathcal{T}} \widetilde{\mathcal{J}}_{k j}^{\text {res }}\right\rangle=0$. Since $\mu^{0}$ is a unique stationary measure of the Ornstein-Uhlenbeck process (3.1), the measure $\widetilde{\mu}^{0}$ is that for Eq. (3.35). The mixing property of the latter implies

$$
\left|\widetilde{\mathfrak{P}}_{\tau}^{0} \partial_{\mathcal{T}} \widetilde{\mathcal{J}}_{k j}^{\mathrm{res}}(v)\right|=\left|\widetilde{\mathfrak{P}}_{\tau}^{0} \partial_{\mathcal{T}} \widetilde{\mathcal{J}}_{k j}^{\mathrm{res}}(v)-\left\langle\widetilde{\mu}^{0}, \partial_{\mathcal{T}} \widetilde{\mathcal{J}}_{k j}^{\mathrm{res}}\right\rangle\right| \leq C\left(1+|v|^{m}\right) \mathrm{e}^{-b \tau},
$$

for any $\tau \geq 0$ and some constants $C, m, b>0$. It is possible to show that the constants $C, m$ and $b$ may be chosen uniformly in $\mathcal{T}$. It follows that the integral from the r.h.s. of (3.37) converges uniformly in $\mathcal{T}$. Then, it is not difficult to show that $\int_{0}^{\infty} \widetilde{\mathfrak{P}}_{\tau}^{0} \partial_{\mathcal{T}} \widetilde{\mathcal{J}}_{k j}^{\text {res }} \mathrm{d} \tau=\partial_{\mathcal{T}} \int_{0}^{\infty} \widetilde{\mathfrak{P}}_{\tau}^{0} \widetilde{\mathcal{J}}_{k j}^{\text {res }} \mathrm{d} \tau$. So that, applying $\partial_{\mathcal{T}}$ to the both sides of (3.36), we get (3.37).

Relation (3.37) joined with (3.38) implies that the function $\partial_{\mathcal{T}} \widetilde{\eta}_{k j}(v)$ has at most a polynomial growth in $v$, which is uniform in $\mathcal{T}$. The proof of the fact that the function $\partial_{\mathcal{T}} \widetilde{\eta}_{k j}$ is continuous with respect to $\left(v, \mathcal{T}_{k}, \mathcal{T}_{j}\right)$ is not complicated. Using that solutions of Eq. (3.35) depend on initial conditions in a continuous way, one should show that the r.h.s. of (3.37) with the time integral $\int_{0}^{\infty} \ldots \mathrm{d} \tau$ replaced by $\int_{0}^{T} \ldots \mathrm{d} \tau$ is continuous in $\left(v, \mathcal{T}_{k}, \mathcal{T}_{j}\right)$, and then observe that the rest $\int_{T}^{\infty} \ldots \mathrm{d} \tau$ is small, in view of (3.38).

\subsection{Examples}

1. Consider the quadratic interaction potential

$$
V\left(q_{j}, q_{k}\right)=\left(q_{j}-q_{k}\right)^{2} .
$$

It turns out that in this case the conductivity $\kappa\left(\mathcal{T}_{j}, \mathcal{T}_{k}\right)$ from Theorem 3.4 does not depend on the temperatures, so is a positive constant. We skip the proof, since next we will consider a similar but more complicated situation.

2. Let us now calculate the conductivity for the interaction potential

$$
V\left(q_{j}, q_{k}\right)=\left(q_{j}-q_{k}\right)^{4} .
$$

The potential $V$ has growth of power four, so assumption $H V$ is violated. Despite this, we have the following result.

Proposition 3.10. (i) Equation (2.25) is exponentially mixing with uniform in $\varepsilon$ rate (in the sense as in Theorem A.1).

(ii) There exists $\varepsilon_{0}>0$ such that for any $0<\varepsilon<\varepsilon_{0}, 0<\lambda \leq 1, \tau \geq 0$ and $m \geq 0$ we have

$\mathbf{E} \max _{s \in[\tau, \tau+1]}\left(|p(s)|^{2}+|q(s)|^{2}\right)^{m} \leq C(|\mathcal{C}|, m), \quad\left\langle\mu,\left(|p|^{2}+|q|^{2}\right)^{m}\right\rangle \leq C(|\mathcal{C}|, m)$, 
where $(p, q)(\tau)$ is a solution of Eq. (2.25) satisfying $\mathcal{D}(p, q)(0)=\mathcal{D}\left(p_{0}, q_{0}\right)$, and $\mu$ is its unique stationary measure.

Proof. (i) By the same arguments that was used in the proof of Theorem A.1.

(ii) By applying the Ito formula to the function $\left(H^{\nu}+\frac{\varepsilon}{2} \sum_{j \in \mathcal{C}} p_{j} q_{j}\right)^{m} \cdot{ }^{19}$

As it was explained in Remark 1.9, Proposition 3.10 implies that all theorems proved in this and previous chapters remain valid, except the uniformity of convergences in $\mathcal{C}$ which fails.

Proposition 3.11. The conductivity $\kappa$ has the form

$$
\kappa\left(\mathcal{T}_{j}, \mathcal{T}_{k}\right)=C\left(\mathcal{T}_{j}+\mathcal{T}_{k}\right)^{2},
$$

where the constant $C$ is independent from the temperatures.

Proof. By the direct computation, we get

$$
V_{k j}^{\mathrm{res}}(u)=\frac{3}{8}\left|u_{j}-u_{k}\right|^{4} .
$$

To calculate the conductivity $\kappa$, defined by formula (3.10), we need to find the solution $\eta_{k j}$ of Eq. (3.8), satisfying properties (a)-(c) from Lemma 3.2. Writing the operator $A^{k j}$ in the polar coordinates, we see that it commutes with the operator $\partial_{\varphi_{l}}$, for any $l \in \mathcal{C}$. Then, in view of the relation $\mathcal{J}_{k j}^{\text {res }}=2 \partial_{\varphi_{k}} V_{k j}^{\text {res }}$ established in Proposition 3.3.i, it suffices to find a solution $\phi_{k j}$ of equation

$$
A^{k j} \phi_{k j}=2 V_{k j}^{\mathrm{res}}+C\left(\mathcal{T}_{k}, \mathcal{T}_{j}\right),
$$

for some constant $C\left(\mathcal{T}_{k}, \mathcal{T}_{j}\right)$ (possibly depending on the temperatures $\mathcal{T}_{j}, \mathcal{T}_{k}$ ), such that the function $\eta_{k j}:=\partial_{\varphi_{k}} \phi_{k j}$ satisfies the conditions (a)-(c) from Lemma 3.2. By the direct computation, we get that the function

$$
\phi_{k j}=-\frac{3}{16}\left|u_{j}-u_{k}\right|^{4}-\frac{3}{2}\left(\mathcal{T}_{j}+\mathcal{T}_{k}\right)\left|u_{j}-u_{k}\right|^{2}
$$

satisfies (3.40). Using that $\partial_{\varphi_{k}}\left(\left|u_{j}-u_{k}\right|^{2}\right)=-2\left(i u_{k}\right) \cdot u_{j}$, we find

$$
\eta_{k j}=\partial_{\varphi_{k}} \phi_{k j}=\frac{3}{4}\left(i u_{k}\right) \cdot u_{j}\left(\left|u_{j}-u_{k}\right|^{2}+4\left(\mathcal{T}_{j}+\mathcal{T}_{k}\right)\right) .
$$

It is not difficult to see that the function $\eta_{k j}$ satisfies the required conditions. Note also that

$$
\mathcal{J}_{k j}^{\mathrm{res}}=2 \partial_{\varphi_{k}} V_{k j}^{\mathrm{res}}=-3\left(i u_{k}\right) \cdot u_{j}\left|u_{j}-u_{k}\right|^{2} .
$$

Substituting (3.41) and (3.42) to the first representation for the conductivity $\kappa$ from (3.10), passing to the coordinates $r_{j}:=\left|u_{j}\right|, r_{k}:=\left|u_{k}\right|, \psi=\varphi_{j}-\varphi_{k}$, and using that

$$
\left|u_{j}-u_{k}\right|^{2}=r_{k}^{2}+r_{j}^{2}-2 r_{k} r_{j} \cos \psi \quad \text { and }\left(i u_{k}\right) \cdot u_{j}=r_{k} r_{j} \sin \psi,
$$

we get the desired result by the direct computation.

${ }^{19}$ For the both items, one should use that

$$
\left\{\frac{1}{2} \sum_{j \in \mathcal{C}} p_{j} q_{j}, \frac{1}{2} \sum_{|k-j|=1}\left(q_{k}-q_{j}\right)^{4}\right\}=-\sum_{|k-j|=1}\left(q_{k}-q_{j}\right)^{4} .
$$




\subsection{Space-Time Correlations of the Energy Flow}

In this section, we study stationary space-time correlations of the energy flow under the limits $\varepsilon, \lambda \rightarrow 0$ and investigate their connection with the conductivity $\kappa$. Since till the end of the section, we will not talk about the uniformity of convergences with respect to the choice of the set $\mathcal{C}$, further on constants are permitted to depend on it.

Theorem 3.12. Let $\mathfrak{P}_{\tau}^{\varepsilon, \lambda}$ be a Markov semigroup associated with Eq. (2.25) and $\mu^{\varepsilon, \lambda}$ be its unique stationary measure. Take any $k, j, m, l \in \mathcal{C}$ satisfying $|k-j|=|m-l|=1$ and denote

$$
\mathcal{Y}^{\varepsilon, \lambda}:=\int_{0}^{\infty}\left\langle\mu^{\varepsilon, \lambda}, \mathcal{J}_{k j} \mathfrak{P}_{\tau}^{\varepsilon, \lambda} \mathcal{J}_{m l}\right\rangle-\left\langle\mu^{\varepsilon, \lambda}, \mathcal{J}_{k j}\right\rangle\left\langle\mu^{\varepsilon, \lambda}, \mathcal{J}_{m l}\right\rangle \mathrm{d} \tau
$$

Then

$$
\lim _{\lambda \rightarrow 0} \lim _{\varepsilon \rightarrow 0} \mathcal{Y}^{\varepsilon, \lambda}=\left\{\begin{array}{lll}
\mathcal{T}_{j} \mathcal{T}_{k} \kappa\left(\mathcal{T}_{j}, \mathcal{T}_{k}\right) & \text { if } & m=k, l=j, \\
-\mathcal{T}_{j} \mathcal{T}_{k} \kappa\left(\mathcal{T}_{j}, \mathcal{T}_{k}\right) & \text { if } & m=j, l=k, \\
0 & \text { if } & \{k, j\} \neq\{m, l\} .
\end{array}\right.
$$

Corollary 3.13. Assertion of Theorem 1.8 is satisfied.

Proof of Corollary 3.13. Let us first note that for the constant temperature profile $\mathcal{T}_{n}=\hat{\mathcal{T}} \forall n \in \mathcal{C}$ we have $\left\langle\mu^{\varepsilon, \lambda}, \mathcal{J}_{k j}\right\rangle=0$, for any $k, j \in \mathcal{C},|k-j|=1$. Indeed, since in the present case the measure $\mu^{\varepsilon, \lambda}$ is just the Gibbs measure, $\mu^{\varepsilon, \lambda}=Z^{-1}(\hat{\mathcal{T}}) \mathrm{e}^{-H^{\nu} / \hat{\mathcal{T}}} \mathrm{dLeb}$, this follows from evenness of the Hamiltonian $H^{\nu}$ with respect to the variable $p$, and oddness of the energy flow $\mathcal{J}_{k j}$ with respect to it.

Now let us rewrite the r.h.s. of (1.18) as

$$
\begin{aligned}
& \frac{1}{\hat{\mathcal{T}}^{2} N} \lim _{\lambda \rightarrow 0} \lim _{\varepsilon \rightarrow 0}\left(\sum_{j=0}^{N-1} \int_{0}^{\infty}\left\langle\mu^{\varepsilon, \lambda}, \mathcal{J}_{j j+1} \mathfrak{P}_{\tau}^{\varepsilon, \lambda} \mathcal{J}_{j j+1}\right\rangle \mathrm{d} \tau\right. \\
& \left.\quad+\sum_{0 \leq j, k \leq N-1, j \neq k} \int_{0}^{\infty}\left\langle\mu^{\varepsilon, \lambda}, \mathcal{J}_{j j+1} \mathfrak{P}_{\tau}^{\varepsilon, \lambda} \mathcal{J}_{k k+1}\right\rangle \mathrm{d} \tau\right) .
\end{aligned}
$$

Due to Theorem 3.12, the limits of the first summand in the brackets equal to $N \hat{\mathcal{T}}^{2} \hat{\kappa}(\hat{\mathcal{T}})$, while the limits of the second one vanish.

Proof of Theorem 3.12. The proof of the theorem is based on the following auxiliary lemma, which we will establish in the end of the section. Denote by $\mathfrak{P}_{\tau}^{\lambda}$ and $\mathfrak{P}_{\tau}^{0}$ the Markov semigroups associated with the effective equation (2.37) and the Ornstein-Uhlenbeck process (3.1) correspondingly. Let

$$
\begin{aligned}
\eta_{m l}^{\varepsilon, \lambda}(u) & :=\int_{0}^{\infty} \mathfrak{P}_{\tau}^{\varepsilon, \lambda} \mathcal{J}_{m l}(u)-\left\langle\mu^{\varepsilon, \lambda}, \mathcal{J}_{m l}\right\rangle \mathrm{d} \tau, \\
\eta_{m l}^{\lambda}(u) & :=\int_{0}^{\infty} \mathfrak{P}_{\tau}^{\lambda} \mathcal{J}_{m l}^{\mathrm{res}}(u)-\left\langle\mu^{\lambda}, \mathcal{J}_{m l}^{\mathrm{res}}\right\rangle \mathrm{d} \tau,
\end{aligned}
$$




$$
\eta_{m l}(u):=-\int_{0}^{\infty} \mathfrak{P}_{\tau}^{0} \mathcal{J}_{m l}^{\mathrm{res}}(u) \mathrm{d} \tau,{ }^{20}
$$

where $\mu^{\lambda}$ is the unique stationary measure of Eq. (2.37). Note that, due to (3.5), the function $\eta_{m l}$ coincides with that from Theorem 3.4. Put also

$$
\hat{\eta}_{m l}^{\varepsilon, \lambda}(u):=\int_{0}^{\infty}\left|\mathfrak{P}_{\tau}^{\varepsilon, \lambda} \mathcal{J}_{m l}(u)-\left\langle\mu^{\varepsilon, \lambda}, \mathcal{J}_{m l}\right\rangle\right| \mathrm{d} \tau,
$$

and define $\hat{\eta}_{m l}^{\lambda}(u)$ and $\hat{\eta}_{m l}(u)$ in a similar way.

Lemma 3.14. (i) There exist constants $C, n>0$, independent from $\varepsilon$ and $\lambda$, such that for any $u \in \mathbb{C}^{|\mathcal{C}|}$ we have

$$
\left|\eta_{m l}^{\varepsilon, \lambda}(u)\right|, \hat{\eta}_{m l}^{\varepsilon, \lambda}(u),\left|\eta_{m l}^{\lambda}(u)\right|, \hat{\eta}_{m l}^{\lambda}(u),\left|\eta_{m l}(u)\right|, \hat{\eta}_{m l}(u) \leq C\left(1+|u|^{n}\right) .
$$

(ii) For any $u \in \mathbb{C}^{|\mathcal{C}|}$, we have

$\eta_{m l}^{\varepsilon, \lambda}(u) \rightarrow \eta_{m l}^{\lambda}(u) \quad$ as $\varepsilon \rightarrow 0 \quad$ and $\quad \eta_{m l}^{\lambda}(u) \rightarrow-\eta_{m l}(u) \quad$ as $\quad \lambda \rightarrow 0$,

where the both convergences hold uniformly in $u \in K$, if $K \subset \mathbb{C}^{|\mathcal{C}|}$ is a compact set.

(iii) For any $\theta \in[0,2 \pi)$ we have $\eta_{m l}^{\lambda}\left(\mathrm{e}^{i \theta} u\right) \equiv \eta_{m l}^{\lambda}(u)$.

Let us first show that the integral $\mathcal{Y}^{\varepsilon, \lambda}$ converges. Due to Fubini's theorem, for this purpose it suffices to establish that

$$
\left\langle\mu^{\varepsilon, \lambda}, \int_{0}^{\infty}\left|\mathcal{J}_{k j}\left(\mathfrak{P}_{\tau}^{\varepsilon, \lambda} \mathcal{J}_{m l}-\left\langle\mu^{\varepsilon, \lambda}, \mathcal{J}_{m l}\right\rangle\right)\right| \mathrm{d} \tau\right\rangle<\infty .
$$

Note that the l.h.s. of (3.44) equals to $\left\langle\mu^{\varepsilon, \lambda},\left|\mathcal{J}_{k j}\right| \hat{\eta}_{m l}^{\varepsilon, \lambda}\right\rangle$. Since the energy flow $\mathcal{J}_{k j}$ and the function $\hat{\eta}_{m l}^{\varepsilon, \lambda}$ have at most a polynomial growth, inequality (3.44) follows from the estimate for the stationary measure $\mu^{\varepsilon, \lambda}$ provided by Lemma 2.1.ii. Now let us pass to the limit $\varepsilon \rightarrow 0$. Fubini's theorem implies

$$
\mathcal{Y}^{\varepsilon, \lambda}=\left\langle\mu^{\varepsilon, \lambda}, \mathcal{J}_{k j} \eta_{m l}^{\varepsilon, \lambda}\right\rangle \text {. }
$$

Theorem 2.8.i joined with Lemma 3.14.i,ii implies

$$
\left\langle\mu^{\varepsilon, \lambda}, \mathcal{J}_{k j} \eta_{m l}^{\varepsilon, \lambda}\right\rangle \rightarrow\left\langle\mu^{\lambda}, \mathcal{J}_{k j} \eta_{m l}^{\lambda}\right\rangle \quad \text { as } \varepsilon \rightarrow 0 .
$$

In view of Proposition 2.12 and Lemma 3.14.iii, we have

$$
\left\langle\mu^{\lambda}, \mathcal{J}_{k j} \eta_{m l}^{\lambda}\right\rangle=\left\langle\mu^{\lambda},\left\langle\mathcal{J}_{k j} \eta_{m l}^{\lambda}\right\rangle_{R}\right\rangle=\left\langle\mu^{\lambda},\left\langle\mathcal{J}_{k j}\right\rangle_{R} \eta_{m l}^{\lambda}\right\rangle=\left\langle\mu^{\lambda}, \mathcal{J}_{k j}^{\text {res }} \eta_{m l}^{\lambda}\right\rangle .
$$

Now we pass to the limit $\lambda \rightarrow 0$. Proposition 3.1.ii joined with Lemma 3.14.ii implies

$$
\left\langle\mu^{\lambda}, \mathcal{J}_{k j}^{\mathrm{res}} \eta_{m l}^{\lambda}\right\rangle \rightarrow-\left\langle\mu^{0}, \mathcal{J}_{k j}^{\mathrm{res}} \eta_{m l}\right\rangle \quad \text { as } \lambda \rightarrow 0
$$

${ }^{20}$ Recall that $\left\langle\mu^{0}, \mathcal{J}_{m l}^{\text {res }}\right\rangle=0$, due to Proposition 3.3.ii, where $\mu^{0}$ is the unique stationary measure of the Ornstein-Uhlenbeck process (3.1). 
Combining (3.45)-(3.48), we see that it remains to establish the identity

$$
-\left\langle\mu^{0}, \mathcal{J}_{k j}^{\mathrm{res}} \eta_{m l}\right\rangle=\left\{\begin{array}{cll}
\mathcal{T}_{j} \mathcal{T}_{k} \kappa\left(\mathcal{T}_{j}, \mathcal{T}_{k}\right) & \text { if } & m=k, l=j \\
-\mathcal{T}_{j} \mathcal{T}_{k} \kappa\left(\mathcal{T}_{j}, \mathcal{T}_{k}\right) & \text { if } & m=j, l=k \\
0 & \text { if } & \{m, l\} \neq\{k, j\}
\end{array}\right.
$$

The first equality of (3.49) immediately follows from the formula (3.10) while the second one from the relation $\eta_{j k}=-\eta_{k j}$. The third equality follows from the rotation invariance of the Gaussian measure $\mu^{0}$ and can be obtained by the argument used in the proof of Proposition 3.7.

Proof of Lemma 3.14. Let us start with several auxiliary propositions.

Proposition 3.15. The effective equation (2.37) is exponentially mixing with the rate which is uniform in $0 \leq \lambda \leq 1$ (in the sense as in Theorem A.1).

Proof. It can be shown by an argument similar to that used in the proof of Theorem A.1, but simplified. Note only that in the proof of the recurrence one should apply the Ito formula to the function $\mathrm{e}^{\delta \tau} \sum_{j \in \mathcal{C}} I_{j}{ }^{21}$

Proposition 3.16. For any compact set $K \subset \mathbb{C}^{|\mathcal{C}|}$, the time integral from the definition of the function $\eta_{m l}^{\varepsilon, \lambda}$ converges uniformly in $u \in K$ and $\varepsilon$, while that from the definition of the function $\eta_{m l}^{\lambda}$ converges uniformly in $u \in K$ and $\lambda$.

Proof. Recall that in Appendix A, it is proven that Eq. (2.25) is exponentially mixing with the rate which is uniform in $\varepsilon$. Then, it can be shown that for all $u \in \mathbb{C}^{|\mathcal{C}|}$ we have

$$
\left|\mathfrak{P}_{\tau}^{\varepsilon, \lambda} \mathcal{J}_{m l}(u)-\left\langle\mu^{\varepsilon, \lambda}, \mathcal{J}_{m l}\right\rangle\right| \leq C\left(1+|u|^{n}\right) \mathrm{e}^{-b \tau},
$$

where the constants $C, n, b>0$ are independent from $\varepsilon$ (see e.g. a proof of Theorem 1 from [37]). It follows that the integral $\int_{0}^{\infty} \mathfrak{P}_{\tau}^{\varepsilon, \lambda} \mathcal{J}_{m l}(u)-\left\langle\mu^{\varepsilon, \lambda}, \mathcal{J}_{m l}\right\rangle \mathrm{d} \tau$ converges uniformly in $\varepsilon$ and $u \in K$. To prove that the integral $\int_{0}^{\infty} \mathfrak{P}_{\tau}^{\lambda} \mathcal{J}_{m l}^{\text {res }}(u)-\left\langle\mu^{\lambda}, \mathcal{J}_{m l}^{\text {res }}\right\rangle \mathrm{d} \tau$ converges uniformly in $\lambda$ and $u \in K$, we use Proposition 3.15 and argue similarly.

Now we turn to the proof of the lemma.

Item (i). The estimates $\left|\eta_{m l}^{\varepsilon, \lambda}\right|, \hat{\eta}_{m l}^{\varepsilon, \lambda} \leq C\left(1+|u|^{n}\right)$ follow from (3.50). The other estimates can be obtained similarly.

Item (ii). Let us first show that $\eta_{m l}^{\varepsilon, \lambda}(u) \rightarrow \eta_{m l}^{\lambda}(u)$ as $\varepsilon \rightarrow 0$ uniformly in $u \in K$. Due to Proposition 3.16, it suffices to prove that for any $T>0$

$$
\int_{0}^{T} \mathfrak{P}_{\tau}^{\varepsilon, \lambda} \mathcal{J}_{m l}(u)-\left\langle\mu^{\varepsilon, \lambda}, \mathcal{J}_{m l}\right\rangle \mathrm{d} \tau \rightarrow \int_{0}^{T} \mathfrak{P}_{\tau}^{\lambda} \mathcal{J}_{m l}^{\text {res }}(u)-\left\langle\mu^{\lambda}, \mathcal{J}_{m l}^{\text {res }}\right\rangle \mathrm{d} \tau \quad \text { as } \quad \varepsilon \rightarrow 0
$$

uniformly in $u \in K$. Since, due to Theorem 2.8.i joined with Proposition 2.12, we have $\left\langle\mu^{\varepsilon, \lambda}, \mathcal{J}_{m l}\right\rangle \rightarrow\left\langle\mu^{\lambda}, \mathcal{J}_{m l}^{\text {res }}\right\rangle$ as $\varepsilon \rightarrow 0$, it suffices to establish that

21 This is related to the fact that the sum of actions $\sum_{j \in \mathcal{C}} I_{j}$ is a first integral of the resonant Hamiltonian $H^{\text {res }}$. The latter follows from the relation $\left\{\sum_{j \in \mathcal{C}} I_{j}, H^{\text {res }}\right\}=$ $-\sum_{j \in \mathcal{C}} \partial_{\varphi_{j}} H^{\mathrm{res}}=-\sum_{j \in \mathcal{C}} \sum_{k:|j-k|=1} \partial_{\varphi_{j}} V_{j k}^{\mathrm{res}}=-\sum_{|j-k|=1} \partial_{\varphi_{j}} V_{j k}^{\mathrm{res}}$, and Proposition C.1.iv. 


$$
\int_{0}^{T} \mathfrak{P}_{\tau}^{\varepsilon, \lambda} \mathcal{J}_{m l}(u) \mathrm{d} \tau \rightarrow \int_{0}^{T} \mathfrak{P}_{\tau}^{\lambda} \mathcal{J}_{m l}^{\text {res }}(u) \mathrm{d} \tau \quad \text { as } \quad \varepsilon \rightarrow 0
$$

uniformly in $u \in K$. Let us rewrite the l.h.s. of (3.51) as $\int_{0}^{T} \mathbf{E} \mathcal{J}_{m l}\left(u^{\varepsilon, \lambda}(\tau)\right) \mathrm{d} \tau$, where $u^{\varepsilon, \lambda}(\tau)$ is a solution of Eq. (2.25) satisfying $u^{\varepsilon, \lambda}(0)=u$. Denote by $a^{\varepsilon, \lambda}$ the process $u^{\varepsilon, \lambda}$ written in the $a$-variables.

Remark 3.17. Simple analysis of proofs of Lemma 4.1 and Theorem 2.4 shows that convergences obtained there for the process $a^{\varepsilon, \lambda}$ hold uniformly in $u \in K$, if the set $\mathcal{C}$ is fixed.

Applying Lemma 4.1 to the function $h(u, \theta):=\mathcal{J}_{m l}\left(\mathrm{e}^{i \theta} u\right)$, in view of Remark 3.17 we obtain that the integral above is uniformly in $u \in K$ close to $\int_{0}^{T} \mathbf{E} \mathcal{J}_{m l}^{\text {res }}\left(a^{\varepsilon, \lambda}(\tau)\right) \mathrm{d} \tau$. Due to Theorem 2.4, the latter integral is uniformly in $u \in K$ close to

$$
\int_{0}^{T} \mathbf{E} \mathcal{J}_{m l}^{\mathrm{res}}\left(u^{\lambda}(\tau)\right) \mathrm{d} \tau=\int_{0}^{T} \mathfrak{P}_{\tau}^{\lambda} \mathcal{J}_{m l}^{\mathrm{res}}(u) \mathrm{d} \tau,
$$

where $u^{\lambda}(\tau)$ is a solution of the effective equation (2.37) satisfying $u^{\lambda}(0)=u$.

The convergence $\eta_{m l}^{\lambda}(u) \rightarrow-\eta_{m l}(u)$ as $\lambda \rightarrow 0$ can be established with help of Proposition 3.1 in a similar way.

Item (iii). Let $u^{\lambda}(\tau)$ be a solution of the effective equation (2.37) satisfying $u^{\lambda}(0)=u$. The rotation invariance of the effective equation (see Proposition 2.11) implies that $\mathrm{e}^{i \theta} u^{\lambda}(\tau)$ is its weak solution. Since it satisfies $\mathrm{e}^{i \theta} u^{\lambda}(0)=$ $\mathrm{e}^{i \theta} u$, we have

$$
\begin{aligned}
\eta_{m l}^{\lambda}\left(\mathrm{e}^{i \theta} u\right) & =\int_{0}^{\infty} \mathbf{E} \mathcal{J}_{m l}^{\mathrm{res}}\left(\mathrm{e}^{i \theta} u^{\lambda}(\tau)\right)-\left\langle\mu^{\lambda}, \mathcal{J}_{m l}^{\mathrm{res}}\right\rangle \mathrm{d} \tau \\
& =\int_{0}^{\infty} \mathbf{E} \mathcal{J}_{m l}^{\mathrm{res}}\left(u^{\lambda}(\tau)\right)-\left\langle\mu^{\lambda}, \mathcal{J}_{m l}^{\mathrm{res}}\right\rangle \mathrm{d} \tau=\eta_{m l}^{\lambda}(u),
\end{aligned}
$$

where we have used Proposition C.1.i.

\section{Proofs of the Averaging Theorems}

Here, we prove Theorems 2.4, 2.8 and Proposition 4.9, which implies Proposition 2.7.

\subsection{Averaging Lemma}

We start with the following averaging lemma which is the main tools in the proofs of Theorems 2.4 and 2.8. Let $u^{\varepsilon}(\tau)$ be a solution of Eq. (2.25), either satisfying $\mathcal{D}\left(u^{\varepsilon}(0)\right)=\mathcal{D}\left(u_{0}\right)$ or a stationary one. Let $a^{\varepsilon}(\tau)$ be the corresponding $a$-variables, given by (2.27). Fix a bounded set $\Lambda \subset \mathbb{Z}^{d}$ and assume that the set $\mathcal{C}$ satisfies $\mathcal{C} \supseteq \Lambda$. Take a function $h(u, \theta) \in \mathcal{L}_{\text {loc }}\left(\mathbb{C}^{|\mathcal{C}|} \times[0,2 \pi)\right)$ such that for 
each $\theta$ the function $h(\cdot, \theta)$ satisfies $\operatorname{Supp} h(\cdot, \theta) \subseteq \Lambda$ and has at most a polynomial growth at infinity which is uniform in $\theta$. Then, there exist non-decreasing functions $c, c_{1}: \mathbb{R} \mapsto \mathbb{R}$ such that

$$
\begin{aligned}
\left|h\left(u_{1}, \theta\right)\right| & \leq c\left(\max _{j \in \Lambda}\left|u_{1 j}\right|\right), \\
\left|h\left(u_{1}, \theta\right)-h\left(u_{2}, \theta\right)\right| & \leq c_{1}\left(\max _{k=1,2, j \in \Lambda}\left|u_{k j}\right|\right) \sum_{j \in \Lambda}\left|u_{1 j}-u_{2 j}\right|,
\end{aligned}
$$

for any $u_{1}, u_{2} \in \mathbb{C}^{|\mathcal{C}|}, \theta \in[0,2 \pi)$, where the function $c$ has at most a polynomial growth at infinity. Denote

$$
\langle h\rangle_{\theta}(u):=\int_{0}^{2 \pi} h(u, \theta) \mathrm{d} \theta .
$$

Lemma 4.1. Take a function $h$ as above. Then

$$
\mathbf{E} \max _{0 \leq \tau \leq T}\left|\int_{0}^{\tau}\left(h\left(a^{\varepsilon}(s), \varepsilon^{-1} s\right)-\langle h\rangle_{\theta}\left(a^{\varepsilon}(s)\right)\right) \mathrm{d} s\right| \rightarrow 0 \text { as } \varepsilon \rightarrow 0,
$$

uniformly in $\mathcal{C}$ and with respect to the choice of the function hatisfying (4.1) for fixed $c, c_{1}$.

Proof. For purposes of the proof, we first introduce some notations. For an event $\Gamma$ and a random variable $\xi$, we denote

$$
\mathbf{E}_{\Gamma} \xi:=\mathbf{E}\left(\xi \mathbb{I}_{\bar{\Gamma}}\right) .
$$

By $æ(r)$, we denote various functions of $r \in \mathbb{R}$ such that $æ(r) \rightarrow 0$ as $r \rightarrow \infty$ uniformly in $\mathcal{C}$ and $h$, satisfying (4.1) for fixed $c, c_{1}$. By $æ_{\infty}(r)$, we denote functions $æ(r)$ such that $æ(r)=o\left(r^{-m}\right)$ uniformly in $\mathcal{C}$ and $h$, for each $m>0$. We write $æ(r)=æ(r ; a)$ to indicate that $æ(r)$ depends on a parameter $a$.

Denote by $U_{1}(\Lambda)$ the neighbourhood of radius 1 of $\Lambda$ in $\mathcal{C}$, i.e.

$$
U_{1}(\Lambda):=\{n \in \mathcal{C} \mid \text { there exists } k \in \Lambda \text { satisfying }|n-k| \leq 1\} .
$$

Fix $R>0$. Set

$$
\Omega_{R}=\left\{\max _{k \in U_{1}(\Lambda)} \max _{0 \leq \tau \leq T}\left|a_{k}^{\varepsilon}(\tau)\right| \geq R\right\} .
$$

Due to the estimate (i) of Lemma 2.1, we have

$$
\mathbf{P}\left(\Omega_{R}\right) \leq æ_{\infty}(R) .
$$

In view of the polynomial growth of the function $h$,

$$
\mathbf{E}_{\bar{\Omega}_{R}} \max _{0 \leq \tau \leq T}\left|\int_{0}^{\tau} h\left(a^{\varepsilon}(s), \varepsilon^{-1} s\right) \mathrm{d} s\right| \leq æ_{\infty}(R),
$$

and the function $\langle h\rangle_{\theta}\left(a^{\varepsilon}(s)\right)$ satisfies a similar relation. Then, it is sufficient to show that for any $R \geq 0$ 


$$
\mathcal{U}^{\varepsilon}:=\mathbf{E}_{\Omega_{R}} \max _{0 \leq \tau \leq T}\left|\int_{0}^{\tau} h\left(a^{\varepsilon}(s), \varepsilon^{-1} s\right)-\langle h\rangle_{\theta}\left(a^{\varepsilon}(s)\right) \mathrm{d} s\right| \rightarrow 0 \quad \text { as } \quad \varepsilon \rightarrow 0
$$

uniformly in $\mathcal{C}, h$. For this purpose, we consider a partition of the interval $[0, T]$ to subintervals of the length $\delta=\sqrt{\varepsilon}$ by the points $\tau_{l}=l \delta, 0 \leq l \leq L$, where $L=[T / \delta]$. Denote

$$
\eta_{l}=\int_{\tau_{l}}^{\tau_{l+1}} h\left(a^{\varepsilon}(s), \varepsilon^{-1} s\right)-\langle h\rangle_{\theta}\left(a^{\varepsilon}(s)\right) \mathrm{d} s .
$$

Then

$$
\mathcal{U}^{\varepsilon} \leq \mathbf{E}_{\Omega_{R}} \sum_{l=0}^{L-1}\left|\eta_{l}\right|+2 \delta c(R)
$$

We have

$$
\begin{aligned}
\left|\eta_{l}\right| \leq & \left|\int_{\tau_{l}}^{\tau_{l+1}} h\left(a^{\varepsilon}(s), \varepsilon^{-1} s\right)-h\left(a^{\varepsilon}\left(\tau_{l}\right), \varepsilon^{-1} s\right) \mathrm{d} s\right| \\
& +\left|\int_{\tau_{l}}^{\tau_{l+1}} h\left(a^{\varepsilon}\left(\tau_{l}\right), \varepsilon^{-1} s\right)-\langle h\rangle_{\theta}\left(a^{\varepsilon}\left(\tau_{l}\right)\right) \mathrm{d} s\right| \\
& +\left|\int_{\tau_{l}}^{\tau_{l+1}}\langle h\rangle_{\theta}\left(a^{\varepsilon}\left(\tau_{l}\right)\right)-\langle h\rangle_{\theta}\left(a^{\varepsilon}(s)\right) \mathrm{d} s\right|=: \mathcal{Y}_{l}^{1}+\mathcal{Y}_{l}^{2}+\mathcal{Y}_{l}^{3} .
\end{aligned}
$$

Terms $\mathcal{Y}_{l}^{1}, \mathcal{Y}_{l}^{3}$. Since the function $h$ is locally Lipschitz,

$$
\mathbf{E}_{\Omega_{R}}\left(\mathcal{Y}_{l}^{1}+\mathcal{Y}_{l}^{3}\right) \leq 2 \delta c_{1}(R) \sum_{j \in \Lambda} \mathbf{E}_{\Omega_{R}} \max _{\tau_{l} \leq s \leq \tau_{l+1}}\left|a_{j}^{\varepsilon}(s)-a_{j}^{\varepsilon}\left(\tau_{l}\right)\right| \leq \delta æ\left(\delta^{-1} ; R\right),
$$

where the last inequality follows from Eq. (2.29).

Term $\mathcal{Y}_{l}^{2}$. Changing the variable of integration $\hat{s}:=\varepsilon^{-1} s$, we obtain

$$
\mathbf{E}_{\Omega_{R}} \mathcal{Y}_{2}=\varepsilon \mathbf{E}_{\Omega_{R}}\left|\int_{\varepsilon^{-1} \tau_{l}}^{\varepsilon^{-1} \tau_{l+1}} h\left(a^{\varepsilon}\left(\tau_{l}\right), \hat{s}\right)-\langle h\rangle_{\theta}\left(a^{\varepsilon}\left(\tau_{l}\right)\right) \mathrm{d} \hat{s}\right| \leq 2 \varepsilon c(R),
$$

due to the definition of the function $\langle h\rangle_{\theta}$.

In view of the identity $\varepsilon=\delta^{2}$, estimates (4.5) and (4.6) imply

$$
\mathbf{E}_{\Omega_{R}}\left|\eta_{l}\right| \leq \delta\left(æ\left(\delta^{-1} ; R\right)+2 \delta c(R)\right) .
$$

Noting that $L \leq T \delta^{-1}$, from (4.4) we get $\mathcal{U}^{\varepsilon} \leq T\left(æ\left(\delta^{-1} ; R\right)+2 \delta c(R)\right)+2 \delta c(R) \rightarrow 0 \quad$ as $\quad \varepsilon \rightarrow 0 \quad$ uniformly in $\mathcal{C}, h$.

Let now $u^{\varepsilon}(\tau)$ be a stationary solution of Eq. (2.25). 
Corollary 4.2. Let a function $h \in \mathcal{L}_{\text {loc }}\left(\mathbb{C}^{|\mathcal{C}|}\right)$ satisfies $\operatorname{Supp} h \subseteq \Lambda$ and has at most a polynomial growth at infinity. Then, for any $\tau \geq 0$, we have

$$
\mathbf{E} h\left(u^{\varepsilon}(\tau)\right)-\mathbf{E} h\left(a^{\varepsilon}(\tau)\right) \rightarrow 0 \quad \text { as } \quad \varepsilon \rightarrow 0 \quad \text { uniformly in } \mathcal{C} .
$$

Proof. There exist functions $c, c_{1}: \mathbb{R} \mapsto \mathbb{R}$, where $c$ has at most a polynomial growth at infinity, such that

$$
\left|h\left(u_{1}\right)\right| \leq c\left(\max _{j \in \Lambda}\left|u_{1 j}\right|\right),\left|h\left(u_{1}\right)-h\left(u_{2}\right)\right| \leq c_{1}\left(\max _{k=1,2, j \in \Lambda}\left|u_{k j}\right|\right) \sum_{j \in \Lambda}\left|u_{1 j}-u_{2 j}\right|,
$$

for any $u_{1}, u_{2} \in \mathbb{C}^{|\mathcal{C}|}$. Consider the function $\hat{h}(u, \theta):=h\left(\mathrm{e}^{i \theta} u\right)$. Clearly, it satisfies (4.1) with $c, c_{1}$ from (4.7). Applying Lemma 4.1, we get

$$
\int_{0}^{T} \mathbf{E} h\left(u^{\varepsilon}(\tau)\right)-\mathbf{E}\langle h\rangle_{R}\left(a^{\varepsilon}(\tau)\right) \mathrm{d} \tau \rightarrow 0 \quad \text { as } \quad \varepsilon \rightarrow 0,
$$

uniformly in $\mathcal{C}$ and with respect to the choice of the function $h$ satisfying (4.7). In view of Proposition C.1.i, we have $\langle h\rangle_{R}\left(a^{\varepsilon}(\tau)\right) \equiv\langle h\rangle_{R}\left(u^{\varepsilon}(\tau)\right)$. Then, stationarity of the process $u^{\varepsilon}$ implies that the function under the integral in (4.8) is independent from time, so that for any $\tau \geq 0$ we have

$\mathbf{E} h\left(u^{\varepsilon}(\tau)\right)-\mathbf{E}\langle h\rangle_{R}\left(a^{\varepsilon}(\tau)\right) \rightarrow 0 \quad$ as $\quad \varepsilon \rightarrow 0 \quad$ uniformly in $\mathcal{C}$ and $h$.

Let $h^{\varepsilon, \tau}(u):=h\left(\mathrm{e}^{-i \varepsilon^{-1} \tau} u\right)$. Since the functions $h^{\varepsilon, \tau}$ satisfy (4.7) for any $\varepsilon$ and $\tau$, we have

$$
\mathbf{E} h^{\varepsilon, \tau}\left(u^{\varepsilon}(\tau)\right)-\mathbf{E}\left\langle h^{\varepsilon, \tau}\right\rangle_{R}\left(a^{\varepsilon}(\tau)\right) \rightarrow 0 \quad \text { as } \quad \varepsilon \rightarrow 0 \quad \text { uniformly in } \mathcal{C} \text {. }
$$

Since $h^{\varepsilon, \tau}\left(u^{\varepsilon}(\tau)\right) \equiv h\left(a^{\varepsilon}(\tau)\right)$ and $\left\langle h^{\varepsilon, \tau}\right\rangle_{R}=\langle h\rangle_{R}$, we get

$$
\mathbf{E} h\left(a^{\varepsilon}(\tau)\right)-\mathbf{E}\langle h\rangle_{R}\left(a^{\varepsilon}(\tau)\right) \rightarrow 0 \quad \text { as } \quad \varepsilon \rightarrow 0 \quad \text { uniformly in } \mathcal{C} .
$$

Jointly with (4.9) this implies the desired convergence.

\subsection{Proof of Theorem 2.4}

To prove the theorem, it suffices to find weak solutions $a^{\varepsilon}(\tau)$ and $u(\tau)$ of eq. (2.29) and the effective equation (2.31) correspondingly, defined on the same probability space and satisfying $\mathcal{D}\left(a^{\varepsilon}(0)\right)=\mathcal{D}(u(0))=\mathcal{D}\left(u_{0}\right)$, such that for every $j \in \mathcal{C}$ we have

$$
\mathbf{E} \max _{0 \leq \tau \leq T}\left|w_{j}^{\varepsilon}(\tau)\right| \rightarrow 0 \quad \text { as } \varepsilon \rightarrow 0 \quad \text { uniformly in } \mathcal{C},
$$

where $w^{\varepsilon}(\tau):=a^{\varepsilon}(\tau)-u(\tau)$. Let us start with constructing suitable Brownian motions $\beta$ and $\boldsymbol{\beta}$. Denote

$$
\boldsymbol{\beta}^{\varepsilon}=\left(\boldsymbol{\beta}_{j}^{\varepsilon}\right)_{j \in \mathcal{C}}, \quad \boldsymbol{\beta}_{j}^{\varepsilon}(\tau):=\sqrt{2} \int_{0}^{\tau} \mathrm{e}^{-i \varepsilon^{-1} s} \mathrm{~d} \beta_{j} .
$$


Proposition 4.3. We have $\mathcal{D}\left(\boldsymbol{\beta}^{\varepsilon}(\cdot)\right) \rightarrow \mathcal{D}(\boldsymbol{\beta}(\cdot))$ as $\varepsilon \rightarrow 0$ in $C\left([0, T], \mathbb{C}^{|\mathcal{C}|}\right)$.

Proof of the proposition is given after the end of the proof of the theorem. Due to Proposition 4.3 and Skorokhod theorem, we can find random processes $\widetilde{\boldsymbol{\beta}}^{\varepsilon}(\tau)$ and $\widetilde{\boldsymbol{\beta}}(\tau)$, defined on the same probability space and satisfying $\mathcal{D}\left(\widetilde{\boldsymbol{\beta}}^{\varepsilon}(\cdot)\right)=\mathcal{D}\left(\boldsymbol{\beta}^{\varepsilon}(\cdot)\right), \mathcal{D}(\widetilde{\boldsymbol{\beta}}(\cdot))=\mathcal{D}(\boldsymbol{\beta}(\cdot))$, such that

$$
\widetilde{\boldsymbol{\beta}}^{\varepsilon} \rightarrow \widetilde{\boldsymbol{\beta}} \text { as } \varepsilon \rightarrow 0 \text { in } C\left([0, T], \mathbb{C}^{|\mathcal{C}|}\right) \text { a.s. }
$$

Take solutions $a^{\varepsilon}(\tau)$ and $u(\tau)$ of Eq. (2.29) and the effective equation (2.31) on the probability space above (extended if needed), corresponding to the Brownian motions $\widetilde{\beta}(\tau):=\frac{1}{\sqrt{2}} \int_{0}^{\tau} \mathrm{e}^{i \varepsilon^{-1} s} \mathrm{~d} \widetilde{\boldsymbol{\beta}}^{\varepsilon}$ and $\widetilde{\boldsymbol{\beta}}$, and having the same initial conditions, distributed as $u_{0}$. Let us show that the process $w^{\varepsilon}=a^{\varepsilon}-u$ satisfies (4.10). Due to Eqs. (2.29) and (2.31),

$$
\begin{aligned}
\left|w_{j}^{\varepsilon}(\tau)\right| \leq & \left|\int_{0}^{\tau} \mathrm{e}^{-i \varepsilon^{-1} s} P_{j}\left(\mathrm{e}^{i \varepsilon^{-1} s} a^{\varepsilon}(s)\right)-\mathcal{R}_{j}\left(a^{\varepsilon}(s)\right) \mathrm{d} s\right| \\
& +\left|\int_{0}^{\tau} \mathcal{R}_{j}\left(a^{\varepsilon}(s)\right)-\mathcal{R}_{j}(u(s)) \mathrm{d} s\right| \\
& +\sqrt{\mathcal{T}_{j}}\left|\widetilde{\boldsymbol{\beta}}_{j}^{\varepsilon}(\tau)-\widetilde{\boldsymbol{\beta}}_{j}(\tau)\right|=: \mathcal{Y}_{1 j}^{\varepsilon}(\tau)+\mathcal{Y}_{2 j}^{\varepsilon}(\tau)+\mathcal{Y}_{3 j}^{\varepsilon}(\tau) .
\end{aligned}
$$

First, we will estimate terms $\mathcal{Y}_{1 j}^{\varepsilon}, \mathcal{Y}_{2 j}^{\varepsilon}$ and $\mathcal{Y}_{3 j}^{\varepsilon}$ separately.

Terms $\mathcal{Y}_{1 j}^{\varepsilon}$ and $\mathcal{Y}_{3 j}^{\varepsilon}$. Using that $\mathbf{E} \max _{0 \leq \tau \leq T}\left|\widetilde{\boldsymbol{\beta}}_{j}^{\varepsilon}(\tau)\right|^{2} \vee\left|\widetilde{\boldsymbol{\beta}}_{j}(\tau)\right|^{2}<C$, it is not difficult to show that (4.11) implies

$$
\mathbf{E} \max _{0 \leq \tau \leq T} \mathcal{Y}_{3 j}^{\varepsilon}(\tau) \rightarrow 0 \quad \text { as } \quad \varepsilon \rightarrow 0
$$

Since each of the vectors $\boldsymbol{\beta}^{\varepsilon}$ and $\boldsymbol{\beta}$ has independent components, the processes $\widetilde{\boldsymbol{\beta}}_{j}^{\varepsilon}, \widetilde{\boldsymbol{\beta}}_{j}$ can be chosen independently from the choice of the set $\mathcal{C}$, so that the convergence above holds uniformly in $\mathcal{C}$.

Applying Lemma 4.1 to the function $h(u, \theta)=\mathrm{e}^{-i \theta} P_{j}\left(\mathrm{e}^{i \theta} u\right)$, we have

$$
\mathbf{E} \max _{0 \leq \tau \leq T} \mathcal{Y}_{1 j}^{\varepsilon}(\tau) \rightarrow 0 \quad \text { as } \varepsilon \rightarrow 0 \text { uniformly in } \mathcal{C} \text {. }
$$

Denote $\delta^{\varepsilon}=\left(\delta_{j}^{\varepsilon}\right)_{j \in \mathcal{C}}$, where $\delta_{j}^{\varepsilon}:=\mathbf{E} \max _{0 \leq \tau \leq T}\left(\mathcal{Y}_{1 j}^{\varepsilon}(\tau)+\mathcal{Y}_{3 j}^{\varepsilon}(\tau)\right)$, so that

$$
\delta_{j}^{\varepsilon} \rightarrow 0 \quad \text { as } \varepsilon \rightarrow 0 \quad \text { uniformly in } \mathcal{C} .
$$

Term $\mathcal{Y}_{2 j}^{\varepsilon}$. Since, by assumption $H V$, the second derivatives of the potential $V$ have at most a quadratic growth, the same holds for the first derivatives of the functions $\mathcal{R}_{j}$. Then, for each $j \in \mathcal{C}$ and any $\tau \geq 0$, we have

$$
\mathcal{Y}_{2 j}^{\varepsilon} \leq C \int_{0}^{\tau}\left(1+\sum_{k:|k-j| \leq 1}\left(\left|a_{k}^{\varepsilon}\right|^{2}+\left|u_{k}\right|^{2}\right)\right) \sum_{k:|k-j| \leq 1}\left|w_{k}^{\varepsilon}\right| \mathrm{d} s .
$$


Now we go back to the proof of (4.10). Define for $R>0$ a stopping time ${ }^{22}$ $\tau_{R}=\inf \left\{\tau \geq 0: \exists m \in \mathcal{C}\right.$ satisfying $\left.\left|a_{m}^{\varepsilon}(\tau)\right|^{2} \vee\left|u_{m}(\tau)\right|^{2} \geq R\left(|m|^{1 / 2}+1\right)\right\}$

For $L \in \mathbb{N}$ denote $\left|w^{\varepsilon}\right|_{L}:=\sum_{|j| \leq L} \mathrm{e}^{-|j|}\left|w_{j}^{\varepsilon}\right|$. Since for any $s \leq \tau_{R}$ and $k \in \mathcal{C}$ satisfying $|k| \leq L+1$, we have

$$
\left|a_{k}^{\varepsilon}(s)\right|^{2} \vee\left|u_{k}(s)\right|^{2} \leq R\left((L+1)^{1 / 2}+1\right) \leq C(R) \sqrt{L},
$$

estimates (4.12) and (4.14) imply that for $0 \leq \tau \leq T$ we have

$$
\begin{aligned}
& \mathbf{E}\left|w^{\varepsilon}\left(\tau \wedge \tau_{R}\right)\right|_{L} \\
& \leq\left|\delta^{\varepsilon}\right|_{L}+C \sum_{|j| \leq L} \mathrm{e}^{-|j|} \mathbf{E} \int_{0}^{\tau \wedge \tau_{R}}\left(1+\sum_{k:|k-j| \leq 1}\left(\left|a_{k}^{\varepsilon}\right|^{2}+\left|u_{k}\right|^{2}\right)\right) \sum_{k:|k-j| \leq 1}\left|w_{k}^{\varepsilon}\right| \mathrm{d} s \\
& \leq\left|\delta^{\varepsilon}\right|_{L}+C_{1}(R) \sqrt{L} \mathbf{E} \int_{0}^{\tau \wedge \tau_{R}} \sum_{|j| \leq L} \sum_{k:|k-j| \leq 1} \mathrm{e}^{-|j|}\left|w_{k}^{\varepsilon}\right| \mathrm{d} s \\
& \leq\left|\delta^{\varepsilon}\right|_{L}+C_{2}(R) \sqrt{L} \mathbf{E} \int_{0}^{\tau \wedge \tau_{R}}\left(\left|w^{\varepsilon}\right|_{L}+\mathrm{e}^{-L} \sum_{|k|=L+1}\left|w_{k}^{\varepsilon}\right|\right) \mathrm{d} s \\
& \left.\leq\left|\delta^{\varepsilon}\right|_{L}+C_{2}(R) \sqrt{L} \int_{0}^{\tau} \int_{|k|=L+1}\left|w_{k}^{\varepsilon}\left(s \wedge \tau_{R}\right)\right|\right) \mathrm{d} s .
\end{aligned}
$$

Due to (4.16), for $k \in \mathcal{C}$ satisfying $|k|=L+1$, we have $\left|w_{k}^{\varepsilon}\left(s \wedge \tau_{R}\right)\right| \leq \mid a_{k}^{\varepsilon}(s \wedge$ $\left.\tau_{R}\right)|+| u_{k}\left(s \wedge \tau_{R}\right) \mid \leq C(R) L^{1 / 4}$. Since the cardinality of the set $\{k:|k|=L+1\}$ is bounded by $C L^{d-1}$, we get

$$
\sum_{|k|=L+1}\left|w_{k}^{\varepsilon}\left(s \wedge \tau_{R}\right)\right| \leq C(R) L^{d-3 / 4} .
$$

By the Gronwall lemma, relation (4.17) joined with the inequality above implies

$$
\mathbf{E}\left|w^{\varepsilon}\left(\tau \wedge \tau_{R}\right)\right|_{L} \leq\left(\left|\delta^{\varepsilon}\right|_{L}+C(R) \mathrm{e}^{-L} L^{d-3 / 4}\right) \mathrm{e}^{C(R) \sqrt{L} \tau} .
$$

In view of estimate (4.13), we have $\left|\delta^{\varepsilon}\right|_{L} \rightarrow 0$ as $\varepsilon \rightarrow 0$ uniformly in $\mathcal{C}$, for each $L \in \mathbb{N}$. Letting $L \rightarrow \infty$ and $\varepsilon \rightarrow 0$ in such a way that $\left|\delta^{\varepsilon}\right|{ }_{L} \mathrm{e}^{C(R) \sqrt{L} \tau} \rightarrow 0$, we obtain that $\mathbf{E}\left|w^{\varepsilon}\left(\tau \wedge \tau_{R}\right)\right|_{L} \rightarrow 0$ uniformly in $\mathcal{C}$. In particular, for each $k \in \mathcal{C}$ and any $0 \leq \tau \leq T$ we have

$$
\mathbf{E}\left|w_{k}^{\varepsilon}\left(\tau \wedge \tau_{R}\right)\right| \rightarrow 0 \quad \text { as } \varepsilon \rightarrow 0 \quad \text { uniformly in } \mathcal{C} .
$$

\footnotetext{
${ }^{22}$ We cannot define a stopping time in a standard way $\hat{\tau}_{R}=\inf \left\{\tau \geq 0: \max _{m \in \mathcal{C}}\left|a_{m}^{\varepsilon}(\tau)\right|^{2} \vee\right.$ $\left.\left|u_{m}(\tau)\right|^{2} \geq R\right\}$ since $\mathbf{P}\left(\hat{\tau}_{R} \leq T\right) \rightarrow 0$ as $R \rightarrow \infty$ non-uniformly in $\mathcal{C}$. To overcome this difficulty, we introduce a stopping time $\tau_{R}$ which admits a slow growth of the absolute values $\left|a_{m}^{\varepsilon}\right|,\left|u_{m}\right|$ with respect to $|m|$.
} 
Inequality (4.12) jointly with estimate (4.14) implies

$$
\mathbf{E} \max _{0 \leq \tau \leq T}\left|w_{j}^{\varepsilon}\left(\tau \wedge \tau_{R}\right)\right| \leq \delta_{j}^{\varepsilon}+C(R)(\sqrt{|j|}+1) \int_{0}^{T} \mathbf{E} \sum_{k:|k-j| \leq 1}\left|w_{k}^{\varepsilon}\left(s \wedge \tau_{R}\right)\right| \mathrm{d} s .
$$

Since the function under the time integral in (4.19) is bounded by a constant $C(R, j)$, in view of (4.18) the dominated convergence theorem implies that this integral tends to zero as $\varepsilon \rightarrow 0$, uniformly in $\mathcal{C}$. Then, in view of (4.13) for every $j \in \mathcal{C}$, we have

$$
\mathbf{E} \max _{0 \leq \tau \leq T}\left|w_{j}^{\varepsilon}\left(\tau \wedge \tau_{R}\right)\right| \rightarrow 0 \quad \text { as } \quad \varepsilon \rightarrow 0 \quad \text { uniformly in } \mathcal{C} \text {. }
$$

To recover convergence (4.10) from (4.20), we will need the following proposition.

Proposition 4.4. (i) $\mathbf{P}\left(\tau_{R} \leq T\right) \rightarrow 0$ as $R \rightarrow \infty$ uniformly in $\mathcal{C}$.

(ii) For all $j \in \mathcal{C}$, we have $\mathbf{E} \max _{0 \leq \tau \leq T} \mathrm{e}^{\alpha\left|u_{j}(\tau)\right|^{2}} \leq C$, where $\alpha, C>0$ are the constants from Lemma 2.1 .

Using the Cauchy-Schwarz inequality, we find

$$
\begin{aligned}
\mathbf{E} & \max _{0 \leq \tau \leq T}\left|w_{j}^{\varepsilon}(\tau)\right| \\
& =\mathbf{E} \mathbb{I}_{\left(\tau_{R} \geq T\right)} \max _{0 \leq \tau \leq T}\left|w_{j}^{\varepsilon}\left(\tau \wedge \tau_{R}\right)\right|+\mathbf{E} \mathbb{I}_{\left(\tau_{R} \leq T\right)} \max _{0 \leq \tau \leq T}\left|w_{j}^{\varepsilon}(\tau)\right| \\
& \leq \mathbf{E} \max _{0 \leq \tau \leq T}\left|w_{j}^{\varepsilon}\left(\tau \wedge \tau_{R}\right)\right|+\left(\mathbf{P}\left(\tau_{R} \leq T\right)\right)^{1 / 2}\left(\mathbf{E} \max _{0 \leq \tau \leq T}\left|w_{j}^{\varepsilon}(\tau)\right|^{2}\right)^{1 / 2} .
\end{aligned}
$$

Proposition 4.4.ii joined with the estimate of Lemma 2.1.i implies that $\mathbf{E} \max _{0 \leq \tau \leq T}\left|w_{j}^{\varepsilon}(\tau)\right|^{2}<C$. Then, in view of (4.20) and Proposition 4.4.i, we arrive at (4.10).

Proof of Proposition 4.4. (i) We have

$$
\begin{aligned}
\mathbf{P}\left(\tau_{R} \leq T\right)= & \mathbf{P}\left(\exists j \in \mathcal{C}: \max _{0 \leq \tau \leq T}\left|a_{j}^{\varepsilon}(\tau)\right|^{2} \vee\left|u_{j}(\tau)\right|^{2} \geq R\left(|j|^{1 / 2}+1\right)\right) \\
= & \mathbf{P}\left(\exists j \in \mathcal{C}: \max _{0 \leq \tau \leq T}\left|a_{j}^{\varepsilon}\left(\tau \wedge \tau_{R}\right)\right|^{2} \vee\left|u_{j}\left(\tau \wedge \tau_{R}\right)\right|^{2} \geq R\left(|j|^{1 / 2}+1\right)\right) \\
\leq & \sum_{j \in \mathcal{C}} \mathbf{P}\left(\max _{0 \leq \tau \leq T}\left|a_{j}^{\varepsilon}\left(\tau \wedge \tau_{R}\right)\right|^{2} \geq R\left(|j|^{1 / 2}+1\right)\right) \\
& +\sum_{j \in \mathcal{C}} \mathbf{P}\left(\max _{0 \leq \tau \leq T}\left|u_{j}\left(\tau \wedge \tau_{R}\right)\right|^{2} \geq R\left(|j|^{1 / 2}+1\right)\right) .
\end{aligned}
$$

In view of the estimate $\mathbf{E} \max _{0 \leq \tau \leq T} \mathrm{e}^{\alpha\left|a_{j}^{\varepsilon}\left(\tau \wedge \tau_{R}\right)\right|^{2}} \leq \mathbf{E} \max _{0 \leq \tau \leq T} \mathrm{e}^{\alpha\left|a_{j}^{\varepsilon}(\tau)\right|^{2}}<$ $C$ provided by Lemma 2.1, convergence (4.20) joined with the Fatou lemma implies

$$
\mathbf{E} \max _{0 \leq \tau \leq T} \mathrm{e}^{\alpha\left|u_{j}\left(\tau \wedge \tau_{R}\right)\right|^{2}} \leq C, \quad j \in \mathcal{C} .
$$


Then, using the Chebyshev inequality, we obtain

$$
\begin{aligned}
\mathbf{P}\left(\tau_{R} \leq T\right) \leq C \sum_{j \in \mathcal{C}} \mathrm{e}^{-\alpha R\left(|j|^{1 / 2}+1\right)} \leq C \sum_{j \in \mathbb{Z}^{d}} \mathrm{e}^{-\alpha R\left(|j|^{1 / 2}+1\right)} & \rightarrow 0 \quad \text { as } R \rightarrow \infty \\
& \text { uniformly in } \mathcal{C}
\end{aligned}
$$

(ii) The desired estimate follows from item (i) joined with (4.21) and the Fatou lemma.

Proof of Proposition 4.3. Since components of each of the vectors $\boldsymbol{\beta}$ and $\boldsymbol{\beta}^{\varepsilon}$ are independent, it suffices to prove that $\mathcal{D}\left(\boldsymbol{\beta}_{j}^{\varepsilon}(\cdot)\right) \rightarrow \mathcal{D}\left(\boldsymbol{\beta}_{j}(\cdot)\right)$ as $\varepsilon \rightarrow 0$ in $C([0, T], \mathbb{C})$ for each $j \in \mathcal{C}$. In a standard way, it can be shown that the set of measures $\left\{\mathcal{D}\left(\boldsymbol{\beta}_{j}^{\varepsilon}(\cdot)\right), 0<\varepsilon \leq 1\right\}$ is tight. Let $Q$ be its limiting point as $\varepsilon_{k} \rightarrow 0$. Take a process $\hat{\boldsymbol{\beta}}_{j}(\tau)$ such that $\mathcal{D}\left(\hat{\boldsymbol{\beta}}_{j}(\cdot)\right)=Q$. We need to show that $\hat{\boldsymbol{\beta}}_{j}$ is a standard complex Brownian motion. Since the processes $\boldsymbol{\beta}_{j}^{\varepsilon}$ are uniformly in $\varepsilon$ square-integrable martingales, the process $\hat{\boldsymbol{\beta}}_{j}$ also is. Then, it suffices to establish that

$$
\left[\hat{\beta}_{1}\right]_{\tau}=\left[\hat{\beta}_{2}\right]_{\tau}=\tau \text { and }\left[\hat{\beta}_{1}, \hat{\beta}_{2}\right]_{\tau} \equiv 0,
$$

where $\hat{\boldsymbol{\beta}}_{1}=\operatorname{Re} \hat{\boldsymbol{\beta}}_{j}, \hat{\boldsymbol{\beta}}_{2}=\operatorname{Im} \hat{\boldsymbol{\beta}}_{j},[\cdot]_{\tau}$ denotes the quadratic variation and $[\cdot, \cdot]_{\tau}$ stands for the cross-variation. We will only show that $\left[\hat{\beta}_{1}\right]_{\tau}=\tau$, proofs of the other assertions are similar. Due to the definition of the quadratic variation, for this purpose it suffices to prove that the process $\gamma(\tau):=\left(\hat{\beta}_{1}(\tau)\right)^{2}-\tau$ is a martingale. Denote $\gamma^{\varepsilon_{k}}(\tau):=\left(\beta_{1}^{\varepsilon_{k}}(\tau)\right)^{2}-\tau$, where $\beta_{1}^{\varepsilon_{k}}:=\operatorname{Re} \boldsymbol{\beta}_{j}^{\varepsilon_{k}}$. Clearly,

$$
\mathcal{D}\left(\gamma^{\varepsilon_{k}}(\cdot)\right) \rightarrow \mathcal{D}(\gamma(\cdot)) \quad \text { as } k \rightarrow \infty .
$$

On the other hand, since $\left[\beta_{1}^{\varepsilon_{k}}\right]_{\tau}=2 \int_{0}^{\tau} \cos ^{2}\left(\varepsilon_{k}^{-1} s\right) \mathrm{d} s$,

$$
\gamma^{\varepsilon_{k}}(\tau)=M^{\varepsilon_{k}}(\tau)+2 \int_{0}^{\tau} \cos ^{2}\left(\varepsilon_{k}^{-1} s\right) \mathrm{d} s-\tau,
$$

where $M^{\varepsilon_{k}}(\tau)$ is a square-integrable martingale. Since $\max _{0 \leq \tau \leq T}\left|2 \int_{0}^{\tau} \cos ^{2}\left(\varepsilon_{k}^{-1} s\right) d s-\tau\right| \rightarrow 0$ as $k \rightarrow \infty$, (4.23) jointly with (4.22) implies

$$
\mathcal{D}\left(M^{\varepsilon_{k}}(\cdot)\right) \rightarrow \mathcal{D}(\gamma(\cdot)) \quad \text { as } k \rightarrow \infty,
$$

so that $\gamma(\tau)$ is a square-integrable martingale as well.

\subsection{Proof of Theorem 2.8}

Item (i). Let $u^{\varepsilon}(\tau)$ and $u(\tau)$ be stationary solutions of Eq. (2.25) and the effective equation (2.31) correspondingly, $\mathcal{D}\left(u^{\varepsilon}(\tau)\right) \equiv \mu^{\varepsilon}$ and $\mathcal{D}(u(\tau)) \equiv \mu$. Take a function $h \in \mathcal{L}_{b}\left(\mathbb{C}^{|\mathcal{C}|}\right)$. It suffices to show that

$$
\mathbf{E} h\left(u^{\varepsilon}(\tau)\right) \rightarrow \mathbf{E} h(u(\tau)) \text { as } \varepsilon \rightarrow 0 \text {. }
$$


Let us pass to the $a$-variables $a^{\varepsilon}(\tau)$, corresponding to the process $u^{\varepsilon}(\tau)$. Consider the space $C\left([0, \infty), \mathbb{C}^{|\mathcal{C}|}\right)$ provided with the topology of uniform convergence on finite time intervals, which is given by the metrics

$$
\rho(v, w)=\sum_{K=1}^{\infty} 2^{-K} \sup _{0 \leq \tau \leq K}|v(\tau)-w(\tau)| \wedge 1 .
$$

Proposition 4.5. The set of laws $\mathcal{D}\left(a^{\varepsilon}(\cdot)\right), 0<\varepsilon<\varepsilon_{0},{ }^{23}$ is tight in $C\left([0, \infty), \mathbb{C}^{|\mathcal{C}|}\right)$.

Proof. It suffices to establish that the set of measures $\mathcal{D}\left(a^{\varepsilon}(\cdot)\right)$ is tight in $C\left([0, T], \mathbb{C}^{|\mathcal{C}|}\right)$ for any $T \geq 0$. The latter follows in a standard way from the estimates of Lemma 2.1, Eq. (2.29) and Arzela-Ascoli theorem.

Consider some limiting point

$$
\mathcal{D}\left(a^{\varepsilon_{k}}(\cdot)\right) \rightarrow Q \quad \text { as } \quad \varepsilon_{k} \rightarrow 0 .
$$

It turns out that the measure $Q$ is a law of a weak solution of the effective equation:

Proposition 4.6. The measure $Q$ coincides with the law $\mathcal{D}\left(u^{0}(\cdot)\right)$ in the space $C\left([0, \infty), \mathbb{C}^{|\mathcal{C}|}\right)$, where $u^{0}(\tau)$ is a weak solution of the effective equation $(2.31)$.

Proposition 4.6 can be established by argument similar to that used in the proof of Theorem 2.4. ${ }^{24}$ We do not prove it here since below we will establish an analogous result in a more complicated, infinite-dimensional setting (see Proposition 4.8). Proposition 4.6 provides that for any $\tau \geq 0$ we have $\mathbf{E} h\left(a^{\varepsilon_{k}}(\tau)\right) \rightarrow \mathbf{E} h\left(u^{0}(\tau)\right)$ as $\varepsilon_{k} \rightarrow 0$. Jointly with Corollary 4.2 this implies

$$
\mathbf{E} h\left(u^{\varepsilon_{k}}(\tau)\right) \rightarrow \mathbf{E} h\left(u^{0}(\tau)\right) \text { as } \varepsilon_{k} \rightarrow 0 .
$$

Since the process $u^{\varepsilon}$ is stationary, (4.27) implies that the process $u^{0}(\tau)$ also is, so that $\mathcal{D}\left(u^{0}(\tau)\right) \equiv \mathcal{D}(u(\tau)) \equiv \mu$. Thus, we get (4.24).

Item (ii). Assume that condition $H_{\infty}$ is satisfied. Recall that the uniformity in $\mathcal{C}$ of the weak convergences of measures through all the text is understood in the sense of finite-dimensional projections. That is, we need to prove that for any bounded set $\Lambda \subset \mathbb{Z}^{d}$ and a function $h \in \mathcal{L}_{b}\left(\mathbb{C}^{|\mathcal{C}|}\right)$ satisfying $\operatorname{Supp} h \subseteq \Lambda$, convergence (4.24) holds uniformly in $\mathcal{C}$ satisfying $\mathcal{C} \supseteq \Lambda$. For this purpose, it suffices to show that for any sequence of bounded sets $\left(\mathcal{C}_{n}\right)_{n \in \mathbb{N}}, \mathcal{C}_{n} \subset \mathbb{Z}^{d}$ satisfying $\mathcal{C}_{n} \supseteq \Lambda$ for each $n \in \mathbb{N}$, there exists a subsequence $\left(\hat{\mathcal{C}}_{n}\right)_{n \in \mathbb{N}}$ such that the desired convergence holds uniformly in $\mathcal{C} \in\left(\hat{\mathcal{C}}_{n}\right)_{n \in \mathbb{N}}$.

The proof follows a scheme below. As $\left(\hat{\mathcal{C}}_{n}\right)_{n \in \mathbb{N}}$, we take an arbitrary subsequence which has a "limit" as $n \rightarrow \infty$, say $\hat{\mathcal{C}}_{\infty}$. Ass. $H \mathcal{C}_{\infty}$ provides that the corresponding $\hat{\mathcal{C}}_{\infty}$-effective equation has a unique stationary measure in

${ }^{23}$ Recall that $\varepsilon_{0}$ is defined in Lemma 2.1.

${ }^{24}$ Note that this argument does not lead to the uniformity in $\mathcal{C}$ of convergence (4.26). Indeed, in the present case the distributions of initial conditions $\mathcal{D}\left(a^{\varepsilon_{k}}(0)\right)$ are different for different $\varepsilon_{k}$, so we should add in (4.17) the term $\mathbf{E}\left|w^{\varepsilon_{k}}(0)\right|_{L}$. We do not know if it convergences to zero as $\varepsilon_{k} \rightarrow 0$ uniformly in $\mathcal{C}$. 
$\mathcal{M}^{\hat{\mathcal{C}}_{\infty}}$. Then, arguing as in item (i), we establish convergence (4.32), where $u^{\varepsilon, \hat{\mathcal{C}}_{n}}$ denotes a stationary solution of Eq. $(2.25)$ with $\mathcal{C}=\hat{\mathcal{C}}_{n}$, while $u^{\infty}$ denotes that of the $\hat{\mathcal{C}}_{\infty}$-effective equation. This provides control of the expectations $\mathbf{E} h\left(u^{\varepsilon, \hat{\mathcal{C}}_{n}}(\tau)\right)$ for large $n$ and small $\varepsilon$, sufficient to prove the desired uniformity of convergence. Now let us present the rigorous proof. Denote by $B_{N}$ a ball in $\left(\mathbb{Z}^{d},|\cdot|\right)$ of the radius $N$ centred at zero, where $|\cdot|$ is the $l_{1}$-norm in $\mathbb{Z}^{d}$.

Proposition 4.7. Let $\left(\mathcal{C}_{n}\right)_{n \in \mathbb{N}}$ be a sequence of bounded sets in $\mathbb{Z}^{d}$. Then, there exists a subsequence $\left(\hat{\mathcal{C}}_{n}\right)_{n \in \mathbb{N}}$ and a set $\hat{\mathcal{C}}_{\infty} \subseteq \mathbb{Z}^{d}$ such that $\hat{\mathcal{C}}_{n} \rightarrow \hat{\mathcal{C}}_{\infty}$ as $n \rightarrow \infty$ in the sense that for any $N \in \mathbb{N}$ and all $n \geq N$ we have $\hat{\mathcal{C}}_{n} \cap B_{N}=\hat{\mathcal{C}}_{\infty} \cap B_{N}$.

Proof. Since the number of sites in $B_{1}$ is finite, there exists a subsequence $\left(\mathcal{C}_{n_{n_{1}}}\right)_{n_{1} \in \mathbb{N}}$ such that for every $j \in B_{1}$ we have either $j \in \mathcal{C}_{n_{n_{1}}}$ or $j \notin \mathcal{C}_{n_{n_{1}}}$ at the same time for all $n_{1}$. Then, the set $\mathcal{C}_{n_{n_{1}}} \cap B_{1}$ is independent from $n_{1}$. Similarly, we find a subsequence $\left(\mathcal{C}_{n_{n_{1_{n}}}}\right)_{n_{2} \in \mathbb{N}}$ such that the set $\mathcal{C}_{n_{n_{1} n_{2}}} \cap B_{2}$ is independent from $n_{2}$. We continue the procedure and apply the diagonal process to choose a subsequence $(\hat{\mathcal{C}})_{n \in \mathbb{N}}=\mathcal{C}_{n_{1}}, \mathcal{C}_{n_{n_{12}}}, \mathcal{C}_{n_{n_{1} n_{23}}}, \ldots$ Then for any $N \in \mathbb{N}$ the sets $\hat{\mathcal{C}}_{n} \cap B_{N}$ coincide for all $n \geq N$. Let $\hat{\mathcal{C}}_{\infty}:=\cup_{n \in \mathbb{N}}\left(\hat{\mathcal{C}}_{n} \cap B_{n}\right)$. Clearly, the pair $\left(\hat{\mathcal{C}}_{n}\right)_{n \in \mathbb{N}}$ and $\hat{\mathcal{C}}_{\infty}$ satisfies the assertion of the proposition.

Choose a subsequence $\left(\hat{\mathcal{C}}_{n}\right)_{n \in \mathbb{N}}$ of the sequence $\left(\mathcal{C}_{n}\right)_{n \in \mathbb{N}}$ as in Proposition 4.7. Let $u^{\varepsilon, \hat{\mathcal{C}}_{n}}(\tau)$ be a stationary solution of Eq. (2.25) with $\mathcal{C}=\hat{\mathcal{C}}_{n}$, and $u^{\hat{\mathcal{C}}_{n}}(\tau)$ be that of the effective equation (2.31). We need to prove that convergence (4.24) holds uniformly in $\mathcal{C} \in\left(\hat{\mathcal{C}}_{n}\right)_{n \in \mathbb{N}}$, i.e. to show that for any $\delta>0$ there exists $\hat{\varepsilon}>0$ such that for any $\varepsilon<\hat{\varepsilon}$ and all $n \in \mathbb{N}$ we have

$$
\left|\mathbf{E} h\left(u^{\varepsilon, \hat{\mathcal{C}}_{n}}(\tau)\right)-\mathbf{E} h\left(u^{\hat{\mathcal{C}}_{n}}(\tau)\right)\right|<\delta .
$$

Denote $u^{\varepsilon, n}=\left(u_{j}^{\varepsilon, n}\right)_{j \in \hat{\mathcal{C}}_{\infty}}$, where

$$
u_{j}^{\varepsilon, n}:=\left\{\begin{array}{cl}
u_{j}^{\varepsilon, \hat{\mathcal{C}}_{n}}, & \text { if } j \in \hat{\mathcal{C}}_{\infty} \cap \hat{\mathcal{C}}_{n}, \\
0, & \text { if } j \in \hat{\mathcal{C}}_{\infty} \backslash \hat{\mathcal{C}}_{n} .
\end{array}\right.
$$

Let $a^{\varepsilon, n}=\left(a_{j}^{\varepsilon, n}\right)_{j \in \hat{\mathcal{C}}_{\infty}}$ be the corresponding $a$-variables. Consider the space $C\left([0, \infty), \mathbb{C}^{\left|\hat{\mathcal{C}}_{\infty}\right|}\right)$ provided with the topology of uniform convergence on finite time intervals which is given by the metrics (4.25), where the distance $\mid v(\tau)-$ $w(\tau) \mid$ is replaced by

$$
\rho_{\mathbb{C}^{\left|\hat{\mathcal{C}}_{\infty}\right|}}(v(\tau), w(\tau))=\sum_{j \in \hat{\mathcal{C}}_{\infty}} 2^{-|j|}\left|v_{j}(\tau)-w_{j}(\tau)\right| \wedge 1 .
$$

Using the uniformity in $\mathcal{C}$ of estimates from Lemma 2.1 we get that the set of laws $\left\{\mathcal{D}\left(a^{\varepsilon, n}(\cdot)\right), 0<\varepsilon \leq 1, n \in \mathbb{N}\right\}$ is tight in the space $C\left([0, \infty), \mathbb{C}^{\left|\hat{\mathcal{C}}_{\infty}\right|}\right)$. Let $Q^{\infty}$ be its limiting point as $\varepsilon_{k} \rightarrow 0, n_{k} \rightarrow \infty$.

Proposition 4.8. In the space $C\left([0, \infty), \mathbb{C}^{\left|\hat{\mathcal{C}}_{\infty}\right|}\right)$ the measure $Q^{\infty}$ coincides with the law $\mathcal{D}\left(u^{\infty}(\cdot)\right)$, where $u^{\infty}(\tau)$ is a weak solution of the $\hat{\mathcal{C}}_{\infty}$-effective equation. 
For any $j \in \hat{\mathcal{C}}_{\infty}, 0<\lambda \leq 1$ and $\tau \geq 0$, it satisfies

$$
\mathbf{E} \max _{s \in[\tau, \tau+1]} \mathrm{e}^{\alpha\left|u_{j}^{\infty}(s)\right|} \leq C .
$$

Before presenting the proof of the proposition, let us finish the proof of the theorem. Since $\Lambda \subseteq \hat{\mathcal{C}}_{n}$ for all $n \in \mathbb{N}$, we have $\Lambda \subseteq \hat{\mathcal{C}}_{\infty}$. Then, due to the inclusion Supp $h \subseteq \Lambda$, the function $h$ can be considered as $h: \mathbb{C}^{\left|\hat{\mathcal{C}}_{\infty}\right|} \mapsto \mathbb{R}$. Proposition 4.8 implies that $\mathbf{E} h\left(a^{\varepsilon_{k}, n_{k}}(\tau)\right) \rightarrow \mathbf{E} h\left(u^{\infty}(\tau)\right)$ as $\varepsilon_{k} \rightarrow 0, n_{k} \rightarrow \infty$. Since, due to Corollary 4.2, we have $\mathbf{E} h\left(a^{\varepsilon_{k}, n_{k}}(\tau)\right)-\mathbf{E} h\left(u^{\varepsilon_{k}, n_{k}}(\tau)\right) \rightarrow 0$ as $\varepsilon_{k} \rightarrow 0, n_{k} \rightarrow \infty$, we get

$$
\mathbf{E} h\left(u^{\varepsilon_{k}, n_{k}}(\tau)\right) \rightarrow \mathbf{E} h\left(u^{\infty}(\tau)\right) \quad \text { as } \quad \varepsilon_{k} \rightarrow 0, n_{k} \rightarrow \infty,
$$

so that $u^{\infty}$ is a stationary process. Due to (4.30), the stationary distribution $\mathcal{D}\left(u^{\infty}(\tau)\right)$ belongs to $\mathcal{M}^{\hat{\mathcal{C}}_{\infty}}$. Then, in view of ass. $H \mathcal{C}_{\infty}$, it is defined uniquely, so that the limit in (4.31) does not depend on the sequence $\left(\varepsilon_{k}, n_{k}\right)_{k \in \mathbb{N}}$ and holds as $\varepsilon \rightarrow 0, n \rightarrow \infty$. Since $\operatorname{Supp} h \subseteq \Lambda \subseteq \hat{\mathcal{C}}_{\infty} \cap \hat{\mathcal{C}}_{n}$ for all $n \in \mathbb{N}$, we have $h\left(u^{\varepsilon, n}(\tau)\right) \equiv h\left(u^{\varepsilon, \hat{\mathcal{C}}_{n}}(\tau)\right)$, so that

$$
\mathbf{E} h\left(u^{\varepsilon, \hat{\mathcal{C}}_{n}}(\tau)\right) \rightarrow \mathbf{E} h\left(u^{\infty}(\tau)\right) \quad \text { as } \varepsilon \rightarrow 0, n \rightarrow \infty .
$$

Due to item $(i)$ of the theorem, for each $n \in \mathbb{N}$, we also have

$$
\mathbf{E} h\left(u^{\varepsilon, \hat{\mathcal{C}}_{n}}(\tau)\right) \rightarrow \mathbf{E} h\left(u^{\hat{\mathcal{C}}_{n}}(\tau)\right) \quad \text { as } \varepsilon \rightarrow 0 .
$$

Jointly with (4.32), this implies that $\mathbf{E} h\left(u^{\hat{\mathcal{C}}_{n}}(\tau)\right) \rightarrow \mathbf{E} h\left(u^{\infty}(\tau)\right)$ when $n \rightarrow \infty$. Consequently, for any $\delta>0$ there exist $N \in \mathbb{N}$ and $\varepsilon_{1}>0$, such that for every $n \geq N$ and $0<\varepsilon<\varepsilon_{1}$, we have

$$
\left|\mathbf{E} h\left(u^{\varepsilon, \hat{\mathcal{C}}_{n}}(\tau)\right)-\mathbf{E} h\left(u^{\infty}(\tau)\right)\right|,\left|\mathbf{E} h\left(u^{\hat{\mathcal{C}}_{n}}(\tau)\right)-\mathbf{E} h\left(u^{\infty}(\tau)\right)\right|<\delta / 2 .
$$

Then for $n$ and $\varepsilon$ as above, we get (4.28). In view of (4.33), we can choose $\varepsilon_{2}>0$ such that (4.28) is also satisfied for every $0<\varepsilon<\varepsilon_{2}$ and $n<N$. Then, it holds for all $n \in \mathbb{N}$ and $\varepsilon<\varepsilon_{1} \wedge \varepsilon_{2}$.

Proof of Proposition 4.8. Before starting the proof let us make the following remark. Since ${ }^{25} \operatorname{Supp} P_{j}$, Supp $R_{j} \subseteq\left\{k \in \mathbb{Z}^{d}:|k-j| \leq 1\right\} \cap\{k \in \mathcal{C}\}$, the functions $P_{j}$ and $\mathcal{R}_{j}$ depend on the choice of the set $\mathcal{C}$. To indicate this, we denote those corresponding to the set $\hat{\mathcal{C}}_{n_{k}}$ as $P_{j}^{k}, \mathcal{R}_{j}^{k}$ and those corresponding to the set $\hat{\mathcal{C}}_{\infty}$ as $P_{j}^{\infty}, \mathcal{R}_{j}^{\infty}$. Now, we start the proof. For shortness, we write $a^{k}:=a^{\varepsilon_{k}, n_{k}}$. Consider the process $b^{k}=\left(b_{j}^{k}\right)_{j \in \hat{\mathcal{C}}_{\infty}}$, where

$$
b_{j}^{k}(\tau)=a_{j}^{k}(\tau)-a_{j}^{k}(0)-\int_{0}^{\tau} \mathcal{R}_{j}^{\infty}\left(a^{k}(s)\right) \mathrm{d} s, \quad j \in \hat{\mathcal{C}}_{\infty} .
$$

${ }^{25}$ We recall that the functions $P_{j}$ and $\mathcal{R}_{j}$ are defined in (2.26) and (2.32) correspondingly. 
Consider also a process $u^{\infty}(\tau)$ satisfying $\mathcal{D}\left(u^{\infty}(\cdot)\right)=Q^{\infty}$, and put $b=$ $\left(b_{j}\right)_{j \in \hat{\mathcal{C}}_{\infty}}$, where

$$
b_{j}(\tau)=u_{j}^{\infty}(\tau)-u_{j}^{\infty}(0)-\int_{0}^{\tau} \mathcal{R}_{j}^{\infty}\left(u^{\infty}(s)\right) \mathrm{d} s, \quad j \in \hat{\mathcal{C}}_{\infty} .
$$

The convergence $\mathcal{D}\left(a^{k}(\cdot)\right) \rightarrow Q^{\infty}$ as $k \rightarrow \infty$ implies

$$
\mathcal{D}\left(b^{k}(\cdot)\right) \rightarrow \mathcal{D}(b(\cdot)) \quad \text { as } k \rightarrow \infty \quad \text { on } C\left([0, \infty), \mathbb{C}^{\left|\hat{\mathcal{C}}_{\infty}\right|}\right) .
$$

On the other hand, according to (4.29) and Eq. (2.29), for $j \in \hat{\mathcal{C}}_{\infty} \cap \hat{\mathcal{C}}_{n_{k}}$, we have

$$
b_{j}^{k}(\tau)=\Theta_{j}^{k}(\tau)+\hat{\boldsymbol{\beta}}_{j}^{k}(\tau),
$$

where $\hat{\boldsymbol{\beta}}^{k}=\left(\hat{\boldsymbol{\beta}}_{j}^{k}\right)_{j \in \hat{\mathcal{C}}_{\infty}}, \hat{\boldsymbol{\beta}}_{j}^{k}(\tau):=\sqrt{2 \mathcal{T}_{j}} \int_{0}^{\tau} \mathrm{e}^{-i \varepsilon_{k}^{-1} s} \mathrm{~d} \beta_{j},\left(\beta_{j}\right)_{j \in \hat{\mathcal{C}}_{\infty}}$ are standard real independent Brownian motions, and

$$
\Theta_{j}^{k}(\tau)=\int_{0}^{\tau}\left(\mathrm{e}^{-i \varepsilon_{k}^{-1} s} P_{j}^{k}\left(\mathrm{e}^{i \varepsilon_{k}^{-1} s} a^{\varepsilon_{k}, \hat{\mathcal{C}}_{n_{k}}}(s)\right)-\mathcal{R}_{j}^{\infty}\left(a^{k}(s)\right)\right) \mathrm{d} s,
$$

where $a^{\varepsilon_{k}, \hat{\mathcal{C}}_{n_{k}}}$ denotes the $a$-variables corresponding to the process $u^{\varepsilon_{k}, \hat{\mathcal{C}}_{n_{k}}}$. Since $\hat{\mathcal{C}}_{n_{k}} \rightarrow \hat{\mathcal{C}}_{\infty}$ as $k \rightarrow \infty$, for sufficiently large $k$ (depending on $j$ ), we have $P_{j}^{k}=P_{j}^{\infty}$ and $\mathcal{R}_{j}^{\infty}\left(a^{k}(s)\right) \equiv \mathcal{R}_{j}^{\infty}\left(a^{\varepsilon_{k}, \hat{\mathcal{C}}_{n_{k}}}(s)\right)$. Then, applying Lemma 4.1 to the function $h(u, \theta)=\mathrm{e}^{-i \theta} P_{j}^{\infty}\left(\mathrm{e}^{i \theta} u\right)$, we get

$$
\mathbf{E} \max _{0 \leq \tau \leq T}\left|\Theta_{j}^{k}(\tau)\right| \rightarrow 0 \quad \text { as } \quad k \rightarrow \infty \quad \text { for every } T \geq 0 .
$$

Moreover, Proposition 4.3 implies that $\mathcal{D}\left(\hat{\boldsymbol{\beta}}^{k}(\cdot)\right) \rightarrow \mathcal{D}(\hat{\boldsymbol{\beta}}(\cdot))$ as $k \rightarrow \infty$ in $C\left([0, \infty), \mathbb{C}^{\left|\hat{\mathcal{C}}_{\infty}\right|}\right)$, where $\hat{\boldsymbol{\beta}}=\left(\hat{\boldsymbol{\beta}}_{j}\right)_{j \in \hat{\mathcal{C}}_{\infty}}, \hat{\boldsymbol{\beta}}_{j}=\sqrt{\mathcal{T}_{j}} \boldsymbol{\beta}_{j}$, and $\left(\boldsymbol{\beta}_{j}\right)_{j \in \hat{\mathcal{C}}_{\infty}}$ are standard complex independent Brownian motions. Then, from (4.35), we obtain

$$
\mathcal{D}\left(b^{k}(\cdot)\right) \rightarrow \mathcal{D}(\hat{\boldsymbol{\beta}}(\cdot)) \quad \text { as } k \rightarrow \infty \quad \text { on } C\left([0, \infty), \mathbb{C}^{\left|\hat{\mathcal{C}}_{\infty}\right|}\right) .
$$

Thus, due to (4.34), we have $\mathcal{D}(b(\cdot))=\mathcal{D}(\hat{\boldsymbol{\beta}}(\cdot))$, so that $u^{\infty}(\tau)$ is a weak solution of the $\hat{\mathcal{C}}_{\infty}$-effective equation. Estimate (4.30) follows from the Fatou lemma and Lemma 2.1.

\subsection{The $\mathcal{C}_{\infty}$-Effective Equation}

In this section, we prove that under conditions of Proposition 2.7, the $\mathcal{C}_{\infty^{-}}$ effective equation defines a Markov process and is mixing, in a suitable space. In particular, this will imply Proposition 2.7. Denote

$$
\|u\|_{0}^{2}:=\sum_{k \in \widetilde{\mathcal{C}}} \gamma^{|k|}\left|u_{k}\right|^{2}
$$

where $u \in \mathbb{C}^{|\widetilde{\mathcal{C}}|}$ and $\widetilde{\mathcal{C}}=\mathcal{C}$ or $\widetilde{\mathcal{C}}=\mathcal{C}_{\infty}$ (the choice will be clear from the context). Fix the constant $1 / 2<\gamma<1$ as in Lemma 2.3. Consider the space

$$
\mathcal{X}:=\left\{u \in \mathbb{C}^{\left|\mathcal{C}_{\infty}\right|}:\|u\|_{0}<\infty\right\}
$$


and denote by $\mathcal{P}(\mathcal{X})$ a space of Borel probability measures on $\mathcal{X}$.

Proposition 4.9. Assume that conditions of Proposition 2.7 are fulfilled. Then for any unbounded set $\mathcal{C}_{\infty} \subseteq \mathbb{Z}^{d}$, the corresponding $\mathcal{C}_{\infty}$-effective equation defines a Markov process in $\mathcal{X}$ and is mixing. That is, it has a unique stationary measure $\mu^{\infty}$ in the class of measures $\mathcal{P}(\mathcal{X})$, and for any its solution $u(\tau)$ satisfying $\mathbf{P}(u(\tau) \in \mathcal{X}$ for all $\tau \geq 0)=1$ we have the convergence $\mathcal{D}(u(\tau)) \rightarrow \mu^{\infty}$ as $\tau \rightarrow \infty$.

To prove Proposition 4.9, we will need the following:

Proposition 4.10. Let $u(\tau)$ be a solution of the effective equation (2.37) satisfying $\mathbf{E} \mathrm{e}^{\delta_{0}\|u(0)\|_{0}^{2}}<C$ for some $\delta_{0}>0$. Then, there exists $\delta>0$ such that for any $0<\lambda \leq 1$ and $\tau \geq 0$ we have

$$
\mathbf{E} \max _{s \in[\tau, \tau+1]} \mathrm{e}^{\delta\|u(s)\|_{0}^{2}} \leq C_{1}(\gamma, \delta, C) .
$$

Proof. Note that the norm $\|\cdot\|_{0}$ restricted to $\mathbb{R}^{|\mathcal{C}|}$ coincides with the norm from (2.3) with $j=0$. Let $u=p+i q$. Since $\|u\|_{0}^{2}=\|p\|_{0}^{2}+\|q\|_{0}^{2}$, the upper bound from Proposition 2.2 implies that $\mathbf{E} \mathrm{e}^{\hat{\delta}_{0} U^{0}(u(0))}<C$ for some $\hat{\delta}_{0}>0$, where $U^{0}(u):=U^{0}(p(u), q(u)) .{ }^{26}$ Consider a solution $u^{\varepsilon}(\tau)$ of Eq. (2.25) satisfying $\mathcal{D}\left(u^{\varepsilon}(0)\right)=\mathcal{D}(u(0))$. Due to Lemma 2.3, there exists $\hat{\delta}>0$ such that

$$
\mathbf{E} \max _{s \in[\tau, \tau+1]} \mathrm{e}^{\hat{\delta} U^{0}\left(u^{\varepsilon}(s)\right)}<C_{1},
$$

for any $0<\varepsilon<\varepsilon_{0}, 0<\lambda \leq 1$ and $\tau \geq 0$. Due to the lower bound from Proposition 2.2 , we have $U^{0}(u) \geq\|u\|_{0}^{2} / 4$, so that

$$
\mathbf{E} \max _{s \in[\tau, \tau+1]} \mathrm{e}^{\delta\left\|u^{\varepsilon}(s)\right\|_{0}^{2}} \leq C_{1}
$$

where $\delta:=\hat{\delta} / 4$. Then, Theorem 1.1 joined with the Fatou lemma implies the desired estimate.

Proof of Proposition 4.9. The proof is divided into three steps. In the first one, we show that if $\lambda$ is sufficiently small, then the $\mathcal{C}_{\infty}$-effective equation is contracting in the space $\mathcal{X}$. In the second step, we prove that the $\mathcal{C}_{\infty}$-effective equation is well posed and defines a Markov process in $\mathcal{X}$. In the third one, we show that the $\mathcal{C}_{\infty}$-effective equation has a stationary measure from $\mathcal{P}(\mathcal{X})$, prove that the latter is unique, and obtain the desired convergence.

Step 1. Let $u^{1}(\tau)$ and $u^{2}(\tau)$ be two solutions of the $\mathcal{C}_{\infty}$-effective equation having deterministic initial conditions $u_{0}^{1}, u_{0}^{2} \in \mathcal{X}$ and corresponding to the same Brownian motion $\left(\boldsymbol{\beta}_{j}\right)_{j \in \mathcal{C}_{\infty}}$. Let $w:=u^{1}-u^{2}$. Since the second partial derivatives of the potential $V_{k j}^{\text {res }}$ are bounded, for all $j \in \mathcal{C}_{\infty}$ we have

$$
\frac{\mathrm{d}}{\mathrm{d} \tau} \frac{\left|w_{j}\right|^{2}}{2} \leq \lambda C \sum_{k:|k-j| \leq 1}\left|w_{k}\right|\left|w_{j}\right|-\frac{\left|w_{j}\right|^{2}}{2}, \quad \text { a.s. }
$$

${ }^{26}$ See (2.2) for the definition of $U^{0}$. 
see (2.37). Multiplying the both sides of (4.36) by $\gamma^{|j|}$ and summing over $j \in \mathcal{C}_{\infty}$, we find

$$
\frac{\mathrm{d}}{\mathrm{d} \tau}\|w\|_{0}^{2} \leq(\lambda C-1)\|w\|_{0}^{2}, \quad \text { a.s. }
$$

Applying the Gronwall inequality, we get

$$
\|w(\tau)\|_{0}^{2} \leq\|w(0)\|_{0}^{2} \mathrm{e}^{(\lambda C-1) \tau}, \quad \text { a.s. }
$$

Assume $\lambda<1 / C$. Then

$$
\|w(\tau)\|_{0} \rightarrow 0 \quad \text { as } \tau \rightarrow \infty, \quad \text { a.s. }
$$

Step 2. Let us show first that the $\mathcal{C}_{\infty}$-effective equation admits a weak solution in the space $\mathcal{X}$. Let $u_{0}^{\infty} \in \mathcal{X}$ be a deterministic initial data. Take a sequence of sets $\mathcal{C}_{n}:=\mathcal{C}_{\infty} \cap\left\{j \in \mathbb{Z}^{d}:|j| \leq n\right\}$. Let $u^{\mathcal{C}_{n}}(\tau)$ be a solution of the effective equation (2.37) with $\mathcal{C}=\mathcal{C}_{n}$ and initial conditions $u_{0}^{\mathcal{C}_{n}} \in \mathbb{C}^{\left|\mathcal{C}_{n}\right|}$ satisfying $u_{0 j}^{\mathcal{C}_{n}}=u_{0 j}^{\infty}$ for every $j \in \mathcal{C}_{n}$. We define the process $u^{n}=\left(u_{j}^{n}\right)_{j \in \mathcal{C}_{\infty}}$, where $u_{j}^{n}=u_{j}^{\mathcal{C}_{n}}$, if $j \in \mathcal{C}_{n}$, and $u_{j}^{n}=0$, if $j \in \mathcal{C}_{\infty} \backslash \mathcal{C}_{n}$. Proposition 4.10 joined with the Arzela-Ascoli theorem implies

Proposition 4.11. The set of measures $\left\{\mathcal{D}\left(u^{n}(\cdot)\right), n \in \mathbb{N}\right\}$ is tight in the space $C\left([0, \infty), \mathbb{C}^{\left|\mathcal{C}_{\infty}\right|}\right)$.

Let

$$
\mathcal{D}\left(u^{n_{k}}(\cdot)\right) \rightarrow Q^{\infty} \text { as } k \rightarrow \infty .
$$

Take a process $u^{\infty}(\tau)$ satisfying $\mathcal{D}\left(u^{\infty}(\cdot)\right)=Q^{\infty}$. It is possible to show that $u^{\infty}(\tau)$ is a weak solution of the $\mathcal{C}_{\infty}$-effective equation. Obviously, it satisfies $u^{\infty}(0)=u_{0}^{\infty}$. Moreover, Proposition 4.10 joined with convergence (4.39) and Fatou's lemma implies that $\mathbf{E} \max _{s \in[\tau, \tau+1]} \mathrm{e}^{\delta\left\|u^{\infty}(s)\right\|_{0}^{2}} \leq C$, so

$$
\mathbf{P}\left(u^{\infty}(\tau) \in \mathcal{X} \text { for all } \tau \geq 0\right)=1 .
$$

Estimate (4.37) implies the pathwise uniqueness in $\mathcal{X}$ of solutions for the $\mathcal{C}_{\infty}$-effective equation. Jointly with existence of a weak solution, by YamadaWatanabe arguments (see $[39,46]$ ), the pathwise uniqueness implies existence of a strong solution. Then, using arguments from Chapter 7 of [35], it can be shown that the set of solutions corresponding to all possible initial data from $\mathcal{X}$ forms a Markov family.

Step 3. Let $\mathscr{P}_{\tau}(u, \cdot)$ be the corresponding transition function. Convergence (4.38) implies that for any $u_{0}^{1}, u_{0}^{2} \in \mathcal{X}$ we have

$$
\left\|\mathscr{P}_{\tau}\left(u_{0}^{1}, \cdot\right)-\mathscr{P}_{\tau}\left(u_{0}^{2}, \cdot\right)\right\|_{\text {Lip }}^{*} \rightarrow 0 \quad \text { as } \tau \rightarrow \infty,
$$

where $\|\cdot\|_{\text {Lip }}^{*}$ denotes the dual-Lipschitz norm in $\mathcal{P}(\mathcal{X})$. It follows that, if the $\mathcal{C}_{\infty}$-effective equation has a stationary measure $\mu^{\infty} \in \mathcal{P}(\mathcal{X})$, then it is unique, and for any solution $u(\tau)$ of the $\mathcal{C}_{\infty}$-effective equation satisfying $\mathbf{P}(u(\tau) \in$ $\mathcal{X}$ for all $\tau \geq 0)=1$ we have $\mathcal{D}(u(\tau)) \rightarrow \mu^{\infty}$ (see Step 1 from the proof of Theorem 3.1.3 in [31]). To show that the stationary measure exists, we employ an argument similar to that used in the beginning of Step 2 joined with the estimate of Proposition 2.5.ii. 
Now let us show that Proposition 4.9 implies Proposition 2.7.

Proof of Proposition 2.7. Existence. Estimates of Proposition 2.5 imply that the stationary measure $\mu^{\infty}$ of the $\mathcal{C}_{\infty}$-effective equation constructed in the proof of Proposition 4.9 is regular.

Uniqueness. Let $\mu^{\infty}$ be a regular stationary measure of the $\mathcal{C}_{\infty}$-effective equation. Then, there exists a regular weak solution $u^{\infty}(\tau)$ of the latter satisfying $\mathcal{D}(u(\tau)) \equiv \mu^{\infty}$. Clearly, the regularity implies that $\mathbf{P}\left(u^{\infty}(\tau) \in \mathcal{X}\right.$ for all $\tau \geq$ $0)=1$, so that $\mu^{\infty}$ is a stationary measure of the Markov process in the space $\mathcal{X}$, given by the $\mathcal{C}_{\infty}$-effective equation. Proposition 4.9 provides that such measure is unique.

\section{Acknowledgements}

I am very grateful to my Ph.D. supervisors S. Kuksin and A. Shirikyan for formulation of the problem, guidance and encouragement. I would like to thank N. Cuneo, J.-P. Eckmann, V. Jaksic, J. L. Lebowitz and C. Liverani for useful discussions, and N. Cuneo and J.-P. Eckmann for the excellent stay in Geneva. This work was supported by the Russian Science Foundation under Grant 14-50-00005 and performed in Steklov Mathematical Institute of Russian Academy of Science.

\section{Appendix A. Uniformity of Mixing}

In this appendix, we show that Eq. (1.6) is exponentially mixing with uniform in $\varepsilon$ rate. Note that for fixed $\varepsilon$ the mixing property of Eq. (1.6) is well understood, see e.g. [34], where it is proven for the case of smooth interaction potential $V$. It is convenient to work in the complex variables $u=p+i q \in \mathbb{C}^{|\mathcal{C}|}$, so that instead of (1.6) we deal with Eq. (2.25). The proof is based on the fact that the latter, written in the $a$-variables, has uniformly in $\varepsilon$ bounded coefficients (see Eq. (2.29)).

We do not follow dependence of the rate of mixing on the choice of the set $\mathcal{C}$, so in this section the constants $C, C_{1}, \ldots$ are permitted to depend on $\mathcal{C} .{ }^{27}$ Moreover, for simplicity of notations we put $\lambda=1$. Let $\mathfrak{P}_{\tau}^{\varepsilon}$ be a Markov semigroup associated with Eq. (2.25).

Theorem A.1. Equation (2.25) has a unique stationary measure $\mu^{\varepsilon}$. There exist constants $\varepsilon_{1}>0$ and $C, b>0$ such that for all $0<\varepsilon<\varepsilon_{1}$ and any Borel probability measure $\rho$ satisfying $\left\langle\rho,|u|^{2}\right\rangle<\infty$ we have

$$
\left\|\mathfrak{P}_{\tau *}^{\varepsilon} \rho-\mu^{\varepsilon}\right\|_{\text {var }} \leq C\left(1+\left\langle\rho,|u|^{2}\right\rangle\right) \mathrm{e}^{-b \tau},
$$

where $\|\cdot\|_{\text {var }}$ denotes the variational norm.

${ }^{27}$ We do not know if the rate of mixing is uniform with respect to the choice of the set $\mathcal{C}$. If it is, it seems to be a very complicated problem to prove this. Note that we do not need this uniformity to get in Theorem 2.8 the uniformity of convergence in $\mathcal{C}$. Indeed, in the proof of the latter we do not use the mixing property but we work directly with stationary solutions. 
Proof. In this proof by $B_{R}$, we denote a closed ball in $\mathbb{C}^{|\mathcal{C}|}$ of radius $R$, centred at zero. By $\mathscr{P}_{\tau}^{\varepsilon}(u, \cdot)$, we denote the transition function of the Markov process $(2.25)$.

A standard Bogolioubov-Krylov argument implies existence of a stationary measure $\mu^{\varepsilon}$ for Eq. (2.25). Assume that for any $u_{1}, u_{2} \in \mathbb{C}^{|\mathcal{C}|}, 0<\varepsilon<\varepsilon_{1}$ and $\tau \geq 0$ we have

$$
\left\|\mathscr{P}_{\tau}^{\varepsilon}\left(u_{1}, \cdot\right)-\mathscr{P}_{\tau}^{\varepsilon}\left(u_{2}, \cdot\right)\right\|_{\text {var }} \leq C\left(1+\left|u_{1}\right|^{2}+\left|u_{2}\right|^{2}\right) \mathrm{e}^{-b \tau} .
$$

Using arguments from the introduction to Section 3, and of Sections 3.1.1, 3.1.2 from [31], it can be shown that (A.2) implies the assertion of the theorem. Coupling argument from the introduction to Section 3 of [31] implies that to prove (A.2) it suffices to show that the Markov process given by Eq. (2.25) satisfies the following two properties.

Recurrence. Let $u^{\varepsilon}(\tau)$ be a solution of Eq. (2.25) satisfying $u^{\varepsilon}(0)=u_{1}$, where $u_{1} \in \mathbb{C}^{|\mathcal{C}|}$. Put

$$
\tau_{R}^{\varepsilon}:=\inf \left\{\tau \geq 0: u^{\varepsilon}(\tau) \in B_{R}\right\} .
$$

Then, there exist $R, \delta>0$ and $\varepsilon_{2}>0$ such that for any $0<\varepsilon<\varepsilon_{2}$ we have

$$
\mathbf{E} \mathrm{e}^{\delta \tau_{R}^{\varepsilon}} \leq C\left(1+\left|u_{1}\right|^{2}\right) \text {. }
$$

Squeezing. For any $R>0$, there exist constants $0<\vartheta \leq 1$ and $\varepsilon_{3}>0$ such that for any $u_{1}, u_{2} \in B_{R}$ and $0<\varepsilon<\varepsilon_{3}$ we have

$$
\left\|\mathscr{P}_{1}^{\varepsilon}\left(u_{1}, \cdot\right)-\mathscr{P}_{1}^{\varepsilon}\left(u_{2}, \cdot\right)\right\|_{v a r} \leq 1-\vartheta .
$$

We start with the proof of the recurrence.

Proof of the recurrence. For simplicity of notations, we will skip the upper index $\varepsilon$. Let

$$
U(u)=H(u)+\frac{\varepsilon}{2} \sum_{j \in \mathcal{C}} p_{j} q_{j},
$$

where $u=p+i q, p=\left(p_{j}\right)_{j \in \mathcal{C}}, q=\left(q_{j}\right)_{j \in \mathcal{C}}$. Applying Ito's formula to the function $\mathrm{e}^{\delta \tau} U(u(\tau))$ with $0<\delta<1$, we obtain ${ }^{28}$

$$
\begin{aligned}
\mathrm{e}^{\delta \tau} U(u(\tau))= & U\left(u_{1}\right)+\int_{0}^{\tau} \mathrm{e}^{\delta s}\left(\delta U+\varepsilon^{-1}\{U, H\}-\sum_{j \in \mathcal{C}} p_{j} \partial_{p_{j}} U+\sum_{j \in \mathcal{C}} \mathcal{T}_{j} \partial_{p_{j}^{2}}^{2} U\right) \mathrm{d} s \\
& +\int_{0}^{\tau} \sum_{j \in \mathcal{C}} \mathrm{e}^{\delta s} \sqrt{2 \mathcal{T}_{j}} \partial_{p_{j}} U \mathrm{~d} \beta_{j} .
\end{aligned}
$$

Using estimate (1.20), we get

$$
\begin{aligned}
\varepsilon^{-1}\{U, H\} & =\left\{\frac{1}{2} \sum_{j \in \mathcal{C}} p_{j} q_{j}, \sum_{k \in \mathcal{C}} \frac{p_{k}^{2}+q_{k}^{2}}{2}\right\}+\left\{\frac{1}{2} \sum_{j \in \mathcal{C}} p_{j} q_{j}, \frac{\varepsilon}{2} \sum_{|k-m|=1} V\left(q_{m}, q_{k}\right)\right\} \\
& \leq \frac{1}{2}\left(|p|^{2}-|q|^{2}\right)+C_{1} \varepsilon\left(1+|q|^{2}\right) .
\end{aligned}
$$

\footnotetext{
28 Here, it is simpler to look at Eq. (1.6) than at (2.25).
} 
Moreover, for $\varepsilon$ sufficiently small,

$$
\begin{gathered}
|u|^{2} / 4 \leq U(u) \leq C_{2}\left(1+|u|^{2}\right), \quad-\sum_{j \in \mathcal{C}} p_{j} \partial_{p_{j}} U \leq(-1+\varepsilon)|p|^{2}+\varepsilon|q|^{2}, \\
\sum_{j \in \mathcal{C}} \mathcal{T}_{j} \partial_{p_{j}^{2}}^{2} U=\sum_{j \in \mathcal{C}} \mathcal{T}_{j} \leq C_{3} .
\end{gathered}
$$

For $K>0$ put $\xi:=\tau_{R}^{\varepsilon} \wedge K$. Then, (A.5) joined with (A.6) and (A.7) implies

$$
\mathbf{E} \mathrm{e}^{\delta \xi} U(u(\xi)) \leq U\left(u_{1}\right)+\mathbf{E} \int_{0}^{\xi} \mathrm{e}^{\delta s}\left(\Delta|u|^{2}+C_{4}\right) \mathrm{d} s,
$$

where

$$
\begin{aligned}
\Delta & :=\left[\left(\delta C_{2}+1 / 2-1+\varepsilon\right) \vee\left(\delta C_{2}-1 / 2+C_{1} \varepsilon+\varepsilon\right)\right] \\
& =\left[\left(-1 / 2+\delta C_{2}+\varepsilon\right) \vee\left(-1 / 2+\delta C_{2}+\varepsilon\left(C_{1}+1\right)\right)\right],
\end{aligned}
$$

and $C_{4}:=C_{2}+C_{1}+C_{3}$. Choose $\varepsilon_{2}$ and $\delta$ in such a way that for $\varepsilon \leq \varepsilon_{2}$ we have $\Delta(\varepsilon, \delta)<0$, and fix $R \geq \sqrt{-C_{4} / \Delta\left(\varepsilon_{2}, \delta\right)}$. Let $s \leq \xi$. Then, $|u(s)| \geq R$, so that $\Delta|u(s)|^{2}+C_{4} \leq 0$, for any $\varepsilon \leq \varepsilon_{2}$. Thus, (A.8) implies

$$
\mathbf{E} \mathrm{e}^{\delta \xi} U(u(\xi)) \leq U\left(u_{1}\right) \text {. }
$$

Since, in view of the first estimate from (A.7), we have $U(u(\xi)) \geq|u(\xi)|^{2} / 4 \geq$ $R^{2} / 4$, we obtain

$$
\mathbf{E} \mathrm{e}^{\delta \xi} \leq 4 U\left(u_{1}\right) / R^{2} \leq 4 C_{2}\left(1+\left|u_{1}\right|^{2}\right) / R^{2} .
$$

Letting $K \rightarrow \infty$ and applying Fatou's lemma, we get (A.3).

Proof of the squeezing. Denote by $\mathscr{P}^{a, \varepsilon}(s, \tau ; u, \cdot)$ the transition function of the Markov process (2.29), corresponding to initial conditions $u$ and initial time $s$. Let $\mathscr{P}_{\tau}^{a, \varepsilon}(u, \cdot):=\mathscr{P}^{a, \varepsilon}(0, \tau ; u, \cdot)$. In view of the definition of the $a$-variables (2.27), we obviously have

$$
\left\|\mathscr{P}_{1}^{\varepsilon}\left(u_{1}, \cdot\right)-\mathscr{P}_{1}^{\varepsilon}\left(u_{2}, \cdot\right)\right\|_{\text {var }}=\left\|\mathscr{P}_{1}^{a, \varepsilon}\left(u_{1}, \cdot\right)-\mathscr{P}_{1}^{a, \varepsilon}\left(u_{2}, \cdot\right)\right\|_{\text {var }} .
$$

Let $\mathcal{G} \in \mathcal{B}\left(\mathbb{C}^{|\mathcal{C}|}\right)$. First, we will obtain uniform in $\varepsilon$ and $u \in B_{R}$ estimates from below for the transition probability $\mathscr{P}_{1}^{a, \varepsilon}(u, \mathcal{G})$. For this purpose, we will use Girsanov's theorem (see [35]). Let $a^{\varepsilon}(\tau)$ be a solution of Eq. (2.29) satisfying $a^{\varepsilon}(0)=u$, where $u \in B_{R}$, and

$$
\xi_{K}^{\varepsilon}=\inf \left\{\tau \geq 0:\left|a^{\varepsilon}(\tau)\right| \geq K\right\}
$$

To satisfy Novikov's condition needed for application of Girsanov's theorem (see below), instead of considering the process $a^{\varepsilon}(\tau)$ we will consider the process $a^{\varepsilon, K}(\tau)$ which coincides with $a^{\varepsilon}(\tau)$ for $\tau \leq \xi_{K}^{\varepsilon}$ and for $\tau \geq \xi_{K}^{\varepsilon}$ satisfies

$$
\dot{a}_{j}^{\varepsilon, K}=\sqrt{2 \mathcal{T}_{j}} \mathrm{e}^{-i \varepsilon^{-1} \tau} \dot{\beta}_{j}, \quad j \in \mathcal{C} .
$$

Writing Eq. (2.29) in the real coordinates and using that $P_{j}$ are real valued functions, we see that Girsanov's theorem implies that for all $0 \leq \tau \leq 1$ the process $a^{\varepsilon, K}(\tau)$ satisfies the equation

$$
\dot{a}_{j}^{\varepsilon, K}=\sqrt{2 \mathcal{T}_{j}} \mathrm{e}^{-i \varepsilon^{-1} \tau} \dot{\beta}_{j}^{\varepsilon, K}, \quad a_{j}^{\varepsilon, K}(0)=u_{j}, \quad j \in \mathcal{C},
$$


where $\left(\beta_{j}^{\varepsilon, K}\right)_{j \in \mathcal{C}}$ is a standard $|\mathcal{C}|$-dimensional real Brownian motion with respect to the measure $\mathbf{Q}^{\varepsilon, K}$ given by

$$
d \mathbf{Q}^{\varepsilon, K}(\omega)=M^{\varepsilon, K}(\omega) d \mathbf{P}(\omega)
$$

with

$$
M^{\varepsilon, K}=\exp \left(-\int_{0}^{1 \wedge \xi_{K}^{\varepsilon}} \sum_{j \in \mathcal{C}} \mathcal{P}_{j}^{\varepsilon}\left(\tau, a^{\varepsilon, K}(\tau)\right) \mathrm{d} \beta_{j}-\frac{1}{2} \int_{0}^{1 \wedge \xi_{K}^{\varepsilon}}\left|\mathcal{P}^{\varepsilon}\left(\tau, a^{\varepsilon, K}(\tau)\right)\right|^{2} \mathrm{~d} \tau\right),
$$

where by $\mathcal{P}^{\varepsilon}=\left(\mathcal{P}_{j}^{\varepsilon}\right)_{j \in \mathcal{C}}$ we have denoted

$$
\mathcal{P}_{j}^{\varepsilon}(\tau, u):=\left(2 \mathcal{T}_{j}\right)^{-1 / 2} P_{j}\left(\mathrm{e}^{i \varepsilon^{-1} \tau} u\right)
$$

Note that Novikov's condition $\mathbf{E} \exp \left(\frac{1}{2} \int_{0}^{1 \wedge \xi_{K}^{\varepsilon}}\left|\mathcal{P}^{\varepsilon}\left(\tau, a^{\varepsilon, K}(\tau)\right)\right|^{2} \mathrm{~d} \tau\right)<\infty$, required for the application of Girsanov's theorem, is satisfied since for $\tau \leq \xi_{K}^{\varepsilon}$ we have $\left|a^{\varepsilon, K}(\tau)\right| \leq K$.

Now let us estimate the transition probability $\mathscr{P}_{1}^{a, \varepsilon}(u, \mathcal{G})=$ $\mathbf{P}\left(a^{\varepsilon}(1) \in \mathcal{G}\right)$. We have

$$
\begin{aligned}
& \mathbf{P}\left(a^{\varepsilon}(1) \in \mathcal{G}\right) \geq \mathbf{P}\left(a^{\varepsilon}(1) \in \mathcal{G}, \max _{0 \leq \tau \leq 1}\left|a^{\varepsilon}(\tau)\right|<K\right) \\
& \quad=\mathbf{P}\left(a^{\varepsilon, K}(1) \in \mathcal{G}, \max _{0 \leq \tau \leq 1}\left|a^{\varepsilon}(\tau)\right|<K\right) \geq \mathbf{P}\left(a^{\varepsilon, K}(1) \in \mathcal{G}\right)-\rightsquigarrow(K),
\end{aligned}
$$

where Lemma 2.1.i joined with (2.28) implies that $æ(K) \rightarrow 0$ as $K \rightarrow \infty$ uniformly in $\varepsilon$ sufficiently small. Due to (A.11), for any $L>0$, we have

$$
\begin{aligned}
\mathbf{P}\left(a^{\varepsilon, K}(1) \in \mathcal{G}\right) & =\int_{\left\{a^{\varepsilon, K}(1) \in \mathcal{G}\right\}}\left(M^{\varepsilon, K}\right)^{-1} d \mathbf{Q}^{\varepsilon, K} \\
& \geq \mathrm{e}^{-L} \mathbf{Q}^{\varepsilon, K}\left(a^{\varepsilon, K}(1) \in \mathcal{G}, M^{\varepsilon, K} \leq \mathrm{e}^{L}\right) \\
& \geq \mathrm{e}^{-L}\left(\mathbf{Q}^{\varepsilon, K}\left(a^{\varepsilon, K}(1) \in \mathcal{G}\right)-\mathrm{e}^{-L}\right),
\end{aligned}
$$

where we have employed the exponential supermartingale inequality. In view of (A.10), the random variable $a^{\varepsilon, K}(1)$ has the Gaussian distribution with mean $u$, with respect to the measure $\mathbf{Q}^{\varepsilon, K}$. Its covariance matrix written with respect to the real coordinates has the form $D=\operatorname{diag}\left(\begin{array}{cc}D_{j}^{11} & D_{j}^{12} \\ D_{j}^{12} & D_{j}^{22}\end{array}\right)_{j \in \mathcal{C}}$, where

$$
\begin{aligned}
& D_{j}^{11}=2 \mathcal{T}_{j} \int_{0}^{1} \cos ^{2}\left(\varepsilon^{-1} \tau\right) \mathrm{d} \tau=\mathcal{T}_{j}\left(1+\frac{\varepsilon}{2} \sin \left(2 \varepsilon^{-1}\right)\right), \\
& D_{j}^{22}=2 \mathcal{T}_{j} \int_{0}^{1} \sin ^{2}\left(\varepsilon^{-1} \tau\right) \mathrm{d} \tau=\mathcal{T}_{j}\left(1-\frac{\varepsilon}{2} \sin \left(2 \varepsilon^{-1}\right)\right),
\end{aligned}
$$




$$
D_{j}^{12}=2 \mathcal{T}_{j} \int_{0}^{1} \sin \left(\varepsilon^{-1} \tau\right) \cos \left(\varepsilon^{-1} \tau\right) \mathrm{d} \tau=\frac{\varepsilon \mathcal{T}_{j}}{2}\left(1-\cos \left(2 \varepsilon^{-1}\right)\right) .
$$

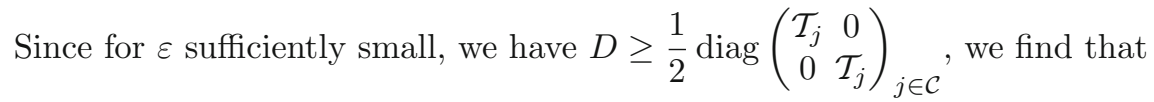

$$
\mathbf{Q}^{\varepsilon, K}\left(a^{\varepsilon, K}(1) \in \mathcal{G}\right) \geq \mathbf{Q}^{\varepsilon, K}\left(a^{\varepsilon, K}(1) \in \mathcal{G} \cap B_{1}\right) \geq C(u) \operatorname{Leb}\left(\mathcal{G} \cap B_{1}\right),
$$

where $\operatorname{Leb}\left(\mathcal{G} \cap B_{1}\right)$ denotes the Lebesgue measure of the set $\mathcal{G} \cap B_{1}$. Obviously,

$$
\hat{C}:=\min _{u \in B_{R}} C(u)>0 .
$$

Combining estimates (A.12)-(A.15), for any $u \in B_{R}$ we get

$$
\mathscr{P}_{1}^{a, \varepsilon}(u, \mathcal{G}) \geq \mathrm{e}^{-L}\left(\hat{C} \operatorname{Leb}\left(\mathcal{G} \cap B_{1}\right)-\mathrm{e}^{-L}\right)-x(K) .
$$

Now we are able to estimate the variational norm (A.9). Without loss of generality, we assume $\mathscr{P}_{1}^{a, \varepsilon}\left(u_{1}, \mathcal{G}\right) \geq \mathscr{P}_{1}^{a, \varepsilon}\left(u_{2}, \mathcal{G}\right)$. Then, in view of (A.16),

$$
\begin{aligned}
\left|\mathscr{P}_{1}^{a, \varepsilon}\left(u_{1}, \mathcal{G}\right)-\mathscr{P}_{1}^{a, \varepsilon}\left(u_{2}, \mathcal{G}\right)\right| & =1-\mathscr{P}_{1}^{a, \varepsilon}\left(u_{1}, \overline{\mathcal{G}}\right)-\mathscr{P}_{1}^{a, \varepsilon}\left(u_{2}, \mathcal{G}\right) \\
& \leq 1-\mathrm{e}^{-L}\left(\hat{C} \operatorname{Leb}\left(B_{1}\right)-2 \mathrm{e}^{-L}\right)+2 æ(K) .
\end{aligned}
$$

Choosing first $L$ and then $K$ sufficiently large, we obtain

$$
\left|\mathscr{P}_{1}^{a, \varepsilon}\left(u_{1}, \mathcal{G}\right)-\mathscr{P}_{1}^{a, \varepsilon}\left(u_{2}, \mathcal{G}\right)\right| \leq 1-\vartheta,
$$

where $\vartheta:=\mathrm{e}^{-L}\left(\hat{C} \operatorname{Leb}\left(B_{1}\right)-2 \mathrm{e}^{-L}\right)-2 æ(K)>0$. It remains to note that the constant $\vartheta$ is independent from $\varepsilon, u_{1}, u_{2} \in B_{R}$ and $\mathcal{G} \in \mathcal{B}\left(\mathbb{C}^{|\mathcal{C}|}\right)$. Then, in view of (A.9), we arrive at (A.4).

\section{Appendix B. Low Temperature Regime}

Let $(\widetilde{p}, \widetilde{q})=\left(\widetilde{p}_{j}, \widetilde{q}_{j}\right)_{j \in \mathcal{C}} \in \mathbb{R}^{2|\mathcal{C}|}$. Consider the equation

$$
\frac{\mathrm{d}}{\mathrm{d} t} \widetilde{q}_{j}=\widetilde{p}_{j}, \quad \frac{\mathrm{d}}{\mathrm{d} t} \widetilde{p}_{j}=-\widetilde{q}_{j}-\varepsilon \sum_{k:|k-j|=1} \partial_{\widetilde{q}_{j}} V\left(\widetilde{q}_{j}, \widetilde{q}_{k}\right)-\varepsilon \widetilde{p}_{j}+\sqrt{2 \varepsilon \delta \mathcal{\mathcal { T } _ { j }}} \frac{\mathrm{d}}{\mathrm{d} t} \beta_{j}, j \in \mathcal{C},
$$

where $\varepsilon, \delta \ll 1$. It describes a system of weakly coupled oscillators, weakly interacting with thermostats of temperatures $\delta \mathcal{T}_{j}, j \in \mathcal{C}$. Let us put

$$
p_{j}:=\sqrt{\delta} \widetilde{p}_{j}, \quad q_{j}:=\sqrt{\delta} \widetilde{q}_{j},
$$

and assume that the interaction potential $V$ is a homogeneous polynom of degree $m \geq 3$. Then

$$
\begin{aligned}
& \frac{\mathrm{d}}{\mathrm{d} t} q_{j}=p_{j}, \\
& \frac{\mathrm{d}}{\mathrm{d} t} p_{j}=-q_{j}-\varepsilon \delta^{(m-2) / 2} \sum_{k:|k-j|=1} \partial_{q_{j}} V\left(q_{j}, q_{k}\right)-\varepsilon p_{j}+\sqrt{2 \varepsilon \mathcal{\mathcal { T } _ { j }}} \frac{\mathrm{d}}{\mathrm{d} t} \beta_{j}, \quad j \in \mathcal{C} .
\end{aligned}
$$

Putting $\lambda:=\delta^{(m-2) / 2}$, we arrive at Eq. (1.6), written in the fast time $t$.

At the physical level of rigor, the condition above for the form of the interaction potential can be weaken. Since we study small amplitude solutions, 
the interaction potential $V\left(\widetilde{q}_{j}, \widetilde{q}_{k}\right)$ can be replaced by a leading order term of its Taylor series. Thus, it suffices to assume that the latter is a homogeneous polynom of degree $m \geq 3$.

\section{Appendix C. Resonant Averaging}

Here, we discuss some properties of the resonant averaging, given by formulas (2.33) and (2.34). Since we will use the derivatives with respect to the angles $\varphi=\left(\varphi_{j}\right)_{j \in \mathcal{C}}$, let us note that if a function $f: \mathbb{C}^{|\mathcal{C}|} \mapsto \mathbb{R}$ is $C^{1}$-smooth, then $f$ is continuously differentiable with respect to the angles $\varphi$. Indeed, this follows from the formula:

$$
\partial_{\varphi_{j}} f=i u_{j} \partial_{u_{j}} f-i \bar{u}_{j} \partial_{\bar{u}_{j}} f .
$$

Proposition C.1. Let $f: \mathbb{C}^{|\mathcal{C}|} \rightarrow \mathbb{R}$ be a continuous function. Then

(i) For any $\xi \in[0,2 \pi)$ we have $\langle f\rangle_{R}\left(\mathrm{e}^{i \xi} u\right) \equiv\langle f\rangle_{R}(u)$.

(ii) Let $f \in \mathcal{L}_{b}\left(\mathbb{C}^{|\mathcal{C}|}\right), \mathcal{L}_{\text {loc }}\left(\mathbb{C}^{|\mathcal{C}|}\right)$ or $C^{n}\left(\mathbb{C}^{|\mathcal{C}|}\right)$, where $n \in \mathbb{N}$. Then, $\langle f\rangle_{R} \in$ $\mathcal{L}_{b}\left(\mathbb{C}^{|\mathcal{C}|}\right), \mathcal{L}_{\text {loc }}\left(\mathbb{C}^{|\mathcal{C}|}\right)$ or $C^{n}\left(\mathbb{C}^{|\mathcal{C}|}\right)$ correspondingly. If partial derivatives of the function $f$ have at a most polynomial growth at infinity, then those of $\langle f\rangle_{R}$ also have at most the polynomial growth.

(iii) Let $f \in C^{1}\left(\mathbb{C}^{|\mathcal{C}|}\right)$. Then for every $j \in \mathcal{C}$ we have $\left\langle\partial_{\varphi_{j}} f\right\rangle_{R} \equiv \partial_{\varphi_{j}}\langle f\rangle_{R}$.

(iv) Let $f \in C^{1}\left(\mathbb{C}^{|\mathcal{C}|}\right)$ be such that $\operatorname{Supp} f \subseteq\{j, k\}$. Then, $\partial_{\varphi_{j}}\langle f\rangle_{R}=-\partial_{\varphi_{k}}\langle f\rangle_{R}$.

Proof. Items (i) and (ii) follow from formula (2.34). Item (iii) follows from (2.33). To prove item (iv), it suffices to note that, in view of (2.33), the resonant averaging $\langle f\rangle_{R}(I, \varphi)$ depends on the angles $\varphi_{j}, \varphi_{k}$ only through their difference $\varphi_{j}-\varphi_{k}$.

\section{References}

[1] Arnold, V., Kozlov, V.V., Neistadt, A.I.: Mathematical Aspects of Classical and Celestial Mechanics, 3rd edn. Springer, Berlin (2006)

[2] Basile, G., Bernardin, C., Olla, S.: Thermal conductivity for a momentum conservative model. Commun. Math. Phys. 287, 67-98 (2009)

[3] Basile, G., Olla, S., Spohn, H.: Energy transport in stochastically perturbed lattice dynamics. Arch. Rat. Mech. Anal. 195, 171-203 (2010)

[4] Bernardin, C., Huveneers, F.: Small perturbation of a disordered harmonic chain by a noise and an anharmonic potential. Probab. Theory Relat. Fields 157, 301331 (2013)

[5] Bernardin, C., Huveneers, F., Lebowitz, J.L., Liverani, C., Olla, S.: GreenKubo formula for weakly coupled system with dynamical noise. Commun. Math. Phys. 334, 1377-1412 (2015)

[6] Bernardin, C., Kannan, V., Lebowitz, J.L., Lukkarinen, J.: Harmonic systems with bulk noises. J. Stat. Phys. 146, 800-831 (2012)

[7] Bernardin, C., Olla, S.: Fourier's law for a microscopic model of heat conduction. J. Stat. Phys. 118, 271-289 (2005) 
[8] Bernardin, C., Olla, S.: Transport properties of a chain of anharmonic oscillators with random flip of velocities. J. Stat. Phys. 145, 1224-1255 (2011)

[9] Bogachev, V.I., Krylov, N.V., Rockner, M.: On regularity of transition probabilities and invariant measures of singular diffusions under minimal conditions. Commun. Partial Differ. Equ. 26, 2037-2080 (2001)

[10] Bonetto, F., Lebowitz, J.L., Lukkarinen, J.: Fourier's law for a harmonic crystal with self-consistent stochastic reservoirs. J. Stat. Phys. 116, 783-813 (2004)

[11] Bonetto, F., Lebowitz, J.L., Lukkarinen, J., Olla, S.: Heat conduction and entropy production in anharmonic crystals with self-consistent stochastic reservoirs. J. Stat. Phys. 134, 1097-1119 (2009)

[12] Bonetto, F., Lebowitz, J.L., Rey-Bellet, L.: Fourier's Law: A Challenge to Theorists. Mathematical Physics 2000, pp. 128-150. Imp. Coll. Press, London (2000)

[13] Carmona, P.: Existence and uniqueness of an invariant measure for a chain of oscillators in contact with two heat baths. Stoch. Process. Appl. 117, 10761092 (2007)

[14] Cuneo, N., Eckmann, J.-P.: Non-equilibrium steady states for chains of four rotors. (2015). arXiv:1504.04964

[15] Cuneo, N., Eckmann, J.-P., Poquet, C.: Non-equilibrium steady state and subgeometric ergodicity for a chain of three coupled rotors. Nonlinearity 28, 2397$2421(2015)$

[16] Dolgopyat, D., Liverani, C.: Energy transfer in a fast-slow Hamiltonian system. Commun. Math. Phys. 308, 201-225 (2011)

[17] Dudley, R.M.: Real Analysis and Probability. Cambridge University Press, Cambridge (2002)

[18] Dymov, A.V.: Dissipative effects in a linear Lagrangian system with infinitely many degrees of freedom. Izv. Math. 76, 1116-1149 (2012)

[19] Dymov, A.: Nonequilibrium statistical mechanics of Hamiltonian rotators with alternated spins. J. Stat. Phys. 158, 968-1006 (2015)

[20] Eckmann, J.-P., Hairer, M.: Non-equilibrium statistical mechanics of strongly anharmonic chains of oscillators. Commun. Math. Phys. 212, 105-164 (2000)

[21] Eckmann, J.-P., Pillet, C.-A., Rey-Bellet, L.: Non-equilibrium statistical mechanics of anharmonic chains coupled to two heat baths at different temperatures. Commun. Math. Phys. 201, 657-697 (1999)

[22] Freidlin, M.I., Wentzell, A.D.: Long-time behavior of weakly coupled oscillators. J. Stat. Phys. 123, 1311-1337 (2006)

[23] Freidlin, M., Wentzell, A.: Random Perturbations of Dynamical Systems, 3rd edn. Springer, Berlin, Heidelberg (2012)

[24] Hairer, M., Mattingly, J.C.: Slow energy dissipation in anharmonic oscillator chains. Commun. Pure Appl. Math. 62, 999-1032 (2009)

[25] Karatzas, I., Shreve, S.: Brownian Motion and Stochastic Calculus, 2nd edn. Springer, Berlin (1991)

[26] Khasminskii, R.: Stochastic Stability of Differential Equations, 2nd edn. Springer, Berlin (2012)

[27] Kuksin, S.B.: Damped-driven KdV and effective equations for long-time behaviour of its solutions. GAFA 20, 1431-1463 (2010) 
[28] Kuksin, S.B.: Weakly nonlinear stochastic CGL equations. Ann. IHP PR 49, 1033-1056 (2013)

[29] Kuksin, S., Maiocchi, A.: Resonant averaging for weakly nonlinear stochastic Schrodinger equations. (2013). arXiv:1309.5022

[30] Kuksin, S.B., Piatnitski, A.L.: Khasminskii-Witham averaging for randomly perturbed KdV equation. J. Math. Pures Appl. 89, 400-428 (2008)

[31] Kuksin, S., Shirikyan, A.: Mathematics of Two-Dimensional Turbulence. Cambridge University Press, Cambridge (2012)

[32] Lepri, S., Livi, R., Politi, A.: Thermal conduction in classical low-dimensional lattices. Phys. Rep. 377, 1-80 (2003)

[33] Liverani, C., Olla, S.: Toward the Fourier law for a weakly interacting anharmonic crystal. AMS 25, 555-583 (2012)

[34] Mattingly, J.C., Stuart, A.M., Higham, D.J.: Ergodicity for SDEs and approximations: locally Lipschitz vector fields and degenerate noise. Stoch. Process. Appl. 101, 185-232 (2002)

[35] Øksendal, B.: Stochastic Differential Equations. Springer, Berlin (2003)

[36] Peierls, R.: On the Kinetic Theory of Thermal Conduction in Crystals. Selected Scientific Papers of Sir Rudolf Peierls, with Commentary, pp. 15-48. World Scientific, Singapore (1997)

[37] Pardoux, E., Veretennikov, A.Yu.: On the Poisson equation and diffusion approximation I. Ann. Probab. 29, 1061-1085 (2001)

[38] Rey-Bellet, L., Thomas, L.E.: Exponential convergence to non-equilibrium stationary states in classical statistical mechanics. Commun. Math. Phys. 225, 305329 (2002)

[39] Rockner, M., Schmuland, B., Zhang, X.: Yamada-Watanabe theorem for stochastic evolution equations in infinite dimensions. Condens. Matter Phys. 11, 247-259 (2008)

[40] Ruelle, D.A.: Mechanical model for Fourier's law of heat conduction. Commun. Math. Phys. 311, 755-768 (2012)

[41] Spohn, H.: Large Scale Dynamics of Interacting Particles. Springer, Heidelberg (1991)

[42] Taylor, M.E.: Partial Differential Equations I: Basic Theory. Springer, New York (1996)

[43] Treschev, D.: Oscillator and thermostat. Discrete Contin. Dyn. Syst. 28, 1693$1712(2010)$

[44] Veretennikov, A.: Bounds for the mixing rate in the theory of stochastic equations. Theory Probab. Appl. 32, 273-281 (1987)

[45] Veretennikov, A.Yu.: On polynomial mixing bounds for stochastic differential equations. Stoch. Process. Appl. 70, 115-127 (1997)

[46] Yor, M.: Existence et unicité de diffusion à valeurs dans un espace de Hilbert. Ann. IHP B 10, 55-88 (1974) 
Andrey Dymov

Mathematics Department

Université de Cergy-Pontoise

CNRS 95000 Cergy-Pontoise, France

Andrey Dymov

Steklov Mathematical Institute

Moscow, Russia

e-mail: adymov88@gmail.com

Communicated by Christian Maes.

Received: March 12, 2015.

Accepted: September 1, 2015. 